\title{
PIV and Rotational Raman-Based Temperature Measurements for CFD Validation of a Perforated Plate Cooling Flow: Part I
}

\author{
Mark P. Wernet ${ }^{*}$ and Nicholas J. Georgiadis* \\ NASA Glenn Research Center, Cleveland, OH, 44135 \\ Randy J. Locke \\ Vantage Partners LLC, Cleveland, OH, 44135 \\ Douglas R. Thurman \\ Army Research Lab., Cleveland, $\mathrm{OH}, 44135$ \\ Philip E. Poinsatte \\ NASA Glenn Research Center, Cleveland, OH, 44135
}

\begin{abstract}
Film cooling is used in a wide variety of engineering applications for protection of surfaces from hot or combusting gases. The design of more efficient film cooling geometries/configurations could be facilitated by an ability to accurately model and predict the effectiveness of current designs using computational fluid dynamics (CFD) code predictions. Hence, a benchmark set of flow field property data was obtained to assess current CFD capabilities and develop better modeling approaches for these turbulent flow fields where accurate calculation of turbulent heat flux is important. Both Particle Image Velocimetry (PIV) and spontaneous rotational Raman scattering (SRS) spectroscopy were used to acquire high quality, spatially-resolved measurements of the mean and root mean square (rms) velocities as well as the mean and rms temperatures in a film cooling flow field. In addition to off-body flow field measurements, infrared thermography (IR) and thermocouple measurements on the plate surface enabled estimates of the film effectiveness. Raman spectra in air were obtained across a matrix of axial locations downstream from a $68.07 \mathrm{~mm}$ square nozzle blowing heated air over a range of temperatures (up to TR $=2.7$ ) and Mach numbers (up to Mach 0.9), across a $30.48 \mathrm{~cm}$ long plate equipped with three patches of 45 small $(\sim 1 \mathrm{~mm})$ diameter cooling holes arranged in a staggered configuration. In addition, both streamwise 2-component PIV (on the plate centerline) and cross-stream 3-component Stereo PIV data at 14 axial stations were collected in the same flows. Only a subset of the data collected in the test program is included in this Part I report. The rest of the data will be published in a future report, Part II, along with planned CFD predictions of the complex cooling film flow.

The entire data set of Raman temperature data, PIV velocity data and IR camera data covering the Set Points 23 and 49 in the test matrix in Table 1 is available in an accompanying DVD (available online from www.sti.nasa.gov) for those interested in further analysis.
\end{abstract}

\section{Acronyms}

\section{Nomenclature}

AAPL AeroAcoustic Propulsion Lab

BOS Background Oriented Schlieren

BR Blowing ratio based on total mass flow rates and areas $=\left(m_{\text {inj }} / m_{j}\right) \cdot\left(A_{j} / A_{i n j}\right) \sim(\rho U)_{\text {inj }} /(\rho U)_{j}$

CFD Computational Fluid Dynamics

GRC Glenn Research Center

NPR Ratio of the stagnation pressure of the jet to the ambient pressure

PIV Particle Image Velocimetry

rms Root Mean Square

SHJAR Small Hot Jet Acoustic Rig

SRS Spontaneous Raman Scattering

TR Temperature ratio: $T_{j} / T_{\infty}$

Symbols

$\mathrm{A}_{\text {inj }} \quad$ Total injector tube area $(135$ holes $)=109.4 \mathrm{~mm}^{2}$

$A_{j} \quad$ Nozzle area at jet exit $=4634 \mathrm{~mm}^{2}$

$\mathrm{D} \quad$ Injector hole diameter $=1.016 \mathrm{~mm}$

$m_{\text {inj }} \quad$ Mass flow rate of the air supplied through the cooling holes $[\mathrm{kg} / \mathrm{s}]$

$\mathrm{m}_{\mathrm{j}} \quad$ Mass flow rate of the heated air from the square nozzle $[\mathrm{kg} / \mathrm{s}]$

M Mach number

\footnotetext{
${ }^{*}$ AIAA Associate Fellow
} 


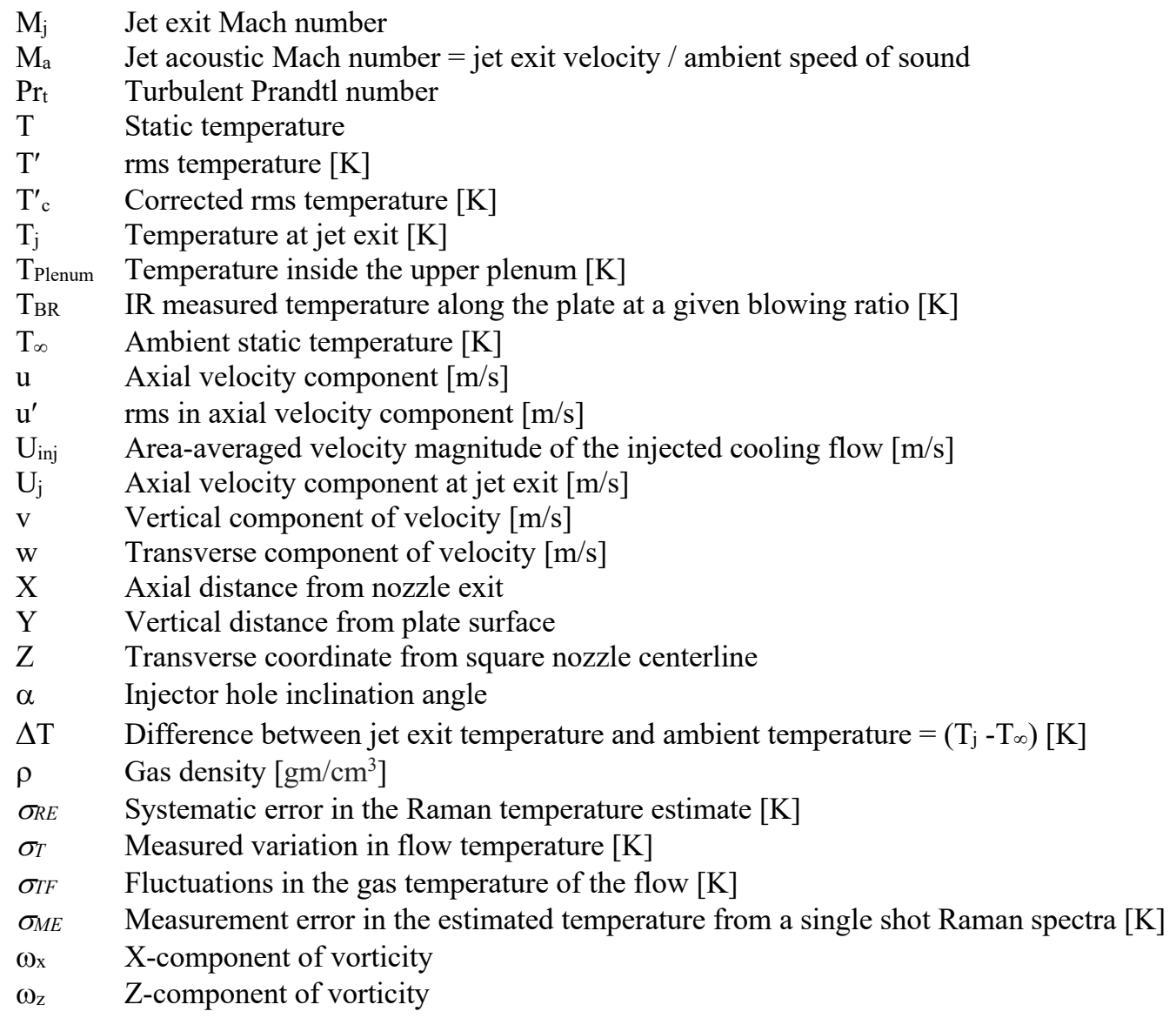

\subsection{Introduction}

The performance of advanced gas turbine engines is limited by cycle temperatures within the engine. Cooling the internal engine surfaces is necessary to maintain the metal surface temperatures within their material property limits. Thin film cooling is predominantly used in gas turbine engines for cooling turbine blades, combustion chamber walls and the liners in afterburning engines [Bogard \& Thole 2005]. There are also situations where hot flow from an engine exhaust may flow over a portion of the vehicle, as in aircraft that employ integrated propulsion systems. These surfaces also frequently require cooling for the structure to survive. Hence, a better understanding of the aerothermodynamics and an ability to accurately predict the performance of thin cooling films using computational analysis tools is required in order to improve the durability and performance of aircraft engine and other aerospace vehicle components.

Many thin film cooling studies have employed single hole cooling configurations in order to optimize the single cooling hole efficiency. Smith and Mungal, (1998) characterized cooling plume trajectories using acetone Planar Laser Induced Fluorescence (PLIF) and determined that the desired feature of film cooling jets is that they are re-entrained into the boundary layer far downstream. The mean properties of the boundary layer recover to their initial state, as if the jet never existed. This distinguishes them from jets for other industrial applications with much higher blowing ratios (BRs). Low BR's are desirable in order to avoid generation of a counter rotating vortex pair, which has a sense of rotation that can promote jet lift-off and cause entrainment of heated main flow towards the wall, and in turn decreases the desired film cooling. Thus, a primary consideration in improving film cooling performance is to avoid jet lift off and minimize the entrainment of high temperature main stream fluid near the wall.

The design variables having the most impact on the film cooling performance are the inclination angle of the cooling jets, the $B R$ and the spatial separation of the jets. In many of the fundamental single jet studies, $B R=(\rho U)_{\text {inj }} /(\rho U)_{j}$ is defined by the quantities in the single jet core. In the experiments to be described in this paper, with multiple small holes, these quantities are defined by an area-average, or $\mathrm{BR}=\left(\mathrm{m}_{\mathrm{inj}} / \mathrm{m}_{\mathrm{j}}\right)\left(\mathrm{A}_{\mathrm{j}} / \mathrm{A}_{\mathrm{inj}}\right)$.

It is generally accepted that inclined jets exhibit less penetration and less spreading in the vertical and lateral directions. This indicates that shallower angles provide less lateral mixing than steeper injection holes. Bergeles et al., (1977) performed pitot probe measurements of the flow field formed by a jet inclined $\alpha=30^{\circ}$ to a flat plate. The maximum effectiveness and coverage occurred at a blowing ratio $\mathrm{BR} \approx 0.5$, after which the jet began to lift-off the wall. Baldauf et 
al., (2001) used IR cameras to estimate adiabatic film cooling effectiveness over a range of inclination angles, hole pitch and blowing ratios, where again the $30^{\circ}$ inclination angle and $\mathrm{BR} \approx 0.5$ were determined to be optimal. Wernet et al., (2018) were the first to use Particle Imaging Velocimetry (PIV), Rotationally Resolved Raman scattering, and embedded thermocouples to characterize both the flow field velocity and gas temperature of a single jet issuing into a heated freestream flow above a flat plate at elevated velocities (Mach 0.3 to Mach 0.9) and temperatures (up to 950K) over a range of blowing ratios. The PIV and Raman temperature measurements clearly depicted the lift-off of the injected plume and defined the mixing layer between the hot freestream flow and the injected film cooling flow. The velocity and temperature measurements were compared to Reynolds-averaged Navier-Stokes (RANS) CFD predictions of the flow, demonstrating that RANS underpredicts the mixing of the jet with the main stream flow. This PIV/Raman data set was collected to aid in the development of more comprehensive flow turbulence models to more accurately predict these complex flows.

While single hole studies enable invaluable parametric studies of the variables affecting the single cooling plume efficiency, in application, cooling holes are arranged in large grids of holes where the cooling film has an aggregate effect, which may not be a simple extension of the single cooling hole configuration. Installed applications of the film cooling holes typically use small diameter (on the order of $1 \mathrm{~mm}$ ) holes at inclined angles $(\alpha)$ to the plate surface, with L/D on the order of 3-4. Ligrani et al., (2013) performed a parametric study of contraction ratio, inclination angle, and blowing ratio on the film effectiveness for a dense array of staggered holes using thermocouple and infrared (IR) camera measurements. These studies were performed on a labscale rig with low velocity flows and freestream temperatures $<$ $115^{\circ} \mathrm{C}$, and concluded that film effectiveness leveled off at $\mathrm{BR}=2$. In another study, Ligrani et al., (2012) examined the effect of sparse versus dense cooling hole arrays and determined that the dense arrays provided higher film cooling effectiveness and higher heat transfer coefficients due to the complex interactions between the film and the boundary layer. They also determined that the dense hole array film effectiveness was less sensitive to BR than the sparse arrays.

Lihong et al., (2017) conducted numerical studies of dense cooling hole arrays over corrugated surfaces in order to determine the adiabatic film cooling effectiveness as well as heat transfer coefficient. Commercial software was used to grid and compute the momentum and heat transfer fields above the corrugated surface across an 8 million point grid. Mendez et al., 2006 performed a numerical investigation of the cooling film flow over a multi-perforated plate using a two stage approach. The flow exiting a single hole was initially modeled with high grid resolution. Periodicity was invoked and the single hole flow was replicated across the modeled domain and then the entire flow field solution was computed. Comparisons of a single hole solution with a 4 hole solution provided reasonable agreement, adding credibility to the approach, which is economical in overall grid complexity. Andrei et al., (2014) performed both numerical and experimental studies of the flow over a multi-perforated plate using Pressure Sensitive Paint to measure the adiabatic film effectiveness using an oxygen free injectant gas. Numerical simulations used an algebraic anisotropic correction to a $k$ $\omega$ Shear Stress Transport (SST) turbulence model in order to overcome the well-documented jet penetration over prediction and lateral spreading underestimation of classical RANS turbulence modeling. Furthermore, their experimental results showed that effective wall protection is achieved at low blowing ratios in the first part of the plate $\left(<6^{\text {th }}\right.$ row of holes) due to jet lift-off, however, further down the plate the aggregate injected cooling air protected the plate surface from the hot ambient flow.

In many of these past works on thin film cooling, the test hardware is limited to lab scale flows (velocity $<20 \mathrm{~m} / \mathrm{s}$ ) and small temperature differences or high density simulant gases are used so that the instrumentation can survive the environment [Mee 1999, Bergels et al., 1977, Andreopoulos, 1984]. Nearly all of these investigations used measurements of the material surface properties to estimate the film cooling effectiveness or the thermal heat transfer coefficient, which are prone to several error sources if not properly calibrated. Measuring the surface temperature is simply determining the "effect" of the film cooling and not the actual mechanisms by which the surface is being cooled. These lab scale flows also fail to capture all of the relevant physics that are present at the more realistic high temperature and much higher speed conditions of actual jet engines. One of the few realistic engine condition tests was performed on cooling turbine blades in a blowdown test rig utilizing heat flux gauges to measure heat transfer and surface temperatures but not the gas temperatures [Haldeman, 2006]. Another turbine blade cooling experimental test at transonic speeds using simulant high density gas was performed using liquid crystals to measure surface heat transfer [Poinsatte, 2008]. Besides pitot probe measurements [Bergeles et al., 1977], the only other off body measurements in thin film cooling flows have been made using hotwire anemometry, performed in low speed flows so that the probes will survive [Andreopoulos, 1984, Shyam et al., 2014].

Computational fluid dynamics (CFD) predictions at transonic speeds and high temperatures are handicapped by a lack of experimental data available to validate results, except for the work of Wernet et al., (2018). The typical industrial practice utilizing a RANS approach, employs a linear two-equation turbulence model, and a constant turbulent Prandtl number, $\mathrm{Pr}$, to calculate the turbulent heat flux terms in the energy equation [Yoder, 2016]. Surface heat transfer coefficients are 
important, and there are some empirical models that can be used for problems that are not too different from a known flow field. However, accurate prediction of relevant aerothermodynamics in a film cooling configuration involves complex momentum and heat transfer occurring in the shear layer between the large temperature and velocity gradients. Hence, the objective of this work is to acquire a comprehensive, high quality set of Raman temperature data and PIV measurements in a more realistic installed film cooling application. Traditional measurement techniques (pressure transducers and hotwire anemometry), and specifically those for temperature (thermocouples and hotwires), typically cannot survive in the hostile high-temperature, high-velocity flows of relevance. They are either severely restricted in temporal response, suffer from limited spatial resolution, or have a limited lifespan once in contact with the flows. Their accuracy is also questionable since they are intrusive to the flow and as such disturb and alter the very phenomenon that they are attempting to measure. Non-intrusive measurement techniques are the preferred approach for measuring the flow field properties under consideration here.

PIV is the defacto standard for measuring flow velocities and is readily applied in the high temperature, transonic flow as required in this work [Bridges \& Wernet, 2003, 2011]. While PIV systems are ubiquitous and commercially available, there are no such options for acquiring non-intrusive temperature measurements. Rayleigh spectroscopy has been used in jet flow facilities to measure the flow parameters such as velocity, density and temperature [Panda et al. 2005, Mielke \& Elam, 2009] however, while the velocity and density data were of good quality, the rms temperature data are of lower quality. This is believed to be the case because the measurements of temperature were frequently contaminated by entrainment of particulates in the flow. The Rayleigh technique also requires a complex optical system setup and is very sensitive to ambient light, flare light and vibration. Coherent Anti-Stokes Raman Spectroscopy (CARS) is a complex optical technique requiring precise alignment of three coincident laser beams and has been used to obtain average temperature in high speed and reacting flows. The CARS technique is also plagued by a very complex optical setup and the associated alignment issues thereby requiring the laser to be remotely located in a climate controlled room [Cutler et al., 2014, Tedder et al., 2009]. Transient Grating Spectroscopy (TGS) has also seen recent application to temperature measurement. While this technique has seen some success in temperature measurement within jet flows [Kuehner, 2010], it too suffers from a complex optical setup requiring precise alignment of multiple laser beams and as such is not amenable to large scale, outdoor facilities. Vibrational and rotational Raman spectroscopy are inelastic scattering techniques that have been used quite successfully to perform temperature measurements on a variety of flows. Single-shot vibrational Raman thermometry has been performed in flames [Ajrouche, 2014] where a fast optical shutter was used to minimize flame emission which would otherwise obscure or interfere with the SRS spectrum. Vibrational Raman is ineffective in low temperature regimes due to the lack of the anti-Stokes bands; however, pure rotational Raman spectroscopy, which is not dependent upon anti-Stokes lines, has been used to interrogate expanding supersonic flows of $\mathrm{CO}_{2}$ for measuring temperature [Maté et al., 1998]. Rotationally resolved Raman has more recently been used to acquire mean and rms temperature measurements in high temperature flows, from subsonic to supersonic speeds in harsh real world test facilities [Locke \&Wernet, 2017]. Rotationally resolved Raman scattering is the only technique which is relatively simple to setup/align and robust against the hostile environments found in real world aerospace simulation facilities; therefore it was the technique of choice for measuring the gas temperatures in thin film cooling - shear layer flows of interest in this work.

\subsection{Project Description}

NASA's Turbulent Heat Flux (THX) task under the Transformational Tools \& Technologies (TTT) project has been focused on acquiring benchmark velocity and temperature data in turbulent shear layers for validation of computational fluid dynamics (CFD) codes. The experiments described in this report are one of the only recent TTT-sponsored efforts where the turbulent transport of heat is a dominant aero-thermodynamic feature. Standard CFD turbulence models lack the ability to accurately calculate a number of fundamental flow phenomena, including the turbulent transport of heat. In nearly all production class Reynolds-averaged Navier-Stokes (RANS) CFD codes, a gradient-diffusion approximation is used whereby a constant turbulent Prandtl number, $\mathrm{Pr}_{\mathrm{t}}$, is used to relate an eddy viscosity calculated for the momentum terms, to the turbulent thermal diffusivity for the turbulent heat flux terms. The default in many CFD codes is $\operatorname{Pr}_{t}=0.9$, however this is not representative of all flows. As discussed in Yoder et al., (2015) and Reynolds, (1974), $\operatorname{Pr}_{t}=0.7$ is frequently considered a more appropriate single value choice for jets, but is also believed not to be a constant across the entire flowfield. Recent work, as discussed in Yoder, (2016), has explored more complex formulations including variable turbulent Prandtl number models, but results have not generated significant improvement over the constant $\operatorname{Pr}_{t}$ approach. A key difficulty in developing more accurate models for the turbulent heat flux is the lack of experimental data that quantify the turbulent thermal state. There have been minimal measurements made for turbulent heat flux problems outside of simple wall bounded flows [Kays, 1994]. The focus of the THX task has been to acquire datasets of $1^{\text {st }}$ and $2^{\text {nd }}$ order velocity statistics as well as mean temperature and root-mean-square (rms) temperatures with high accuracy and high spatial resolution. The THX Step I experiments were conducted in a small scale wind tunnel where Dual-Plane PIV data and hotwire data were acquired using both three and one heated injection air plume(s) into a low speed flow [Wernet et al., 2016, Borghi et al., 2018]. The work of Borghi et al., (2018) included LES modeling of the single cooling hole configuration. The THX Step II tests consisted of acquiring Raman temperature measurements, using the same 
laser/optical hardware applied in the work reported here, in the jet flow field issuing from a $50.8 \mathrm{~mm}$ diameter heated convergent nozzle operated over a range of temperatures and Mach numbers [Locke \& Wernet, 2017]. The Raman temperature data were collected to be used in conjunction with an existing database of PIV data [Bridges \& Wernet, 2011] that is used by the RANS turbulence modeling community [Rumsey et al., 2010]. Recently, this new temperature data set enabled assessment of LES predictions of flow velocity, turbulence, mean temperature and rms temperature [Debonis, 2017]. In Step III of the THX task, both velocity field and temperature data were acquired for a cooling configuration with a relatively large single cooling hole [Wernet et al., 2018]. The geometry was simple enough to permit detailed velocity and temperature measurements (mean flow and turbulence) to characterize the fluid dynamics and thermodynamics in the vicinity of the interaction region between the hot main flow stream and the injected cooling flow.

The work presented here is Step IV of the THX task, where a new cooling configuration is employed that more closely resembles an actual turbine blade cooling treatment or aircraft afterbody cooling arrangement. The same heated square nozzle jet flow as used in Step III was used to deliver hot flow over the plate, but now the plate was equipped with three patches of 45 cooling holes positioned within the first half of the length of the plate. The motivation was to obtain detailed aerothermodynamic data in the vicinity of the interaction regions of the film cooling introduced through the 3 separate patches of cooling holes with the primary flow stream which is heated. The following flow measurements were acquired: (1) Background Oriented Schlieren (BOS) for the cooling film thickness on the plate, (2) PIV for velocity statistics, (3) rotational Raman scattering for off-body temperature data, (4) thermocouple surface temperature measurements and finally, (5) IR camera measurements of the full plate surface. The objective of the Step IV test described herein was to collect both mean and rms velocity and temperature statistics via PIV and rotationally resolved Raman spectroscopy across a precisely defined measurement grid in the plume of a convergent hot jet. The acquired data should be useful for assessment and improvement of CFD turbulent calculation methods since regions of high turbulence are assumed to be the regions of peak fluctuations in temperature. Additionally, these film cooling flows will contain interacting streams of gas at different temperatures moving at different velocities thereby exhibiting both momentum and thermal transfer processes, which eventually must be accurately modeled via CFD.

The AeroAcoustic Propulsion Laboratory (AAPL) at NASA GRC was selected for this series of tests since it can provide the heated and injection air flow streams necessary for simulating thin film cooling. While the test article employed an "external" flow of sorts that is more amenable to optical measurements than an "internal" flow, the AAPL has a number of features that make implementing optical diagnostics a challenge: 1) The AAPL is open to the outside environment and as such experiences large temperature variations over the course of a day-long test, 2) High sonic noise levels upwards of $115 \mathrm{~dB}, 3$ ) High ambient light levels due to the large, 55' wide by $36^{\prime}$ high exhaust-door which must remain open during tests, and 4) Inaccessibility by personnel during the tests. Taking all of the above into consideration, Rotational Raman was selected as the temperature diagnostic for this test due to the relative simplicity of its installation and robustness against hostile environments (temperature variations and high vibration levels) compared to the complex and sensitive alignment requirements for the other optical techniques described above. Likewise, PIV was selected as the flow measurement diagnostic for velocities due to our longstanding experience in applying PIV in the Small Hot Jet Acoustic Rig (SHJAR) facility at GRC [Bridges and Wernet, 2003, 2011].

\subsection{AAPL Laboratory, SHJAR and Experimental Setup}

All the data presented herein were obtained on the SHJAR located within the AAPL at NASA GRC. The AAPL (Fig. 1) is a $19.8 \mathrm{~m}$ radius geodesic dome with its interior walls covered by sound absorbing wedges providing a near anechoic environment. The SHJAR (Fig. 2) is a single flow stream free jet rig capable of operating over a range of Mach numbers up to $\mathrm{M}=2$ at jet static temperature ratios up to approximately 2.8 for a nozzle with Mach 1 exit condition. The centerline of the nozzle exit of the SHJAR is $3 \mathrm{~m}$ above the floor. Vitiated flow heating up to $950 \mathrm{~K}$ is provided by an inline hydrogen combustor and supply air is provided by central compressor facilities, permitting continuous operation. The fuel-air mass flow ratios for heating the supply air ranged from $2.8 \times 10^{-4}$ to $7.2 \times 10^{-3}$ resulting in a small change in the nitrogen to oxygen ratio of the heated air at the nozzle exit and over the flat plate, but not large enough to cause any significant difficulties in estimating the gas temperature.

The SHJAR facility is frequently used for PIV measurement studies where aluminum oxide is used to seed the flow. Raman scattering is a molecular scattering technique and as such any particulates in the flow are detrimental to the quality of the acquired Raman signals, hence, simultaneous velocity and temperature measurements were not feasible. As an additional preparation for the test, the SHJAR was disassembled and cleaned of any residual seed material coating the inside flow passages of the rig/model hardware from previous tests. The Raman portion of the test program was conducted first, followed by the PIV measurement campaign, with the requisite particulate seeded flow streams. 


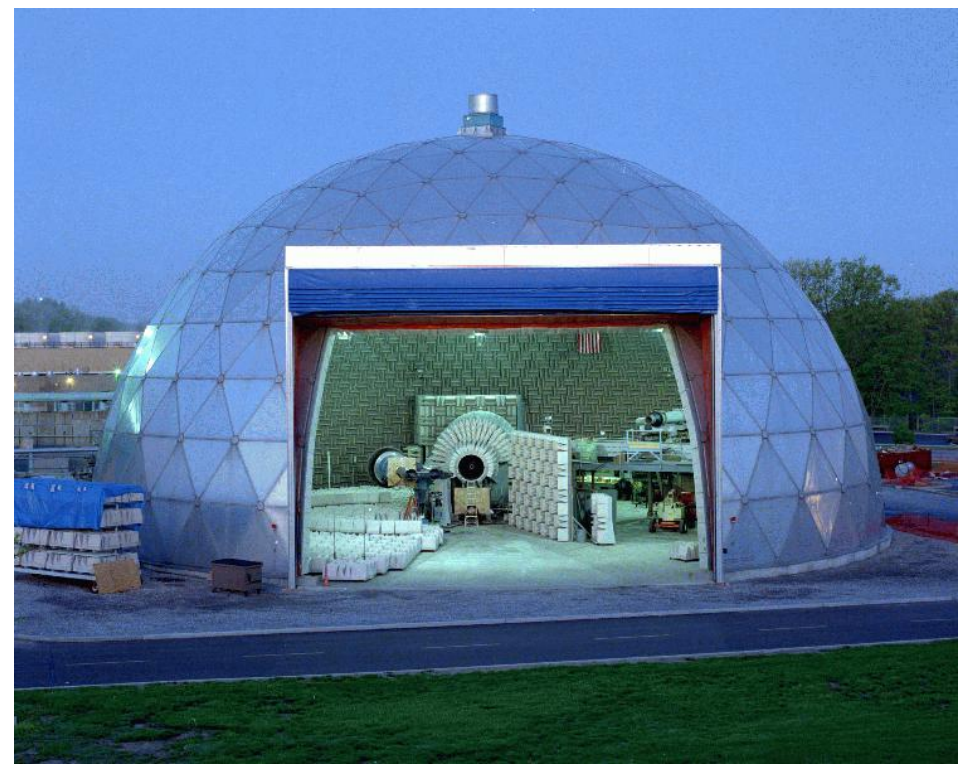

Figure 1. Photograph of the AAPL laboratory. SHJAR is the smaller rig to the left of center.

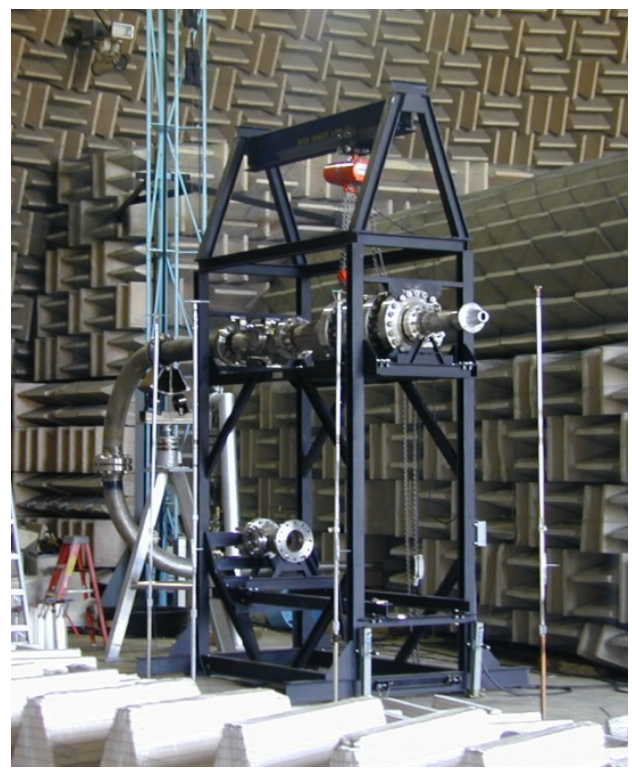

Figure 2. Photograph of the SHJAR Rig.

The THX Step IV test utilized the same square nozzle used in the THX Step III test. The nozzle transitions from a 152.4 $\mathrm{mm}$ radius round cross-section through a series of super ellipses to a square nozzle with $68.07 \mathrm{~mm}$ sides at the nozzle exit. This square nozzle exit shape was chosen in order to provide a volume of uniform flow over the plate surface where the hot exhaust interacted with the injection stream. The dimension of $68.07 \mathrm{~mm} \times 68.07 \mathrm{~mm}$ exit area was determined to be the largest that would correspond to a nozzle mass flow that would not overly strain the flow and fuel supply systems of the SHJAR. See Figs. 3 and 4 for schematic drawings of the test hardware. The nozzle internal contour was designed to have a continually decreasing cross sectional area while moving downstream, to enable a favorable pressure gradient, and in turn, minimal secondary flows at the nozzle exit. A flat plate or deck was mounted to the square nozzle to form the nozzle/deck assembly, see Fig. 3. The $6.35 \mathrm{~mm}$ thick plate spans the width of the nozzle exit $(68.07 \mathrm{~mm})$ and extends $304.8 \mathrm{~mm}$ axially from the nozzle exit, ending with a $15^{\circ}$ taper. The plate was equipped with three separate patches of 45 holes arranged in a staggered pattern, as shown in Fig. 4 with intra-hole spacings of $\Delta X / D=5$ and $\Delta Z / D=5$. The Zoffset of each successive row is $\mathrm{Z} / \mathrm{D}=1.25$. The cooling holes were $1 \mathrm{~mm}$ diameter and inclined $30^{\circ}$ from the horizontal, yielding an $\mathrm{L} / \mathrm{D}=12.5$. The cooling air flow was delivered to the plate via a dual chambered plenum assembly, in order to provide a uniform cooling air supply from the bottom side of the perforated plate at a constant blowing ratio for the three patches of holes. The unheated shop air cooling flow entered the high pressure bottom plenum through a $12.7 \mathrm{~mm}$ orifice. A choke plate with thirty-two $0.78 \mathrm{~mm}$ diameter holes arranged in a $4 \times 8$ regular grid pattern $(\mathrm{X} / \mathrm{D}=\mathrm{Z} / \mathrm{D}=25.8)$ was used to provide a uniform flow and pressure field below the cooling holes in the top plate. The height of the low pressure top plenum was designed to provide adequate distance for the individual jets from the choke plate to mix out into a uniform flow. The highest pressure differential observed across the choke plate was 10:1 for the highest blowing ratios used in this study. The top and bottom plenums were designed with $6.35 \mathrm{~mm}$ thick walls to ensure structural integrity of the model under the full range of blowing ratios. In order to accommodate the $6.35 \mathrm{~mm}$ plenum walls, the top chamber was wider than the top plate surface. This resulted in the $45^{\circ}$ bevel along the sides of the plate. The cooling flow originated from a plenum box, where unheated shop air (at nominally the ambient air temperature) mixed with PIV seed particles before entering the lower plenum (no seed was used for the Raman temperature measurements).

A total of 17 thermocouples were embedded along the length of the plate extending from the nozzle exit, shown as the red dots in Fig. 4. Holes were drilled from the underside of the plate leaving just $0.762 \mathrm{~mm}$ of material between the thermocouple and the top surface of the plate. Five rows of 3 thermocouples were installed at the following locations: $X$ $=21.3,83.5,145.8,208.0$, and $270.3 \mathrm{~mm}$, where $X=0 \mathrm{~mm}$ is at the nozzle exit. At each of the 5 axial locations, three thermocouples were located at span locations of 0.0 , and $\pm 19.05 \mathrm{~mm}$, with $Z=0$ being the spanwise plane of symmetry, passing through the center of the plate. Two thermocouples were also embedded at $X=56.9 \mathrm{~mm}$ and $119.1 \mathrm{~mm}$ at $Z=0$ $\mathrm{mm}$. The last thermocouple was embedded in the nozzle, at $X=-6.4 \mathrm{~mm}$ and $\mathrm{Z}=0 \mathrm{~mm}$. In addition to the embedded thermocouples, 2 surface mounted thermocouples were spot welded to the bottom surface of the plate, at axial stations of $X=56.9 \mathrm{~mm}, \mathrm{Z}=12.7 \mathrm{~mm}$ and $\mathrm{X}=119.1 \mathrm{~mm}, \mathrm{Z}=-12.7 \mathrm{~mm}$, as denoted by the open blue circles in Fig. 4. Thermocouples and pressure taps were also inserted into the bottom and top plenums to measure the cooling air flow 
temperature and pressure. The seed mixing plenum was also equipped with a thermocouple in order to monitor the air temperature inside the chamber. The SHJAR facility data acquisition system was used to record all of the rig operating temperatures and pressures during the testing. At the beginning of each data acquisition sequence (BOS, Raman or PIV) the rig settings were recorded. The average thermocouple readings of the embedded and surface mounted thermocouples were processed and tabulated.

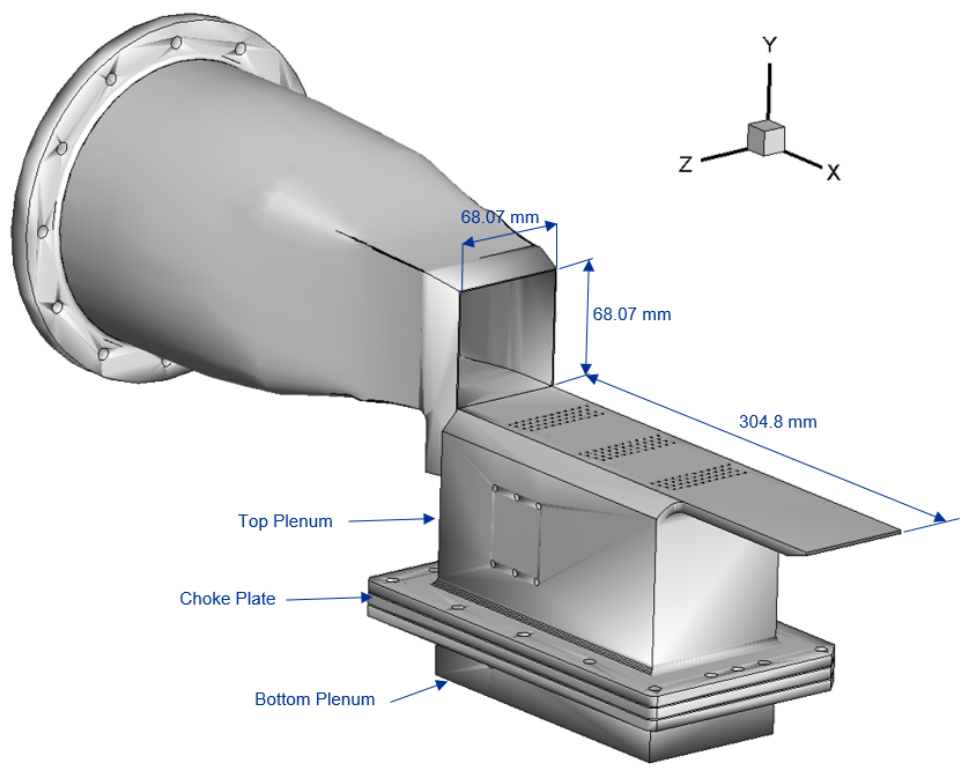

Figure 3. Experimental configuration - square nozzle with the $6.35 \mathrm{~mm}$ thick plate and three patches of forty-five $1 \mathrm{~mm}$ diameter injector holes angled at $30^{\circ}$ to the plate surface. Cooling air is provided by the two chamber plenum.

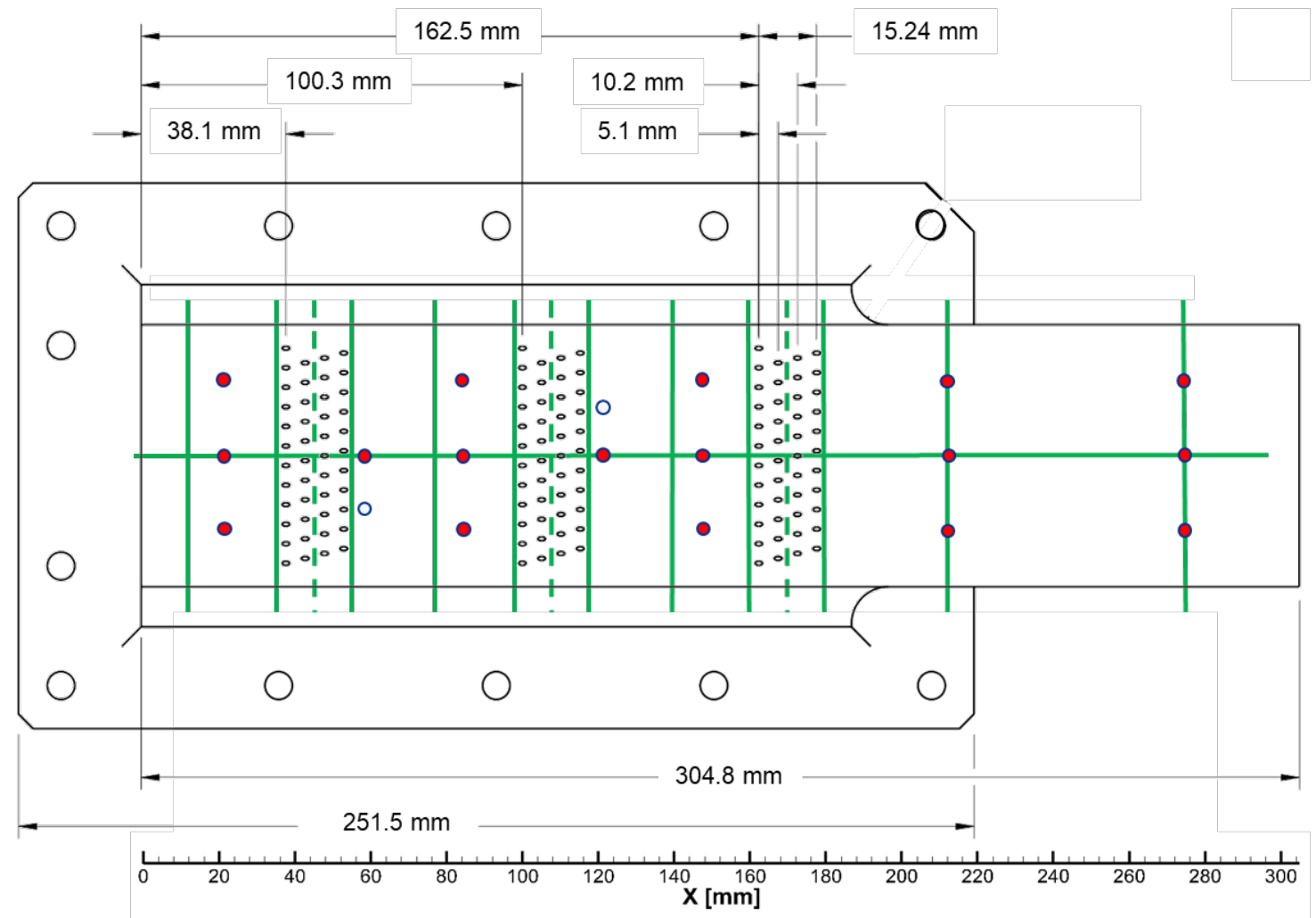

Figure 4. Top View of the plate showing the cooling hole geometry and dimensions. The red circles indicate the thermocouple locations embedded in the top surface of the plate. The empty blue circles indicate the locations of the thermocouples on the underside of the plate. The green lines show the planes where PIV data were collected. Raman temperature surveys were performed at the intersections of the solid green lines and the centerline streamwise PIV plane. The scale on the bottom provides a reference for the axial locations of the Raman, thermocouple, and PIV measurements. 


\subsection{Pure Rotational Raman Temperature Measurements}

Rotationally resolved Raman scattering has been previously used to measure gas temperature in heated high speed jet flows in the SHJAR facility at NASA GRC [Locke et al., 2017]. A thorough discussion of Raman spectroscopy theory and practices can be found in Ferraro and Nakamoto [18]. In general, Raman scattering is an inelastic process, with a signal intensity approximately $10^{-3}$ of that from Rayleigh scattered light. Raman scattering is not dependent on wavelength but is linear with respect to the species number density and is species specific by virtue of the quantization of individual molecular energy states. Here we are not concerned with determining the composition of the gas being probed (heated air), just the gas temperature, which greatly simplifies the data analysis and reduction.

The key features in the layout of the Raman temperature diagnostic are shown in Fig. 5. A 10Hz Continuum long pulse length Agilite Nd:YAG laser with $600 \mathrm{~mJ}$ of energy at $532 \mathrm{~nm}$ spread across $200 \mathrm{nsec}$ was used to probe the flow temperature and still avoid breakdown of the gas. The $9 \mathrm{~mm}$ diameter output beam was focused by a $500 \mathrm{~mm}$ spherical lens to a roughly $70 \mu \mathrm{m}$ beam waist. The rotational Raman scattered signal was collected by two matched f/1.4, $135 \mathrm{~mm}$ Nikon lenses set at $\mathrm{f} / 4$ and equipped with $42 \mathrm{~mm}$ extension tubes. A pair of collection lenses was used in order to maximize the collected signal. The lenses were vertically mounted and focused on the laser focal volume approximately $257 \mathrm{~mm}$ distant. Each lens was displaced from the horizontal plane and tilted by $8.5^{\circ}$ (upper $-8.5^{\circ}$, lower $+8.5^{\circ}$ ), which is the closest the lenses could be mounted side-by-side.

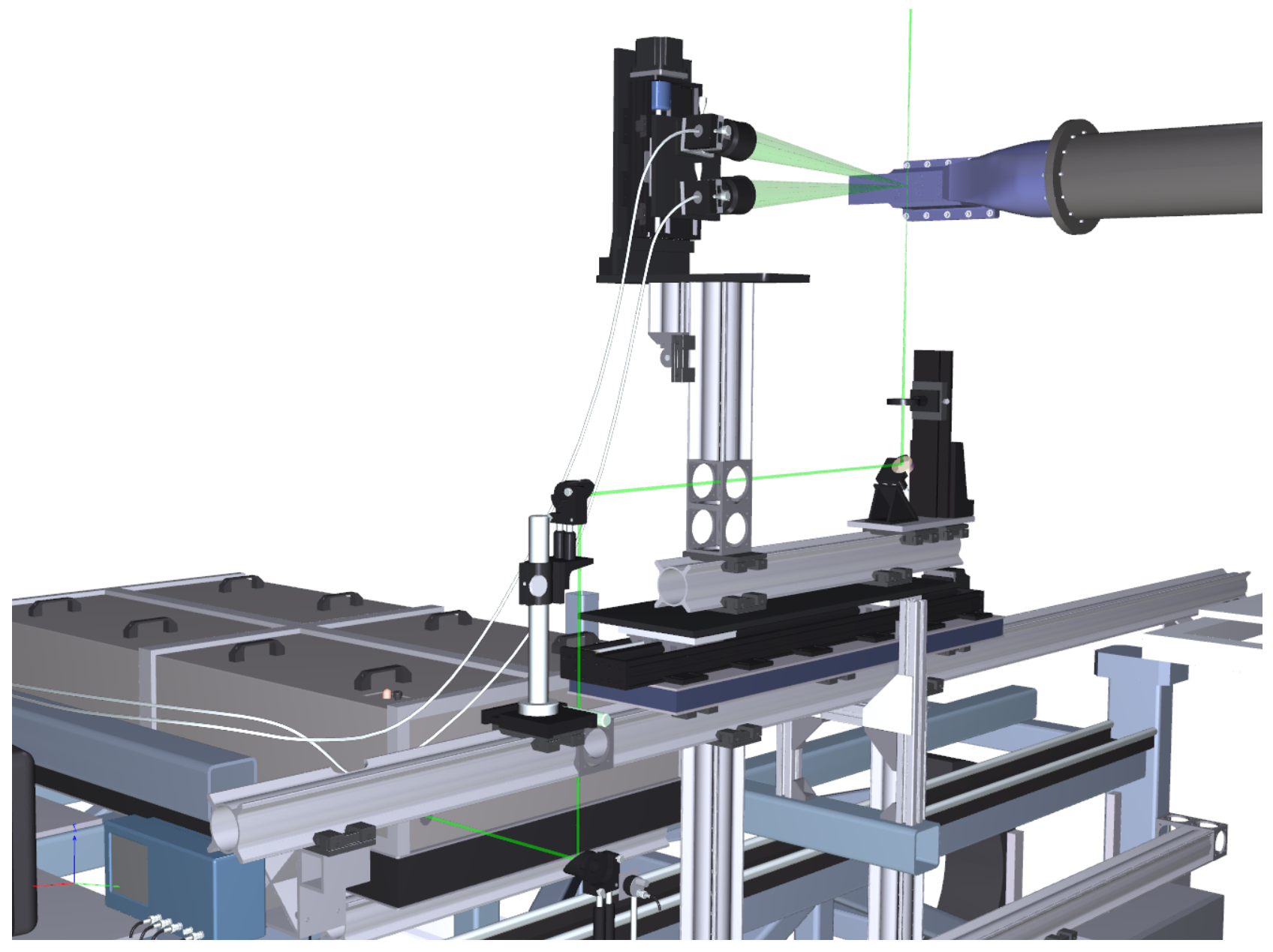

Figure 5. Schematic of the rotational Raman collection optics as installed in the SHJAR facility.

The light captured by each lens was passed through a $532 \mathrm{~nm}$ RazorEdge long-pass $24.5 \mathrm{~mm}$ filter attached to the rear of each camera lens mount. The filtered light was focused onto a bifurcated fiber bundle from Fiberoptic Systems, Inc. The input ends of the bifurcated fiber bundle each contained fifty-seven $100 \mu \mathrm{m}$ diameter cladding-free fibers which were formed into a linear array at the output end of the bundle. The input end of the collection fiber bundles had the fibers arranged in an ordered array of concentric rings. The outer most ring of fibers at the collection end was oriented at the top of the linear array end of the fiber (entering the spectrometer). The $2^{\text {nd }}$ outer most ring of fibers on the collection end 
of the bundle were then positioned just below the first set of fibers in the linear output. The linear fiber bundle output was coupled to the entrance slit of an Acton $500 \mathrm{~mm}$ imaging spectrometer with an $1800 \mathrm{gr}$ ruled grating and a wavelength centerline of $537 \mathrm{~nm}$ resulting in a spectral wavelength span of approximately $12 \mathrm{~nm}$. Since the $100 \mu \mathrm{m}$ fibers of the transmission bundle were arranged linearly at the slit, the slit of the spectrometer was opened to its maximum of $2.0 \mathrm{~mm}$ allowing the fibers to act as their own $100 \mu \mathrm{m}$ slit. A PI-MAX2 ICCD camera with $18 \mathrm{~mm}$ diameter gated intensifier from Princeton Instruments was coupled to the exit of the spectrometer and the resultant rotational Raman spectra captured using Princeton's WinSpec32 software. In order to verify alignment of the $532 \mathrm{~nm}$ laser beam with the focus of the two camera lenses, a $635 \mathrm{~nm}$ diode laser was fiber-coupled to the rear of the two $85 \mathrm{~mm}$ collection lenses and directed back to the probe volume, where the alignment of all three components of the measurement system could be visually confirmed by the intersection of the back projected/focused diode laser beams. The Raman measurement volume is defined by the intersection of the laser beam diameter and the two $135 \mathrm{~mm}$ lens collection cones. The length of the $70 \mu \mathrm{m}$ diameter beam collected by the $135 \mathrm{~mm}$ lenses is defined by the size of the fiber bundle used to collect and transmit the light to the spectrometer. The entrance face of each of the fiber bundles is $0.85 \mathrm{~mm}$ in diameter. The $135 \mathrm{~mm}$ lenses image a 1.2 $\mathrm{mm}$ length of the laser beam onto the face of the fiber bundle. The resulting cylindrical probe volume used in this work is $4.5 \times 10^{-3} \mathrm{~mm}^{3}$. Due to the ordered array of fibers on the inlet and output ends of the fiber optic bundle, the effective size of the probe volume could be adjusted in software by restricting the region of interest of the CCD image from the spectrometer. If the outer ring is cropped in the data processing, the probe volume length is reduced to $1.0 \mathrm{~mm}$. If the two outer rings are cropped, the probe volume length is reduced to $0.7 \mathrm{~mm}$. For this work, the full image area on the CCD sensor was used, i.e. a $1.2 \mathrm{~mm}$ long probe volume.

\subsection{Raman Data Processing}

To extract temperature from the rotational Raman spectra, an iterative process was developed which determines the best fit between the measured spectrum and the spectrum computed using a pure-rotational Raman scattering model, which is described in more detail in Locke et al., 2017. To model the Raman scattering spectrum, an assumption is first made that the gas composition is a mixture of only molecular nitrogen and oxygen. This can be done since the measurements are made in air and other atmospheric gaseous components do not contribute significantly to the Raman signal. For a given temperature, the model computes Raman line locations and strengths in the Raman Stokes (S) bands of nitrogen and oxygen up to rotational quantum numbers of 50. The merged array of Raman lines for $\mathrm{N}_{2}$ and $\mathrm{O}_{2}$ with their respective strengths and wavenumbers, is then convolved with a Voigt kernel derived from the spectral profile of the pump laser wavelength peak as measured through the optical system.

In the data processing stage, the 1000 single-shot spectra were read into a Matlab-based data reduction software. In each acquisition sequence, there were typically several bad spectra due to the wait time of the camera/spectrometer/image intensifier. Additionally, there were times when particulates in the flow passed through the measurement volume producing strong signals at the laser line wavelength, which were strong enough to yield Rayleigh/laser line peaks of higher intensity than the Raman signal. In order to remove these spurious spectra, the data set was sorted to remove the 10 highest amplitude spectra, hence only 990 single shot spectra were used in each ensemble.

The 990 spectra were then used to compute an average spectrum, which was fit to the $\mathrm{O}_{2} / \mathrm{N}_{2}$ model function using a combined genetic algorithm for the global search followed by a local search using a non-linear least squares (NLLS) routine. The model parameters used in the fit included the temperature, the Raman signal amplitude, the kernel width (a 2-parameter Voigt instrument function) and the spectrometer grating calibration parameters, which included the laser line center location. The fit of the mean spectra provided the initial estimates for all of the single shot spectral fits. The estimated temperatures from the processed spectra were then used to compute the mean temperature estimate and the rms temperature across the ensemble, $\mathrm{T}^{\prime}$. The time to process the 990 spectra was on the order of 1 minute on a 20-core CPU. A sample averaged spectrum acquired at 547K and its corresponding fit are shown in Fig. 6.

\subsection{Raman System Calibration}

The Raman technique samples the molecules in the probe volume in order to estimate the gas temperature. The molecules in the probe volume can have any one of a number of states as defined by the Boltzmann distribution. The number of populated states increases with temperature, hence the rms temperature increases with increasing temperature of the gas. This is a known and documented characteristic of the technique, which is actually a systematic measurement error [Locke et al., 2017]. A calibration of the Raman system in a well characterized environment free from flow turbulence of other noise sources is required in order to characterize this inherent rms temperature variation in the technique. A lab scale setup using the concentrated output from an electrical heat gun calibrated using a thermocouple was used to acquire the calibration data [Locke et al., 2017]. The Raman spectra acquired at the 11 different temperature settings on the heat gun 


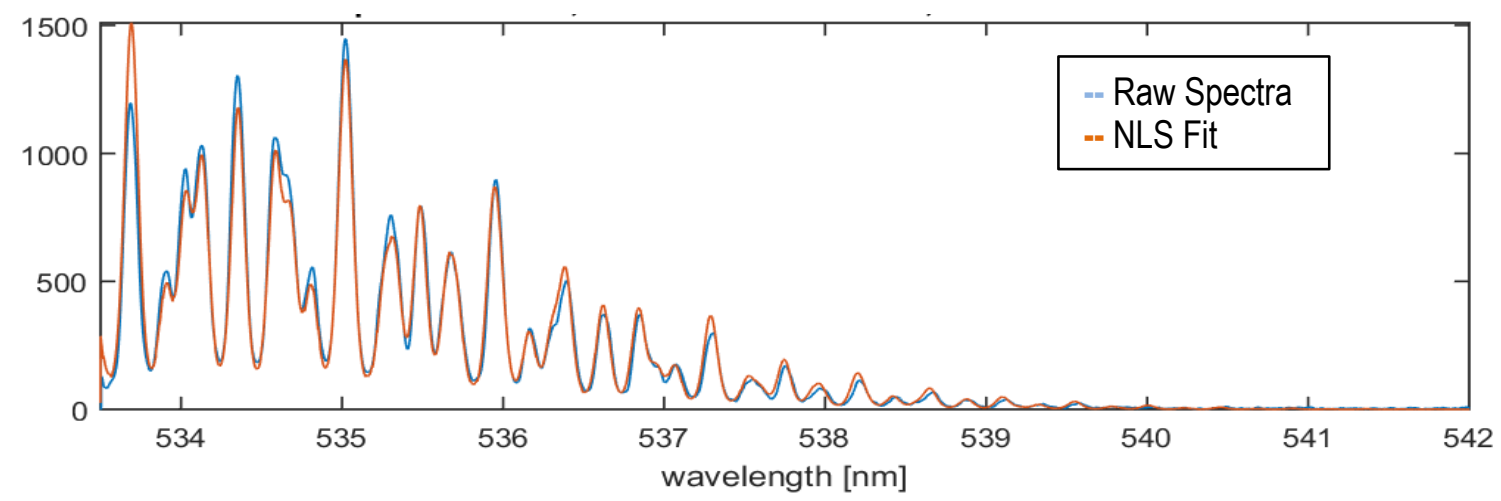

Figure 6. Sample averaged rotational Raman temperature spectrum with fit for a gas temperature of $547 \mathrm{~K}$. The spectrum is elongated in the $\mathrm{x}$-axis to show detail.

were processed according to the procedures described in the preceding data reduction discussion. The ensemble of 990 measurements at each point were used to compute the mean and rms gas temperature. Fig. 7 plots the mean temperature of all 990 accumulated spectra along with the rms error bars at each temperature. The mean calibration temperature measurements are found to be accurate to $<0.6 \%$ over the range of $296 \mathrm{~K}-850 \mathrm{~K}$. Here accuracy is defined as the deviation of the measurement from the true (known) value. For these calibration measurements, the mean temperature level is measured using a thermocouple. The reported error is the deviation of the Raman based mean temperature measurement from the thermocouple measurements. A full system calibration is required for each new configuration of the Raman diagnostic system. The system configuration in THX IV is using new collection lenses and a new bifurcated fiber optic imaging bundle compared to those used in the THX III testing program.

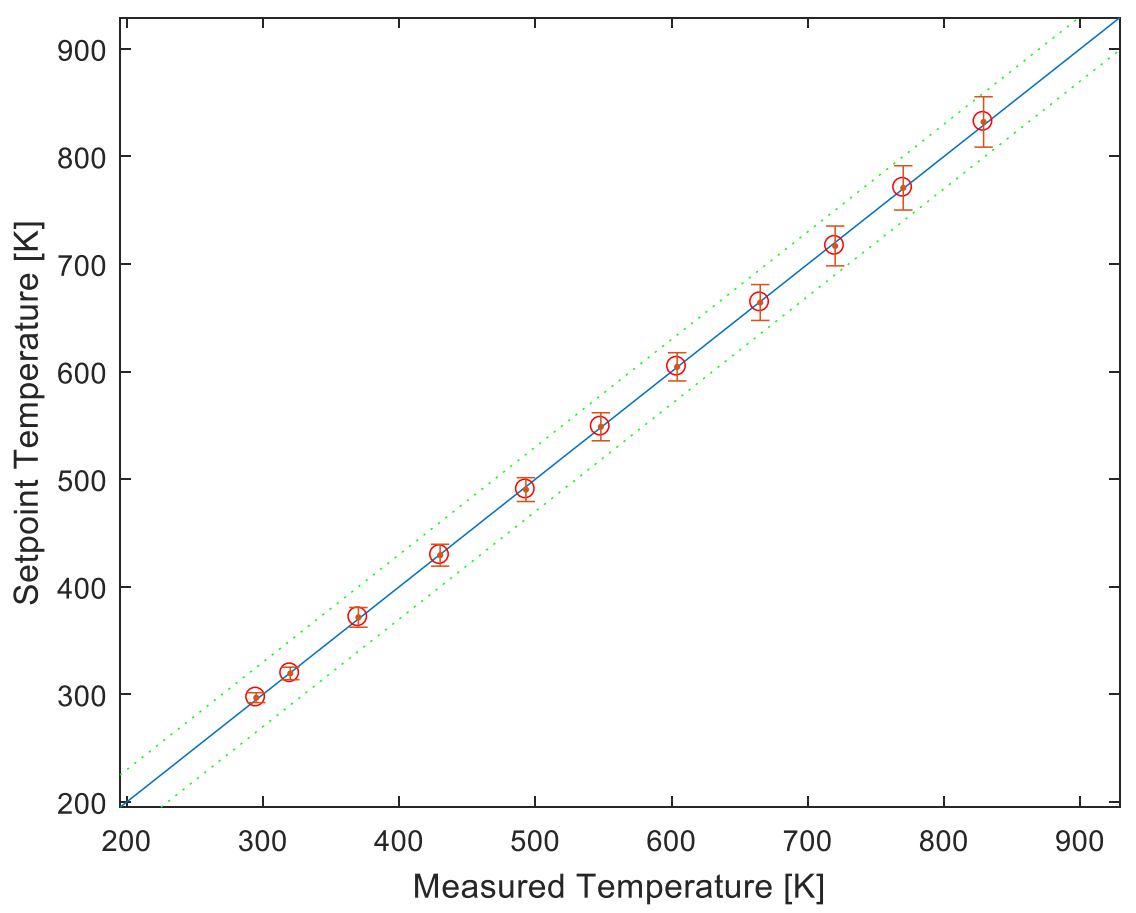

Figure 7. Plot comparing the thermocouple reading versus the calculated temperature and the RMS variations (plotted as error bars) in temperature over the range of calibration temperature range settings.

The Raman based calibration rms temperature estimate is $\pm 0.7 \%$ at $296 \mathrm{~K}$ and $\pm 0.4 \%$ at $850 \mathrm{~K}$. The Raman temperature measurements reported here illustrate the best case measurement accuracy that can be expected using the Raman Thermometry diagnostic. The Raman measurements contain 3 main contributions to the rms variations: 1) random measurement error $\left.\sigma_{M E}, 2\right)$ thermal fluctuations in the gas $\sigma_{T F}$, and 3) systematic error in the Raman diagnostic $\sigma_{R E}$, as shown in equation 1. These error sources add in quadrature to yield the total rms variation in the measured temperature. The large ensemble of 990 is used to drive down the random measurement error by $1 / \sqrt{ } \mathrm{N}$. Larger samples sizes were collected, but yielded no further reduction in the observed rms variations. Dynamic thermocouple measurements in the 
heat gun flow showed that the $\sigma_{T I}$ is negligible for the calibration measurements [Locke at al., 2017]. Hence, the remaining contribution to the observed rms variation is the inherent systematic error in the Raman technique.

$$
\sigma_{T}^{2}=\sigma_{M E}^{2}+\sigma_{T F}^{2}+\sigma_{R E}^{2}
$$

The calibration plot error bars in Fig. 7 clearly illustrate that the measured rms increases linearly with temperature, as stated above. A linear fit to the rms temperature as a function of the known temperature yields a relation for the expected systematic error in the Raman based temperature diagnostic:

$$
\sigma_{R E}=0.0303 T-4.88 \mathrm{~K}
$$

This expression will be used later to predict the expected systematic error resulting from the Raman temperature measurement technique when we examine the rms temperature measurements in the heated jet, cooling film flows.

\subsection{Raman System Installation in SHJAR}

The optical excitation and detection system used to acquire the Raman temperature laboratory calibration was also used in the performance of actual testing in the AAPL. The long-pulse, Agilite laser, beam insertion optics, detection optics and spectrometer/camera detection systems were transported, installed, and aligned in the AAPL as shown in Fig. 8. Note that the plate with the patches of cooling holes was mounted with its plane perpendicular to the floor in order to provide convenient optical access for the long pulse laser beam. Due to its size, the Agilite laser had to be mounted inside the frame of the large traverse system, which drove the film cooling plate orientation. The beam from the laser was directed towards the front face of the large traverse where it was turned to the vertical using a mirror. A catching mirror then turned the laser beam horizontal and parallel to the front face of the large traverse. A final turning mirror turned the laser beam vertical so that it passed vertically through the jet flow field. A $500 \mathrm{~mm}$ focal length spherical lens then focused the beam at the center of the flow, downstream of the nozzle's exit plane. The final turning mirror, lens and the camera collection lenses are mounted on a long Velmex translation stage which provides the transverse surveys of the jet/plate cooling flow.

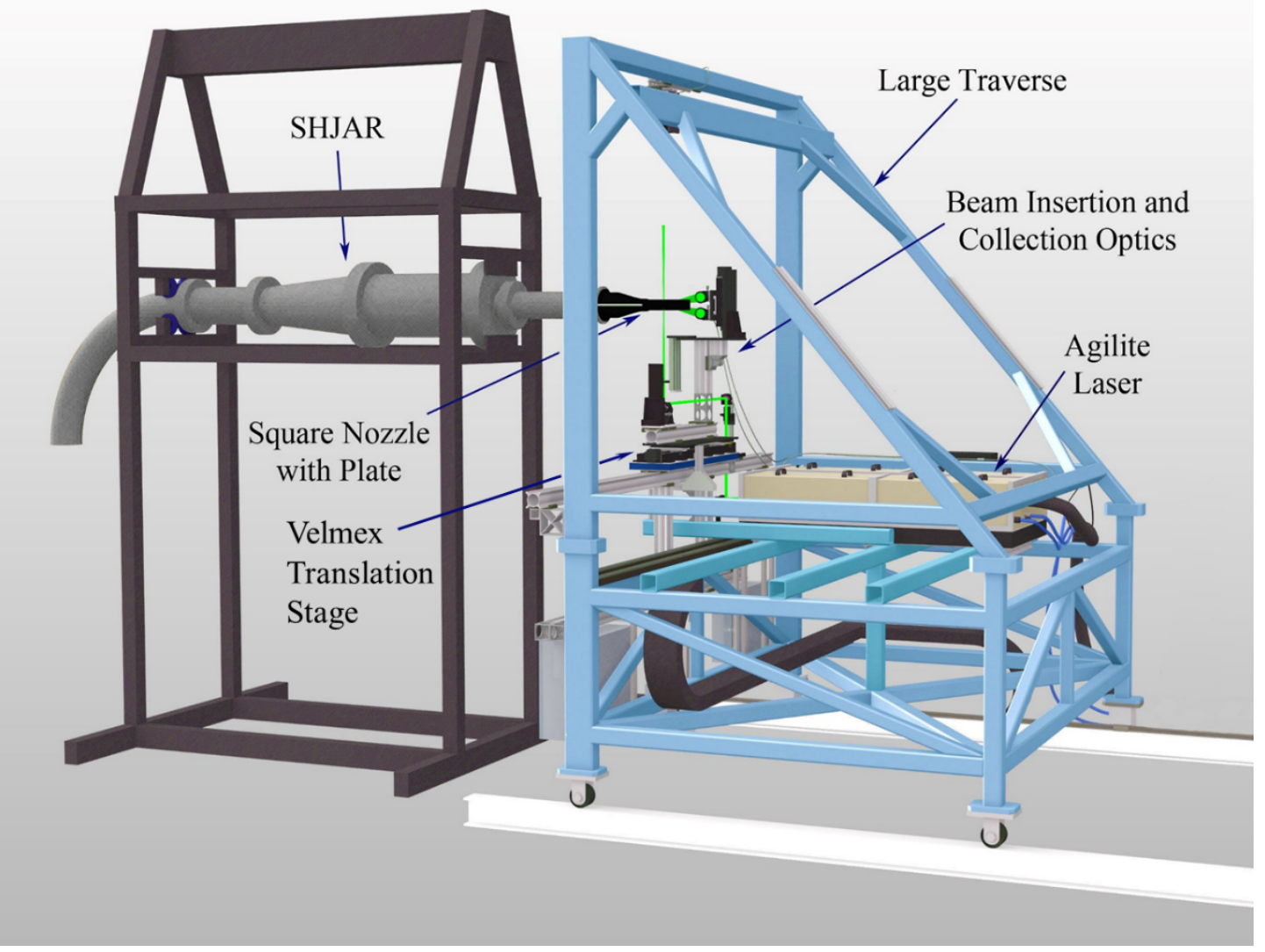

Figure 8. SHJAR and large blue traverse system loaded with the long-pulse laser and optical diagnostics. 
The vertical position of the measurement volume was adjusted using a second Velmex translation stage. The vertical translation stage adjusted the position of the collection lenses along the focused laser beam. The focus of the laser beam was not adjusted for the vertical translations, which spanned a $50.8 \mathrm{~mm}$ region along the beam waist.

\subsection{PIV Measurement System}

For the PIV portion of the measurement campaign, the plate with the patches of injection holes was mounted parallel to the ground. Data were acquired using two different PIV configurations: one mapping cross-stream planes, with the cameras mounted in a stereo configuration to obtain 3-components of velocity; and another mapping the nozzle/plate centerline plane using a dual side-by-side camera configuration to obtain 2-components of velocity.

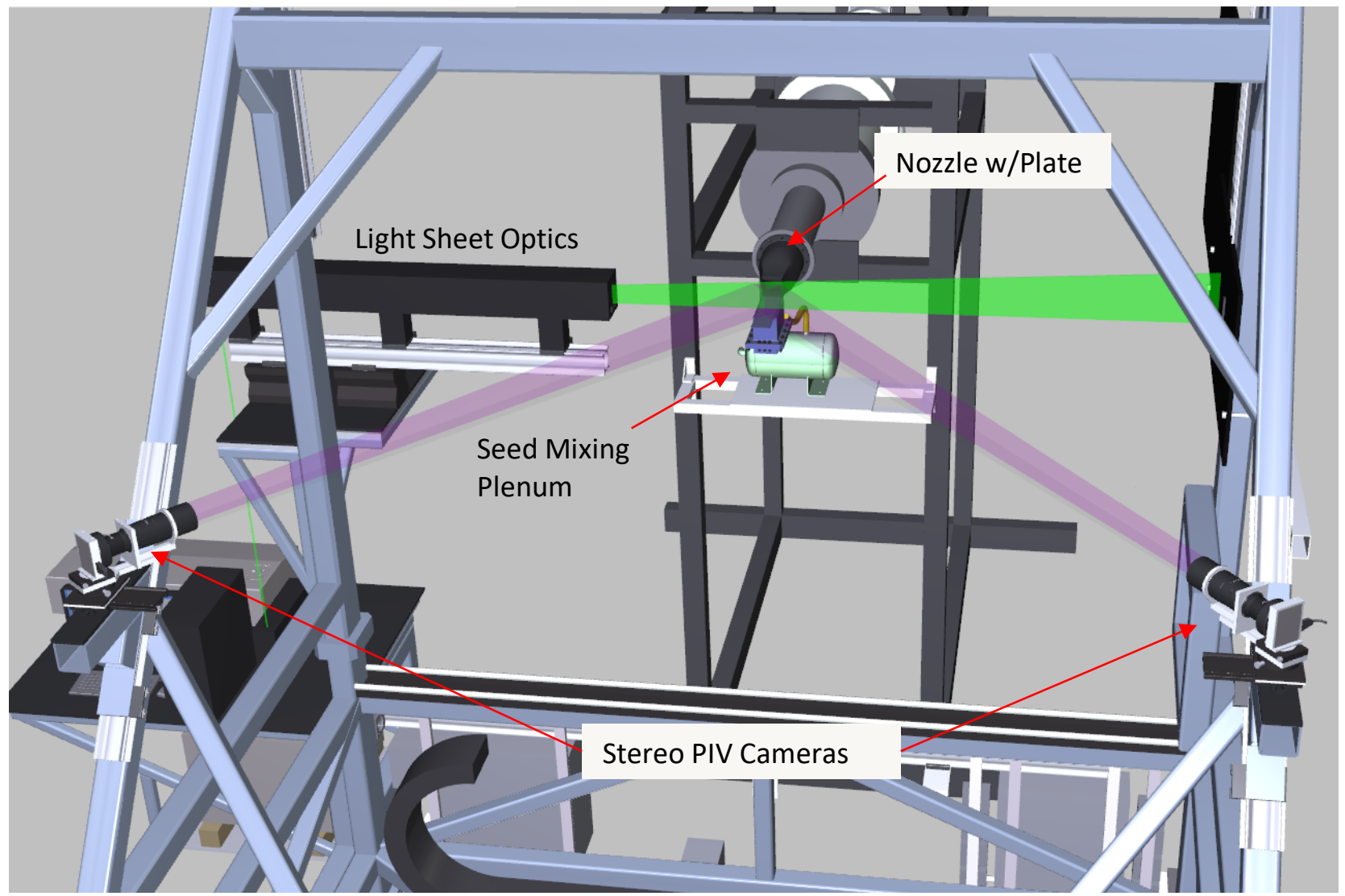

Figure 9. Cross-stream Stereo PIV installations on the large blue traverse system in the SHJAR.

\subsection{3-C SPIV Configuration}

The Stereo Particle Image Velocimetry (SPIV) system was configured to provide cross-stream measurements of the three-component (3C) velocity field from the test article. The entire SPIV system was mounted on the large traverse system to perform surveys of the flow field as shown in Fig. 9. The travel range of the traverse was approximately $2.133 \mathrm{~m}$, with a positioning accuracy of $1 \mathrm{~mm}$. The SPIV system employed two high-resolution (4008x2672 pixel) cameras equipped with $300 \mathrm{~mm}$ focal length lenses, to provide a 100x100 mm field-of-view. The cameras were mounted downstream of the model exit plane at nominally $\pm 45^{\circ}$ from the nozzle centerline. Both cameras were connected to a single computer system via a CameraLink PCI card and the 400 frame pair data sequences were acquired and streamed to disk at a rate of 2 frame pairs/camera/sec. Stereo PIV calibrations were performed using a single plane target translated to 9 axial positions over a $\pm 4 \mathrm{~mm}$ range. A $3 \mathrm{rd}$ order polynomial was used in the image warping and a calibration verification operation was employed to insure that the calibration overlapped the laser light sheet plane. The light sheet forming optics contained a clipping plate used to truncate the bottom edge of the light sheet as it crossed the plate surface. The laser sheet blocking plate was mounted on a translation stage so that the vertical height of the laser light sheet could be adjusted at the Stereo PIV 
axial measurement stations, since the test model was not perfectly level.

\subsection{2-C Streamwise PIV Configuration}

A standard PIV system was used to measure the 2-component (2C) streamwise velocity field down the centerline of the plate. In order to maximize the field of view while maintaining high spatial resolution PIV vector maps, a dual side-by-side camera configuration was used to acquire the centerline streamwise plane of data, as shown in Fig. 10. By orienting the $4008 \times 2672$ pixel PIV cameras in landscape mode (4008-pixel axis oriented horizontally) the entire length of the plate could be imaged without traversing the PIV system, shown as the centerline plane in Fig. 11. The cameras were equipped with $135 \mathrm{~mm}$ focal length lenses with $8 \mathrm{~mm}$ extension tubes and positioned so that their fields of view overlapped by $25.4 \mathrm{~mm}$. A flat, SPIV-style calibration target was used to calibrate and register the two cameras using a fiducial mark in the overlapping region of each cameras' field of view. The physical registration of the two cameras was used in the setup of the vector processing grids in the left and right camera images so that no interpolation was required in the merging of the left/right vector maps. The final merged camera vector map covered an area of $81 \times 304 \mathrm{~mm}$.

a)
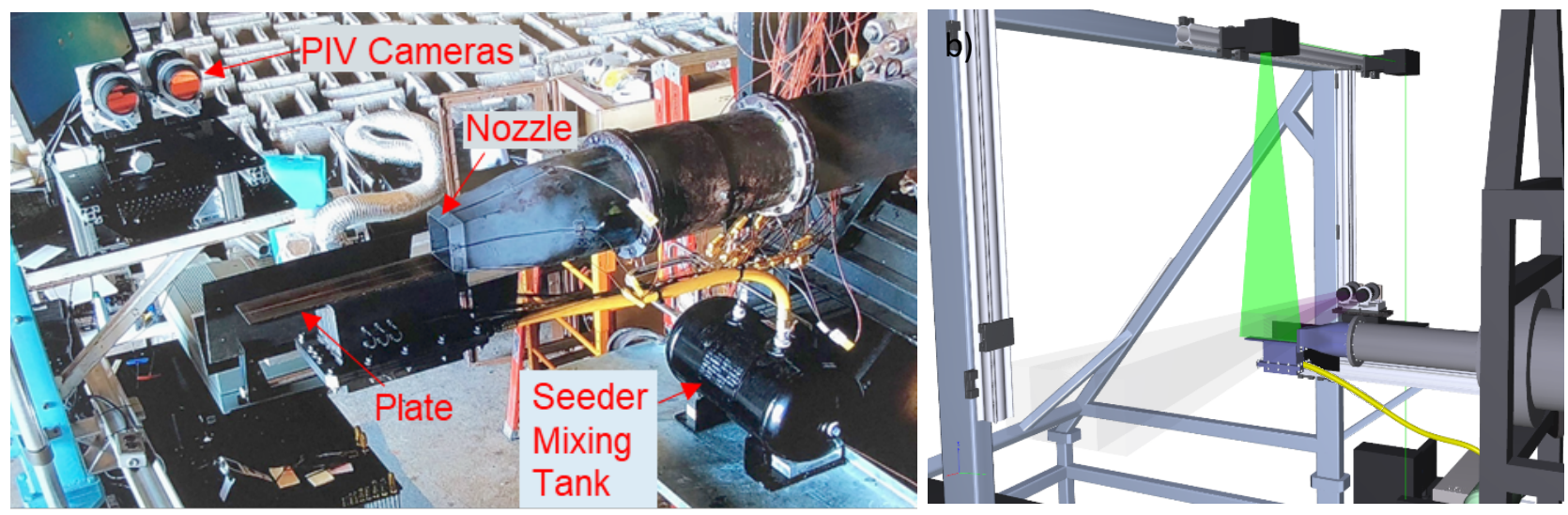

Figure 10. a) Streamwise 2-component PIV installation on the SHJAR showing dual side-by-side cameras and seeder mixing tank; b) the CAD rendering shows the laser light sheet impinging down on the centerline of the plate from above.

The PIV measurement plane was illuminated using a dual head $400 \mathrm{~mJ} /$ pulse Nd:YAG laser system. For the SPIV system, the laser beams were formed into a $1 \mathrm{~mm}$ by $100 \mathrm{~mm}$ high light sheet using cylindrical and spherical lenses, see Fig. 9. The light sheets were directed horizontally across the plate, parallel to the surface of the plate. The axial locations measured were at 12.7, 36.1, 45.7, 55.4, 76.8, 98.3, 107.9, 117.6, 139.1, 160.5, 170.2, 179.8, 208.0, and $270.3 \mathrm{~mm}$ from the exit plane of the nozzle, as depicted in Fig. 11 for a sample case collected in these experiments and also shown in Fig. 4. For the streamwise PIV measurements, the laser light sheet was expanded into a $1 \mathrm{~mm} \times 330 \mathrm{~mm}$ wide sheet from overhead and was directed down onto the centerline of the plate, see Fig. 10b. A blocking plate was placed between the cameras and the perforated plate in order to block flare light from the laser sheet from reaching the cameras.

\subsection{PIV Vector Processing}

Velocity vector maps for each camera were computed from the image pairs using NASA Glenn in-house PIVPROC software, [Wernet, 2003]. The software utilizes conventional multi-pass PIV cross-correlation processing algorithms and incorporates error detection based on image correlation signal to noise ratio. First-pass interrogation region sizes of $64 \times 64$ pixels on 32 pixel centers and final-pass interrogation region sizes of $32 \times 32$ pixels on 8 pixel centers were used to process image pairs from the stereo PIV configuration. Symmetric Phase Only Filtering (SPOF) was also employed to reduce the effects of flare light on the nozzle models directly behind the measurement planes [Wernet, 2005]. Without the SPOF processing, images with the nozzle illuminated by flare light behind the plane of interest generally produce regions in and around the potential core flow with invalid vector measurements. The SPOF processing technique was not utilized with the axial-flow measurement planes as the nozzle does not appear in the field of view. Final-pass subregions of $16 \times 16$ pixels on 8 pixel centers were used to process the streamwise PIV data. For both the 2C and Stereo PIV setups, sequences of 400 velocity vector maps were acquired at each measurement station. The image sequences were ensemble averaged to provide first- and second-order statistics over the entire measurement plane. Chauvenet's criterion was used to eliminate any outliers in the ensemble averaging process [Taylor, 1982]. The final streamwise 2C PIV velocity vector maps had a spatial resolution of $0.39 \mathrm{~mm}$, while the final cross-stream SPIV velocity vector maps had 


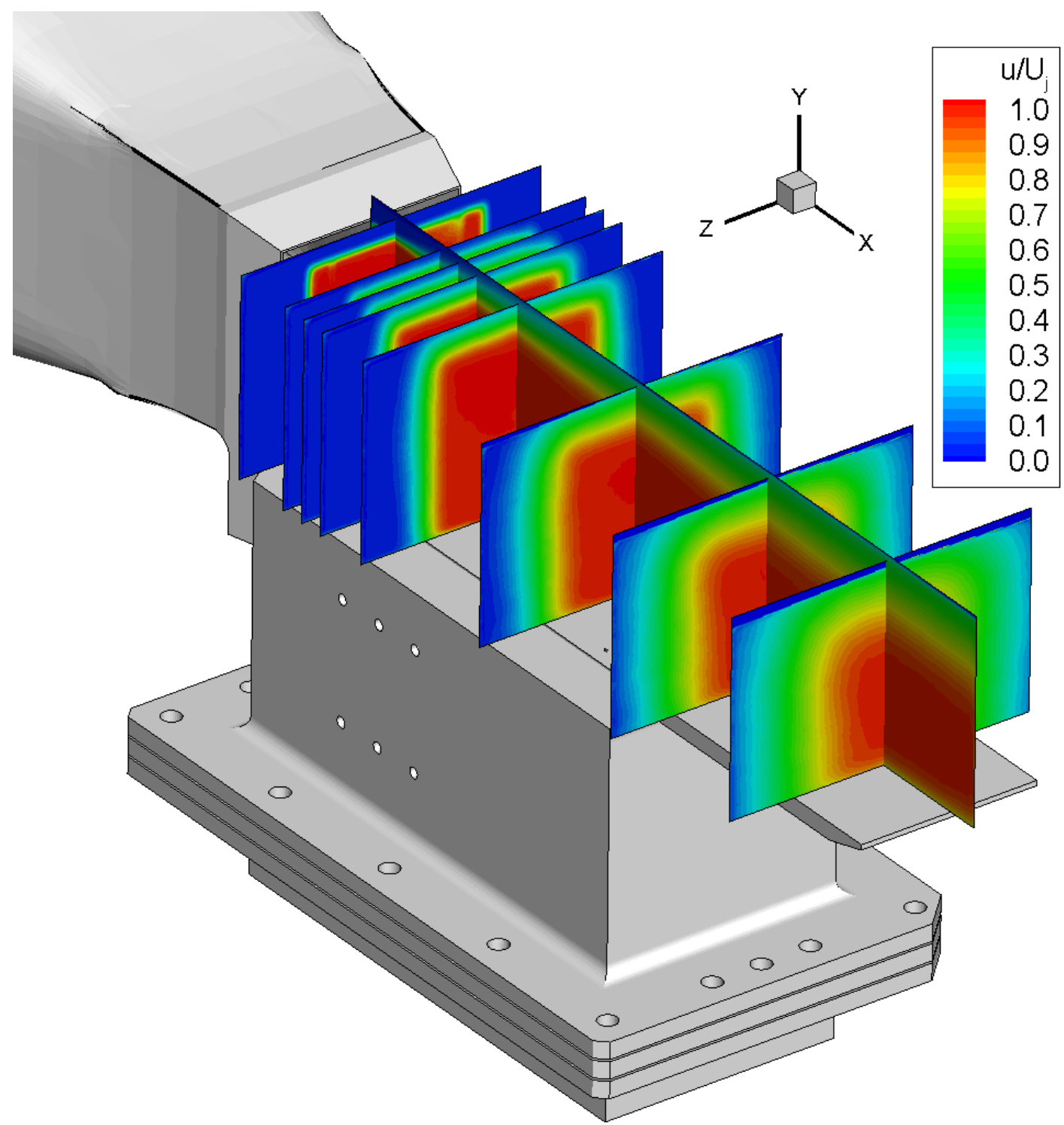

Figure 11. Axial velocity component $\mathrm{u} / \mathrm{U}_{\mathrm{j}}$ in the centerline streamwise PIV plane along with 14 cross-stream planes acquired using stereo PIV.

$0.35 \mathrm{~mm}$ spatial resolution. For the $3 \mathrm{C}$ SPIV data, the left/right vector maps were processed with an additional inhouse code to generate the 3 -D vector maps. The $2 \mathrm{C}$ streamwise PIV system provides the $\omega_{z}$ component of vorticity and the 3 C SPIV data provides the $\omega_{\mathrm{x}}$ component of vorticity. The processed PIV data for both PIV configurations have a full scale measurement error of $1 \%$.

\subsection{Flow Seeding}

For any type of PIV measurements, the fluid motion being measured is marked by the use of small particles. These particles must be sufficiently small so they will have minimal or no slip relative to the fluid (so that their motion is the same as the fluid motion). In addition, all of the fluid must be laden with particles at a concentration high enough that sufficient particles (5-10) are found in an interrogation region of the recorded PIV images. In tests using the SHJAR, three fluid streams are being mixed: the core heated square nozzle stream, the injected cooling air, and the ambient air. It is also crucial that the seed be fully mixed and dispersed in the flow upstream of the measurement region in order to ensure good-quality PIV images. Finally, the seed must not be affected by the high temperatures of the gas; this is especially true of the seed in the $800 \mathrm{~K}$ nozzle flow, for the Set Point 49 case.

The hot nozzle flow described above was seeded with a refractory seed material, and the ambient air was seeded using a commercial smoke generator. The refractory seed material used for the heated jet flow was $0.4 \mu \mathrm{m}$ diameter alumina powder. A dispersion of the alumina seed material in $100 \%$ ethanol was prepared using a $\mathrm{pH}$ stabilization technique 
[Wernet and Hadley, 2016]. The alumina/ethanol dispersion was introduced into the flow well upstream of the nozzle using an air-assisted atomizing nozzle. The $\mathrm{pH}$ stabilization technique provides highly dispersed, unagglomerated seed particles in the flow. The injected cooling air stream was also seeded using alumina, via a fluidized bed seeder system. The injected air was premixed with the dry seed material in a plenum chamber before entering the lower plenum on the model plate. A concrete vibrator was used to vibrate the dry fluidized bed seeder to help entrain seed particles into the cooling flow stream. The total mass flow of injected air was measured, upstream of the seeder, so that the blowing ratio could be accurately set. The ambient fluid was seeded with $0.3-\mu \mathrm{m}$ mineral oil droplets $\left(\rho=0.84 \mathrm{gm} / \mathrm{cm}^{3}\right)$ produced by a commercial 'smoke' generator. A pair of $1-\mathrm{m}$ diameter room circulation fans was used to disperse the concentrated smoke emitted by the smoke generator, providing a low velocity $(1 \mathrm{~m} / \mathrm{s})$, uniformly seeded ambient air around the research jet.

Although not reported here, some PIV data were acquired during the THX IV test program at supersonic conditions. The flow following fidelity of the particles is important in all PIV studies and especially in a supersonic flow investigation. The relaxation time of the alumina particles was computed to be $1.96 \mu$ s using the process outlined in Melling 1997. Similarly, the relaxation time for the oil droplets used to seed the ambient flow was determined to be $0.1 \mu \mathrm{s}$, which is significantly less than the alumina particles. Assuming Stokes drag law for a sphere, a numerical integration was then performed to compute the alumina particle relaxation distance to a step change in velocity across a shock. The distance for the alumina particles to reach $87 \%$ of the flow velocity was computed, yielding a relaxation distance of $2 \mathrm{~mm}$. The PIV subregions used to process the data were on the order of $2 \mathrm{~mm}$. Hence, the alumina particle relaxation in the jet core is masked by the spatial averaging caused by the subregions. The particle relaxation occurs over 1-2 subregions, which results in minimal smearing of the flow features for the data shown here.

\subsection{Real-Time Background Oriented Schlieren}

Background Oriented Schlieren (BOS) is a widely used technique for measuring density gradients in fluid flows of interest. The attraction of BOS is the simplicity in the setup and data reduction. In this work two innovations to the BOS technique were employed. First, a GPU based computer was used to acquire and process the BOS image data in realtime, providing a live display of the density gradients in the flow [Wernet, 2019]. Secondly, an innovative approach for generating the background speckle patterns was employed. The speckle patterns required for the BOS measurement were displayed on a high definition $4 \mathrm{~K}$ resolution computer monitor. Use of the $4 \mathrm{~K}$ monitor to display the speckle patterns has three distinct advantages: 1) the speckle patterns can be generated on the computer and displayed directly on the monitor without having to physically construct the speckle pattern; 2) the scale of the speckle pattern can be readily changed to optimize the BOS system performance; and 3) the speckle pattern is self-illuminating, which greatly simplifies implementing the technique in confined environments, or where illumination of a static speckle pattern may be difficult or problematic. A $2.4 \times 2 \mathrm{~K}$ pixel, GigE interface camera was used to image the speckle pattern displayed on a $1092 \mathrm{~mm}$ (43") diagonal $4 \mathrm{~K}$ resolution monitor. The camera was mounted on top of the large traverse system and the monitor was located on the floor of the facility, as shown in Fig. 12. The size and density of the speckle pattern selected for display on the $4 \mathrm{~K}$ monitor was determined before the test using an optimization software package. The configured Real-Time BOS system runs through an optimization algorithm which cycles through a range of speckle patterns displayed on the $4 \mathrm{~K}$ monitor while monitoring the background noise in the processed BOS images. The optimization process yields the maximum sensitivity in the configured BOS system. The processed density gradient maps were processed at the maximum $12 \mathrm{~Hz}$ frame rate of the 5MP GigE camera.

\subsection{Infrared (IR) Camera Measurements}

Temperature measurements of the plate surface were obtained using an infrared (IR) thermography technique. The surface of the plate was not painted in order to avoid any changes in the surface roughness and/or uniformity during different portions of the test program where other optical diagnostics may require direct impingement of high energy laser beams/sheets onto the pate surface. A FLIR model SC655 camera (640x480 pixels) was used to acquire infrared images of the test surface across the full range of the test matrix in Table 1 . The camera was mounted approximately $1.7 \mathrm{~m}$ from the test plate and oriented just slightly off the normal to the plate to avoid image reflections. The plate spanned approximately 100x 500 pixels on the IR camera sensor, yielding a scale factor of $0.61 \mathrm{~mm} / \mathrm{pixel}$. Ten images were acquired over a 5 second period at each Set Point. No noticeable change in the plate temperature was observed across the sequence of images. The center image in each sequence (image \#5) was used for the analysis of surface temperature. Temperature conversions of the IR images were obtained using the FLIR ResearchIR software.

The IR camera images were adjusted for thermal growth of the plate across the range of Set Points. The emissivity of unpainted (dull) stainless steel is typically reported as 0.2 ; this was used as the original estimate of the emissivity for the current model. Emissivity is a function of temperature for most materials and can vary significantly at the high temperatures employed in this test. An estimate of the surface emissivity was obtained by using the thermocouple 


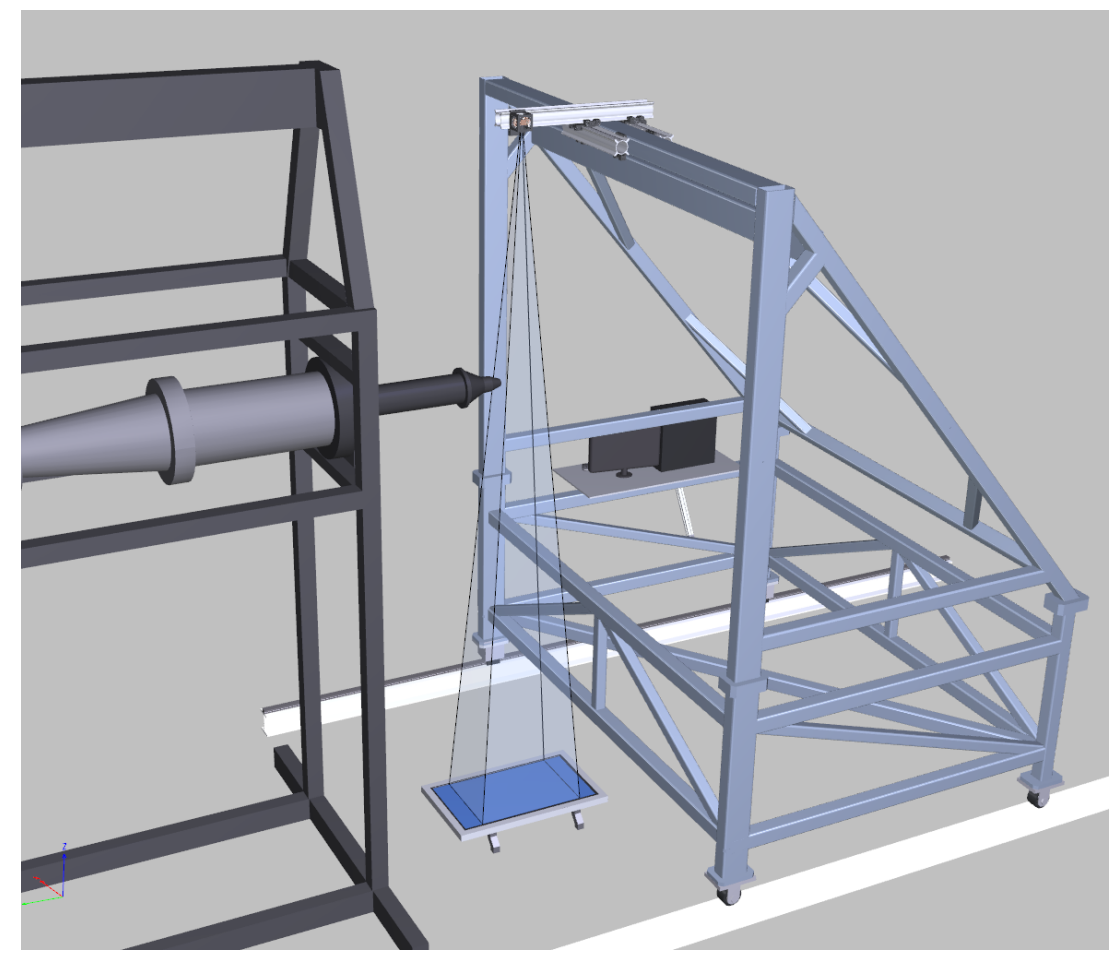

Figure 12. Background Oriented Schlieren (BOS) system installation in the SHJAR.

readings at stations 3,5 and 6 , which correspond to just before the second patch of injector holes, just before the third patch of injector holes, and after the third patch of injector holes, respectively (See Fig. 4). A calibration of emissivity with respect to temperature was determined by using surface thermocouples and the IR temperature estimates at corresponding locations on the plate. An average emissivity for the entire plate was estimated using a least squares fit to the 3-temperature thermocouple average. The calibrated emissivity values were then used to compute the temperature across the plate for each Set Point acquired. The uncertainty of the thermocouple measurements was estimated at 2\%; the uncertainty of the infrared temperature was determined to be $3.5 \%$, most of which was due to the uncertainty in emissivity.

\subsection{Results and Discussion}

The set of operating points for which comprehensive data was collected using all of the measurement techniques, is shown in Table 1. The Set Point numbers in Table 1 correspond to quantities frequently used in previous AAPL/SHJAR nozzle/jet tests, based on the jet acoustic Mach number and jet exit static temperature ratios. Set Points 23 and 42 both correspond to $\mathrm{M}_{\mathrm{a}}=0.5$, while set point 49 corresponds to $\mathrm{M}_{\mathrm{a}}=0.9$. The other columns are as follows: $\mathrm{TR}=$ jet static temperature ratio (fully expanded jet static temperature divided by the ambient static temperature); NPR = nozzle pressure ratio (primary nozzle total pressure divided by ambient static pressure); $\mathrm{M}_{\mathrm{j}}=$ jet exit fully expanded Mach number (assuming the ratio of specific heats $=1.4$ ); and $\mathrm{BR}=$ blowing ratio. As described earlier in this paper, $\mathrm{BR}$ was defined in these experiments based on the area-averaged $(\rho u)_{i n j}$ and $(\rho u)_{j}$, or $B R=\left(m_{i n j} / m_{j}\right)\left(A_{j} / A_{i n j}\right)$. For the nozzle flow, with discharge coefficient typically 0.98 or higher, the flow properties would be uniform over most of the exit plane. Hence, the area-averaged ( $\rho \mathrm{u})$ was very close to the value at the center of the jet at the nozzle exit. However, for the cooling flow, there may have been non-negligible variation among the 135 different cooling holes. Further, the cooling holes had length-to-diameter ratios of 12.5 , therefore across any hole exit, the velocity profiles were certainly far from uniform. Consequently, $(\rho \mathrm{u})_{\text {inj }}$ as defined here is the area averaged total injector flow (measured by a mass flow meter upstream of the dual-plenum system), with the total area calculated using the total exit area of the 135 cooling holes. 
Table 1. Operating Set Points with operating conditions.

\begin{tabular}{|c|c|c|c|c|c|c|}
\hline Set Point & TR & NPR & $\mathbf{M}_{\mathbf{j}}$ & $\begin{array}{c}\text { Blowing } \\
\text { Ratio } \\
\text { (BR) }\end{array}$ & $\begin{array}{c}\text { Nozzle } \\
\text { Mass Flow } \\
\text { Rate [kg/s] }\end{array}$ & $\begin{array}{c}\text { Injector Mass } \\
\text { Flow Rate } \\
{[\mathbf{k g} / \mathbf{s}]}\end{array}$ \\
\hline 23 & 1.765 & 1.103 & 0.376 & 0.0 & 0.53 & 0.0 \\
\hline 23 & 1.765 & 1.103 & 0.376 & 0.5 & 0.53 & 0.0062 \\
\hline 23 & 1.765 & 1.103 & 0.376 & 1.0 & 0.53 & 0.0124 \\
\hline 23 & 1.765 & 1.103 & 0.376 & 2.0 & 0.53 & 0.0249 \\
\hline 42 & 2.7 & 1.066 & 0.304 & 0.0 & 0.34 & 0.0 \\
\hline 42 & 2.7 & 1.066 & 0.304 & 1.0 & 0.34 & 0.0081 \\
\hline 42 & 2.7 & 1.066 & 0.304 & 2.0 & 0.34 & 0.0162 \\
\hline 42 & 2.7 & 1.066 & 0.304 & 3.0 & 0.34 & 0.0241 \\
\hline 49 & 2.7 & 1.692 & 0.900 & 0.0 & 1.02 & 0.0 \\
\hline 49 & 2.7 & 1.692 & 0.900 & 0.5 & 1.02 & 0.0121 \\
\hline 49 & 2.7 & 1.692 & 0.900 & 1.0 & 1.02 & 0.0240 \\
\hline
\end{tabular}

Recall that for the Raman measurements, the entire square nozzle/plate assembly was rotated 90 degrees clockwise, looking upstream as shown in Fig. 8. Before Raman or PIV measurements were collected, both BOS and IR camera measurements of the cooling flow over the plate were acquired. The processed BOS measurements were used to determine the $\mathrm{Y}, \mathrm{Z}$ locations of the Raman measurement points above the plate. Because the number of Raman temperature measurement locations was time-limited, these BOS images were very helpful in setting these locations. Examples of the BOS results are shown in Fig. 13 as color contours of the density gradient magnitude, where for Set Point 23 a no-cooling flow case $(\mathrm{BR}=0)$ is compared to a $\mathrm{BR}=2$ cooling flow. Without cooling flow, there is no observable density gradient above the plate. However, with the cooling flow turned on, the penetration and extent of the cooling flow above the plate is readily observed. There is an initial "hump" in the cooling film after the first patch of holes which starts to thin down just before the $2^{\text {nd }}$ patch of cooling holes (red region of high density gradient), where again the film layer thickens and steadily grows for the remainder of the plate. The BOS measurements provided a clear picture of the extent of the cooling film flow above the plate and facilitated the optimization of the $\mathrm{Y}, \mathrm{Z}$ coordinates for the Raman measurement grid. The measurement grids for each Set Point are in general not identical, even for the same blowing ratio. Raman measurements were acquired at 11 axial locations $(X=12.7,36.1,55.4,76.8,98.3,117.6,139.1$, $160.5,179.8,208.3$ and $270.3 \mathrm{~mm}$ ), which is a subset of the 14 axial locations where cross-stream PIV was taken along the nozzle centerline $(\mathrm{Z}=0 \mathrm{~mm})$. The Raman measurement grid is shown in Fig. 4 at the intersection of the green vertical lines with the green centerline. Only two of the Raman axial measurement locations coincided with the thermocouple measurement locations. Due to the time required to collect Raman data at each spatial point (approximately 3-5 minutes), the total number of Raman measurement locations was limited to approximately 140 for a given run day. At each downstream axial location, nominally 10 measurement points were acquired. The spacing of points was geometrically expanded and adjusted to cover the expected cooling film penetration into the freestream. A sample measurement grid for Set Point 23 is shown in Fig. 14; note that tightly spaced points near the surface of the plate are not clearly discernable. At the beginning of a run day, spectra were recorded to provide a background spectra for baseline removal. Additionally, Rayleigh line spectra were acquired (by removing the RazorEdge long-pass filter) each run day for characterization of the instrument function. At each measurement station, 1000 single shot spectra were recorded. The data were processed as previously described, ensemble averaged, and stored in Tecplot compatible file formats.

Temperature data were acquired over a wide range of flow temperatures and Mach numbers as listed in Table 1. In this paper, results for two of these conditions are presented: Set Points 23 and 49, which are at $\mathrm{M}_{\mathrm{j}}=0.376$ and 0.9 flows with temperature ratios of 1.77 and 2.7, respectively. An ambient temperature point was acquired prior to the start of each run day using the Raman measurement system. These ambient temperatures and their rms temperature variations provide a check on the performance of the Raman measurements in the jet flow facility. 

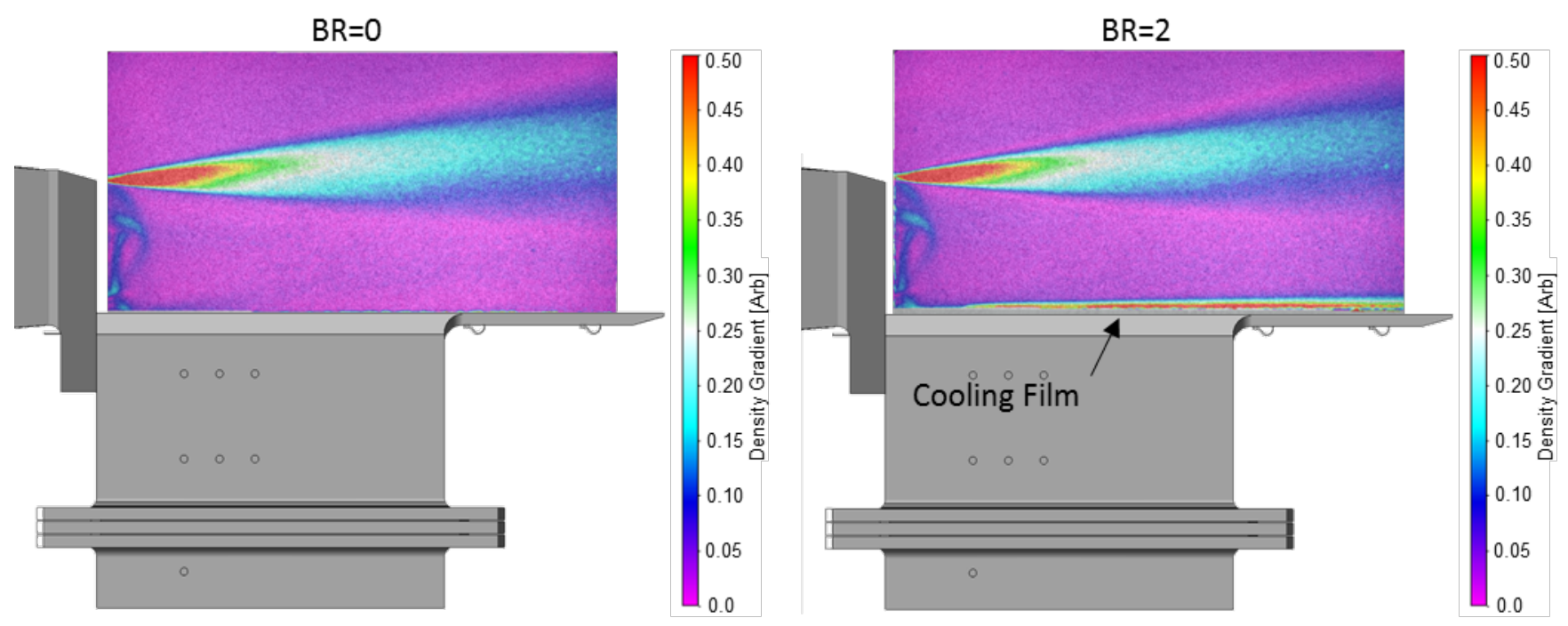

Figure 13. BOS density gradient maps for Set Point 23: a) $B R=0$ and b) $B R=2$.

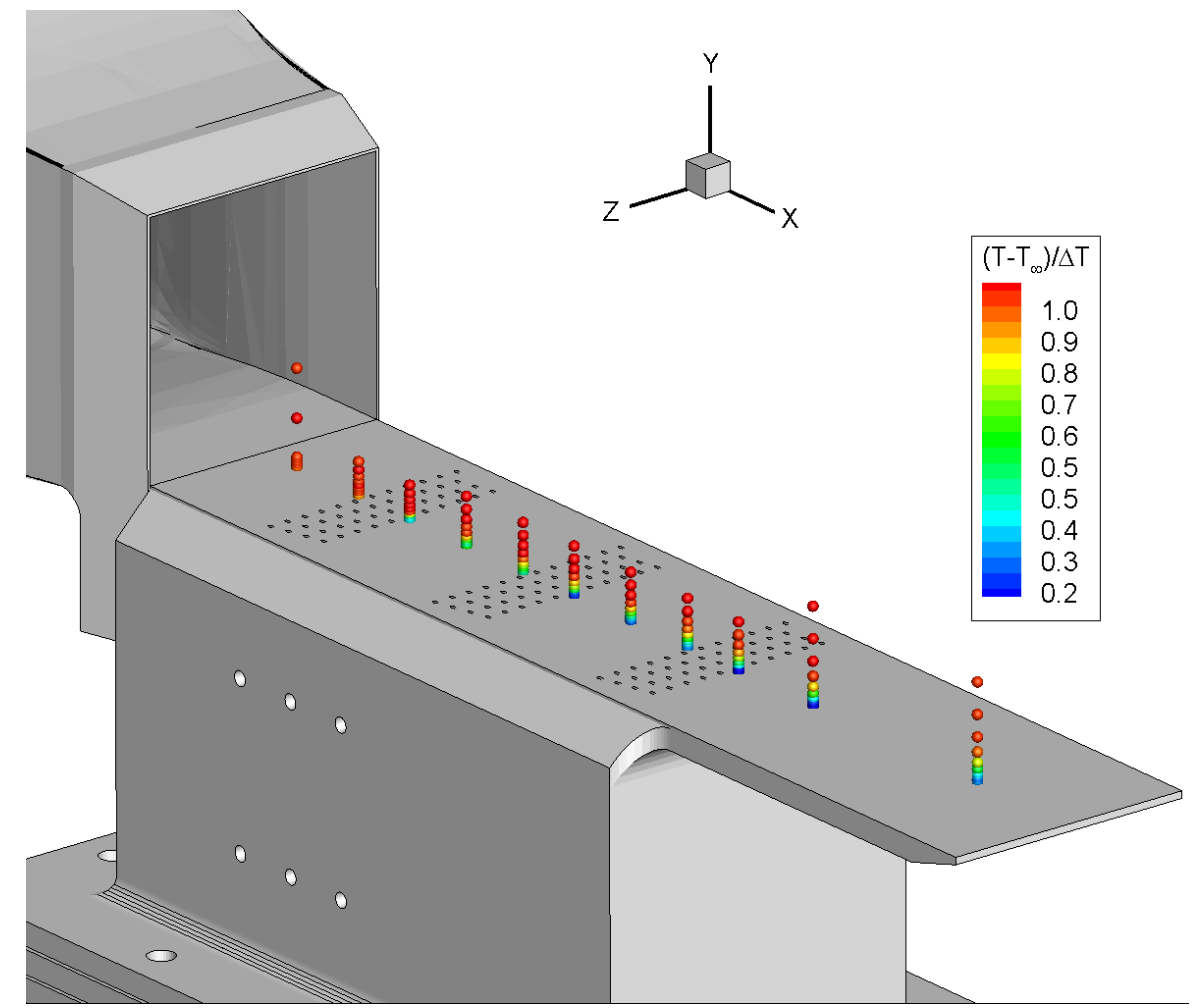

Figure 14. Nominal measurement grid and an example of Raman measured temperatures for Set Point 23, BR=2.0.

Both streamwise and cross-stream PIV data were collected across all of the Set Points listed in Table 1. Cross-stream PIV data were collected at the same locations as the Raman temperature measurements, as shown in Fig. 4 by the solid vertical green lines, and at 3 additional planes located in the centers of the cooling hole patches denoted by the vertical dashed lines. The cross-stream PIV data at 14 planes were processed and merged into a single block of data and stored in Tecplot compatible file format. As mentioned in the data processing section, obtaining cross-plane vector maps without contamination from the model in the background is very difficult. The issue is exacerbated by the alumina seed material which coats the inside surfaces of the square nozzle and plate surface over time making them white and brighter in the background of the illuminated PIV measurement planes. The presence of the vertical sidewalls of the nozzle in the background results in regions of low signal to noise in the images, which causes spurious vectors in the processed vector maps. The 3D block of cross-stream PIV data enables the data to be sliced across any plane. The cross-stream PIV data reveal the complex flow features of the injected thin film cooling flow down near the plate surface. A series of X-Z plane 
slices through the data set at increasing Y-heights above the plate are shown in Fig. 15. The plots in Fig. 15 show velocity vectors at 13 of the 14 measured planes along the plate for Set Point 23 at a BR $=2.0$. Cross-stream PIV measurements were acquired $1 \mathrm{~mm}$ upstream from each patch of cooling holes, in the center of patch of cooling holes and $1 \mathrm{~mm}$ downstream of each patch of cooling holes. The vectors in Fig. 15a are colored by the v-component (vertical) of velocity normalized by the jet exit velocity: $\mathrm{v} / \mathrm{U}_{\mathrm{j}}$. The laser light sheet propagates from left to right and the left camera sees more of the forward scattered light off of the model than the right camera in the PIV stereo camera pair. As the PIV system is traversed downstream the location of the flare light in the image marches to the left. The black triangle in Fig. 15a denotes the region of the reconstructed 3D flow field that is contaminated by the flare light off of the square nozzle in the background of the PIV data acquisition planes. The contamination is worse near the plate surface and at the top of the nozzle where the corners of the square nozzle reflect/scatter the most light from the laser sheet and from secondary scatter from seed particles in the flow. Flow features inside the black triangle are not considered accurate. In Fig. 15a, (Y= $0.52 \mathrm{~mm})$ the cooling film from the first patch of cooling holes, $(X=45 \mathrm{~mm})$ penetrates up into the hot freestream flow. The sinusoidal profile of the velocity vector tips to the right of the black triangle reveals that the vertical velocity component of the injected cooling flow is higher than that of the freestream flow. In the second patch, the injected cooling flow has a stronger vertical component than in the first cooling hole patch. The cooling holes at the edges of the $2^{\text {nd }}$ patch, which are in the heated nozzle flow shear layer, have an even larger vertical component. The cooling flow in the potential core of the jet is suppressed by the dynamic pressure of the flow in the jet core compared to the turbulent flow in the shear layer. In the $3^{\text {rd }}$ row of cooling holes the collapse of the potential core is more pronounced. Fig. $15 \mathrm{~b}$ shows an X-Z plane slice of vectors colored by $\mathrm{v} / \mathrm{U}_{\mathrm{j}}$ at $1.52 \mathrm{~mm}$ above the plate surface. The individual jets in the $1^{\text {st }}$ patch of cooling holes is still visible. The flow between cooling hole patches is relatively horizontal. The individual jets from the $2^{\text {nd }}$ and $3^{\text {rd }}$ cooling hole patches are barely discernable, however the cooling film has a definite uniform vertical velocity across the cooling hole patch, indicating that the cooling film is growing in height. Fig. 15c shows an X-Z plane slice of vectors colored by $\mathrm{u} / \mathrm{U}_{\mathrm{j}}$ at $\mathrm{Y}=21.8 \mathrm{~mm}$, approximately at the centerline of the square nozzle. The flow over the plate is uncontaminated by flare light at the nozzle centerline as illustrated by the uniform velocity profiles. The thickness of the shear layer and the decreased width of the potential core are readily observed.
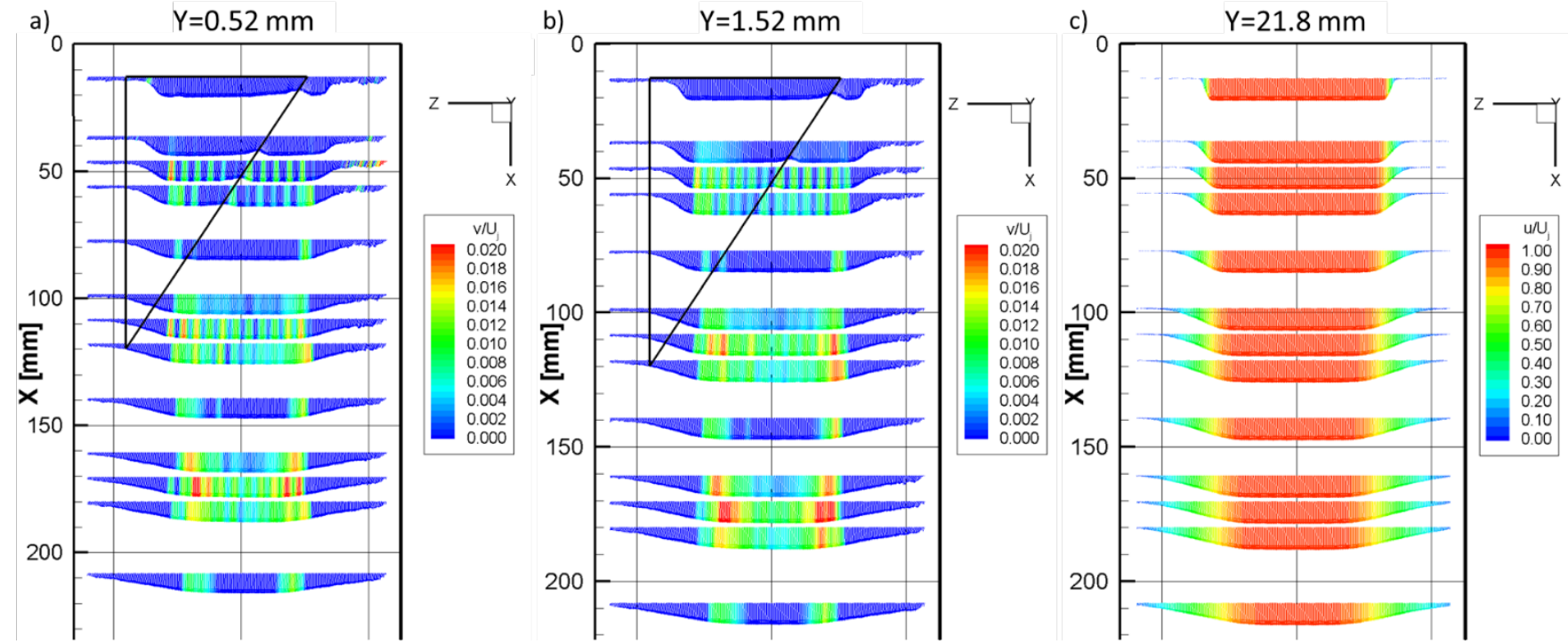

Figure 15. X-Z plane vector plots of the Cross-stream PIV data at different Y-heights above the plate for Set Point 23, $\mathrm{BR}=2$. For $15 \mathrm{a} \& \mathrm{~b}$, the vectors are colored by the normalized v-component $\left(\mathrm{v} / \mathrm{U}_{\mathrm{j}}\right)$ of velocity and in $15 \mathrm{c}$ the vectors are colored by $\mathrm{u} / \mathrm{U}_{\mathrm{j}}$.

While always a challenge, seeding the ambient flow at the appropriate level proved to be difficult during the cross-stream PIV section of the test. The ambient seeding levels were nominally too high for the Set Point 42 and Set Point 49 cases. The Set Point 49 cross-stream PIV data have a distinctly higher noise level than the other two Set Point conditions due to difficulties in obtaining optimal flow seeding.

The streamwise PIV data were collected using an overhead light sheet, which impinged on the plate as depicted by the green line down the centerline of the plate in Fig. 4. Flare light from the plate surface was blocked from reaching the cameras using a plate placed parallel with the surface of the plate, located between the PIV cameras and the model. The light sheet blocking plate was raised just high enough to attenuate the flare light from the $304.8 \mathrm{~mm}$ long plate surface. Again, as in the cross-stream PIV data, the alumina seed material eventually covered the surfaces of the model with a white coating, increasing the amount of flare light being scattered. The amount of seed buildup on the plate surface varied 
depending on the blowing ratio of the cooling film. A sample image of the seed coated plate surface during the streamwise PIV testing is shown in Fig. 16. The after-image of the laser light sheet impinging on the centerline of the plate is observed down the centerline of the plate. Seed material accumulated on the plate surface downstream of the cooling injection patches. Briefly increasing the cooling air blowing ratio typically removed most of the seed buildup from the plate. However, during acquisition of the PIV image data some buildup of the seed on the plate was occurring, yielding both increased flare light from the surface and changes to the surface roughness and potentially the flow over the plate.

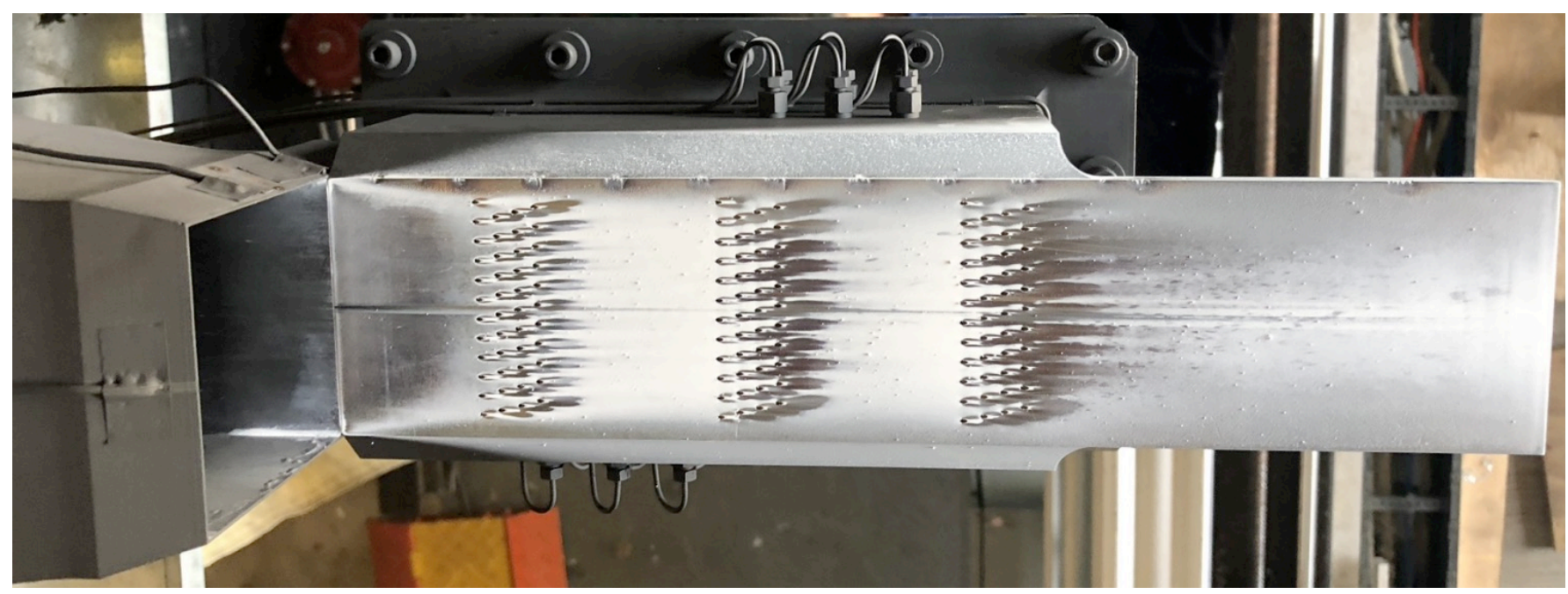

Figure 16. Image of the plate after a streamwise PIV data acquisition series.

\subsection{Set Point 23 PIV And Raman Results:}

The Set Point 23 streamwise PIV data are plotted as line profiles of u normalized by the jet exit velocity $U_{j}$ across the full height of the square nozzle in Fig. 17. Line profiles along the plate centerline are extracted at the same axial stations where Raman data were collected. The line plots clearly illustrate the growth of the shear layer and the collapse of the core region of the flow as the top shear layer thickens. For the $\mathrm{BR}=0$ case, there is steady boundary layer growth with increasing distance down the plate. With the blowing turned on at $\mathrm{BR}=1$, the free shear layer side of the profiles remains relatively unchanged; however, there is a significant change in the velocity profiles near the plate where the transition from the injected air at the plate mixing with the hot freestream flow extends up higher above the plate with increasing distance down the plate.
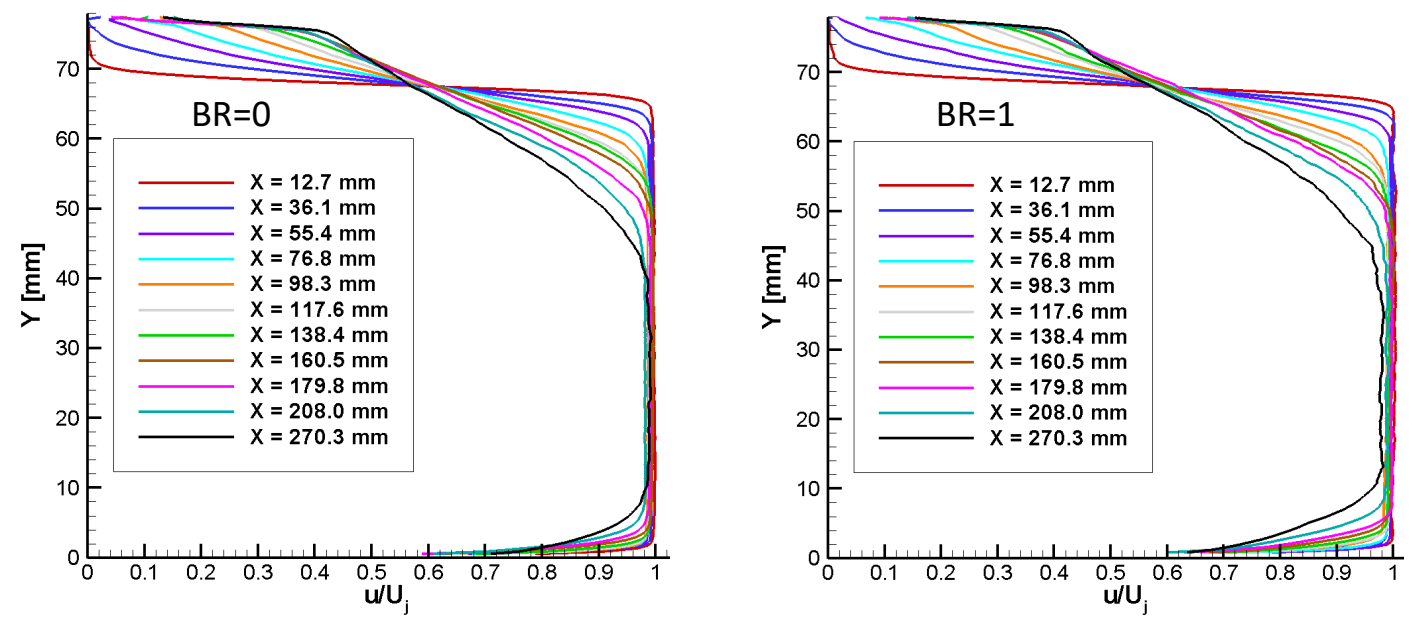

Figure 17. Full height velocity profiles of the Set Point 23: a) $B R=0.0, b) B R=1.0$ cases on the plate centerline.

Figure 18 shows a close up view of the streamwise PIV line profiles of both mean and rms velocities. Again, the line profiles correspond to the axial stations where both PIV velocity and Raman temperature data were collected. Note that there are three additional cross-stream PIV planes, located in the center of each cooling hole patch, that are not shown in Fig. 18. The color key at the bottom of the plot depicts where each line profile was acquired along the plate. At $\mathrm{BR}=0$, the boundary layer growth is minimal until after the $2^{\text {nd }}$ patch of holes at $117.6 \mathrm{~mm}$ down the plate. At $\mathrm{X}=138.4 \mathrm{~mm}$ and beyond, a steady increase in the thickness of the velocity boundary layer is observed. Although rms velocity 
measurements are not made very close to the wall where the shear is highest and the rms quantities will peak, the behavior of the rms profiles in the outer portion of the boundary layer shows a steady increase in both thickness of the shear layer and a steady rise in the turbulence in the flow away from the plate with increasing distance down the plate. Note that this turbulence is due to the end of the potential core of the hot jet. At $\mathrm{BR}=1$, the boundary layer thickness is fairly constant $(\mathrm{Y}<2 \mathrm{~mm}$ ) up to $\mathrm{X}=76.8 \mathrm{~mm}$, after which the boundary layer thickness increases due to both normal boundary layer growth of the heated stream and insertion of the lower velocity injected film cooling air, which then mixes with the freestream heated flow. The rms velocity profiles show that the injected film layer does not develop significantly until after the $2^{\text {nd }}$ patch of cooling holes, after which the height of the shear layer significantly increases $(\mathrm{Y}>4 \mathrm{~mm})$. This may be evidence that more net cooling flow comes from the second and third patches of cooling holes, than from the first patch.

This trend does not continue at $\mathrm{BR}=2$. Instead, the velocity profiles converge much more tightly than in the no-blowing case or $\mathrm{BR}=1$ case. The blowing ratio is nominally defined as $(\rho \mathrm{U})_{\mathrm{inj}} /(\rho \mathrm{U})_{\mathrm{j}}$ and the temperature ratio for Set Point 23 is 1.765. In a pressure-matched situation, the density ratio varies as the inverse of the temperature ratio. Therefore, at a blowing ratio of $2, \mathrm{U}_{\mathrm{inj}} / \mathrm{U}_{\mathrm{j}}$ equals 1.133 . However, $\mathrm{U}_{\mathrm{inj}}$ is injected at $30^{\circ}$ to the flow. The axial component of velocity, which is plotted in Fig. 18 is $\mathrm{U}_{\text {inj }} \cos \left(30^{\circ}\right)$, which yields a $\mathrm{u} / \mathrm{U}_{\mathrm{j}}$ along the $\mathrm{X}$-axis of 0.981 . Hence, the axial velocity component of the injected film cooling layer is nearly equal to the ambient flow velocity. The boundary layer over the plate is effectively re-energized with insertion of cooling flow with axial velocity equal to the freestream velocity, resulting in similar velocity profiles near the plate. The supposition that the cooling flow velocity matches the free stream velocity is further confirmed by the significant drop in the rms values in the region that was the outer portion of the boundary layer for the $\mathrm{BR}=1$ case, for axial positions past $98 \mathrm{~mm}$, as shown in Fig. 18. For $\mathrm{BR}=2$, there is less momentum exchange happening between the cooling film and the freestream flow. 

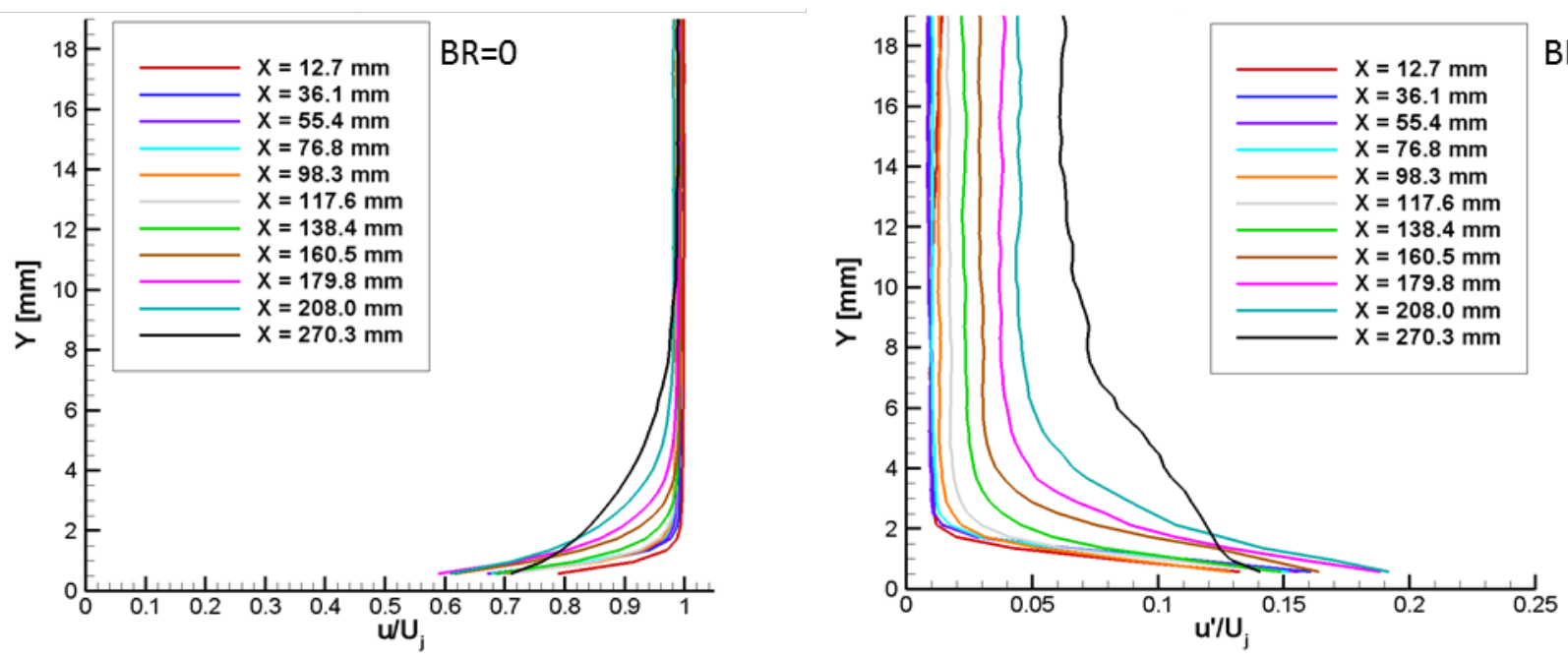

$B R=0$
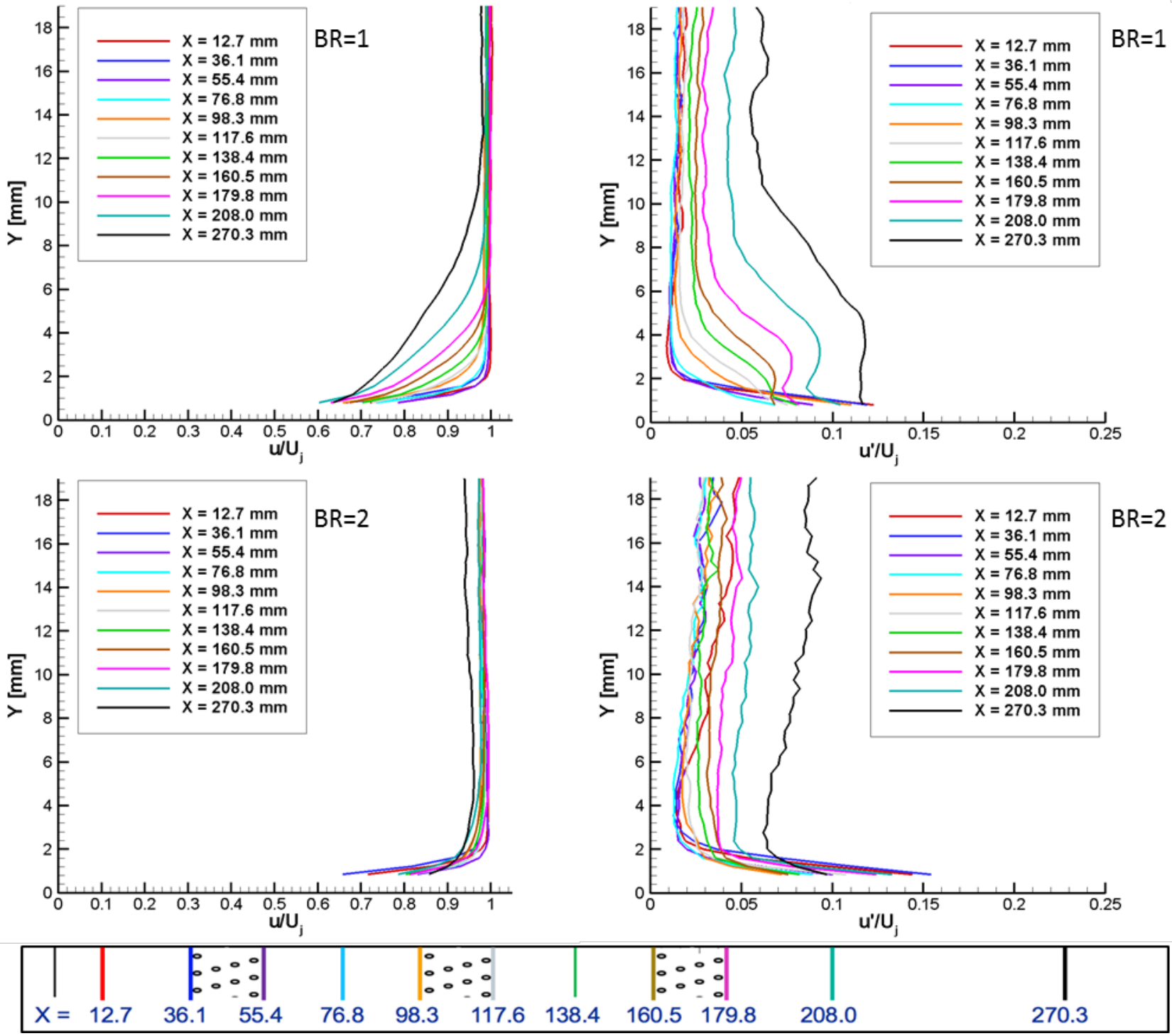

Figure 18. Streamwise PIV mean and rms velocity profiles along the centerline of the plate for Set Point 23 at $B R=0,1$ and 2. The color coded key at the bottom shows the measurement stations along the plate where the measurements were collected. 

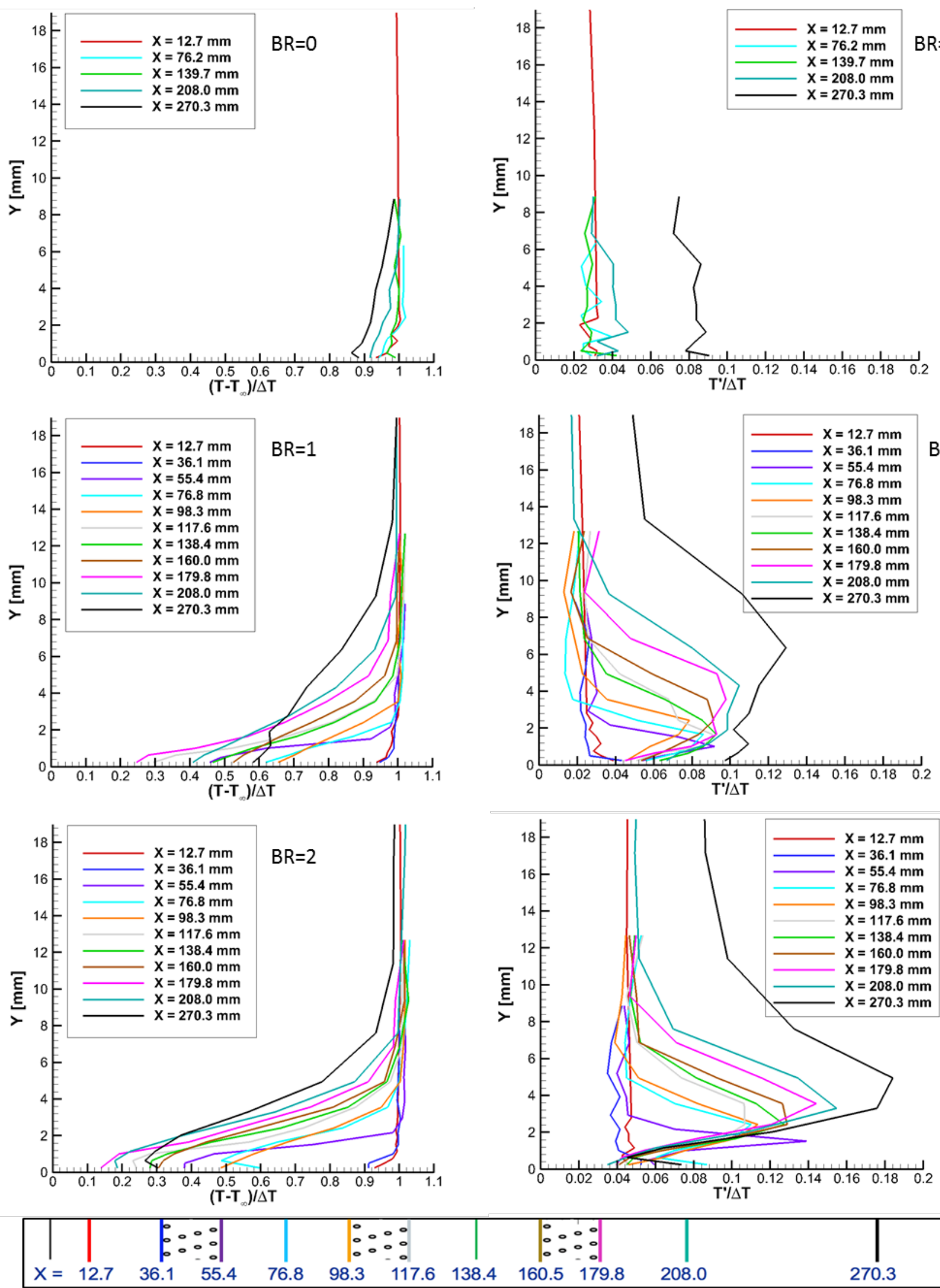

Figure 19. Raman mean and rms temperature profiles along the centerline of the plate for Set Point 23 at $\mathrm{BR}=0,1$, and 2. The color coded key at the bottom shows the measurement stations along the plate where the measurements were collected. 
The Raman temperature diagnostic data were processed for all of the grid locations across all of the Set Points listed in Table 1. In general, it is most desirable to acquire data for a single Set Point in one day so that the jet conditions and data collection calibrations are consistent. Attempts were made to acquire Raman temperature measurements as close to the plate surface as possible. While the Raman spectra acquired at $\mathrm{Y}=0.25-2.5 \mathrm{~mm}$ were dominated by the (Rayleigh/laser line) flare light off of the plate surface, the Raman temperatures were still able to be extracted from the acquired spectra. In Fig. 19, Raman temperature profiles along the centerline of the plate are presented for the three blowing ratios $(\mathrm{BR}=0$, $1,2)$ at Set Point 23. The mean temperature profiles are normalized by subtracting the ambient temperature $\mathrm{T}_{\infty}$ and then dividing by the temperature difference between the jet exit temperature and the ambient temperature $\Delta T=\left(T_{j}-T_{\infty}\right)$. The rms temperature profiles are normalized using the same $\Delta \mathrm{T}$.

For the $\mathrm{BR}=0$ case, temperature data were only acquired at 5 axial stations along the plate, since there was no film cooling. The line profiles in Fig. 19 are plotted for increasing values of the axial coordinate $\mathrm{X}$, as denoted in the legend. At $\mathrm{BR}=$ 0 , the profile at $X=12.7 \mathrm{~mm}$ indicates that a small thermal boundary layer exists above the plate, before any of the injection cooling. The heated freestream flow at $X=12.7 \mathrm{~mm}$ is observed to be relatively constant across the profile height. The normalized rms temperature at $\mathrm{BR}=0$ nominally has the same behavior as the normalized rms velocity at the jet exit, and similar to the PIV data, an increase in the rms level is observed at the furthest measurement station at $\mathrm{X}=270.3 \mathrm{~mm}$, relative to upstream.

At $\mathrm{BR}=1$, the inlet flow $(\mathrm{X}=12.7 \mathrm{~mm})$ is again observed to be uniform with a small thermal boundary layer. Immediately following the first patch of cooling holes, $X=55.4 \mathrm{~mm}$, the film cooling layer is clearly observed at $\mathrm{Y}=2 \mathrm{~mm}$ and drops sharply to a normalized temperature of 0.45 near the plate surface. At $X=55.4 \mathrm{~mm}$ the cooling film penetrates further into the flow and the mean temperature gradually increases further away from the wall, as the cooling film mixes with the free stream. The rms temperature plots at $\mathrm{BR}=1$ show the shear layer in the cooling film from the first patch of cooling holes and the increase in height of the peak shear into the freestream up to $X=98.3 \mathrm{~mm}$. Following the $2^{\text {nd }}$ cooling hole patch, $X=117.6 \mathrm{~mm}$, the cooling film penetrates further out into the flow $(\mathrm{Y}=4 \mathrm{~mm})$ and the normalized film temperature down at the plate drops to 0.28 . Further down the plate, the film penetrates further out into the freestream flow as denoted by the mean temperature profiles at $X=138.4 \mathrm{~mm}$ and $160.5 \mathrm{~mm}$. The rms temperature profiles at 117.6, 138.4 and 160.5 $\mathrm{mm}$ also show evidence of the increase in the shear layer height due to the mixing of the cooling film with the freestream. The cooling film downstream from the $3^{\text {rd }}$ cooling hole patch yields the coolest normalized temperature of 0.24 down at the plate and the highest penetration into the flow, $\mathrm{Y}=6 \mathrm{~mm}$. Again, the rms temperature profiles confirm that the film is mixing with the freestream flow and the rms levels reach a maximum for $\mathrm{X}>179.8 \mathrm{~mm}$.

For $\mathrm{BR}=2$, there appears to be a film of cool air that persists over a significant portion of the plate. The air temperatures near the plate are the lowest observed for any of the BR cases at this Set Point. Recall that all of the velocity profiles had collapsed at $\mathrm{BR}=2$ indicating that there may not be significant mean velocity shear between the freestream and the cooling film above $2 \mathrm{~mm}$ from the plate. In contrast, the mean and rms temperature profiles show that there is significant exchange of thermal energy, and that the cooling film thickness grows with increasing distance downstream. The temperature profiles near the plate have many interesting features. Following the first cooling hole patch, there is an inflection in the temperature profile $(\mathrm{X}=76.8 \mathrm{~mm}$ ) which indicates that the cooling film has lifted off of the plate and hot ambient air is mixing in underneath the lifted off cooling film. The relatively low rms values at $\mathrm{Y}=1 \mathrm{~mm}$ indicate there is little mixing of the cooling film with the ambient freestream. Just before the $2^{\text {nd }}$ patch of cooling holes, at $X=98.3 \mathrm{~mm}$, the temperature profile indicates a cool film down to the plate surface. After the $2^{\text {nd }}$ cooling hole patch, the cooling film again exhibits some concavity in the temperature profile at the plate at $\mathrm{X}=117.6,138.4$ and $160.5 \mathrm{~mm}$. The lowest film normalized temperature of 0.14 is achieved after the $3^{\text {rd }}$ cooling hole patch where initially the film appears to be attached but then may have lifted off again. Significant rms temperature fluctuations are observed above $4 \mathrm{~mm}$. The general trend observed is that the low temperature cooling film on the plate is exchanging heat with the ambient flow. Examining the collapsed mean velocity profiles, one might incorrectly assume that in the absence of mean velocity shear beyond $2 \mathrm{~mm}$ above the plate, there was no evidence of turbulence and hence no transport of heat between the cooling film and the freestream. However, it is likely that the moderate rms velocity profiles coupled with a large gradient in mean temperature, correspond to the observed high rms temperatures.

The behavior of the rms velocity and temperature profiles varies depending on the blowing ratio. However, a few profile features away from the plate are noteworthy. Above $\mathrm{Y}=12.7 \mathrm{~mm}$, the normalized rms temperature values all nominally approach the freestream rms temperature values $(2-3 \%)$; however, the rms velocity values progressively increase with increasing axial position. Upstream of the patches of cooling holes $u^{\prime} / \mathrm{U}_{\mathrm{j}}$ is around $2 \%$, as expected; however, after each successive cooling patch the freestream rms velocities steadily increase reaching $7 \%$ at $X=270 \mathrm{~mm}$. The increase in rms velocity to $7 \%$ most likely corresponds to the end of the potential core of the hot jet. The rms levels are similar to those 
expected at the end of a potential core for a simple jet exhausting into ambient. A similar trend was observed in the THX Step III PIV data [Wernet et al., 2018] where the rms velocity levels along the jet centerline increase with distance but the normalized rms temperature values approach a constant value independent of axial location.

Plotting the velocity and/or temperature profiles along the plate centerline in a single plot enables the assessment of any bulk effects such as the matching of the cooling film velocity with the heated free stream velocity. The combined profile plots also provide high-resolution details of the flow profiles, especially near the plate. However, with the complex flow features from the multiple patches of cooling film injection, the line profiles frequently overlap making interpretation of the results somewhat difficult. An alternative approach is to plot the data at the spatial locations along the plate where they were collected. The scale of the line plots will not be proportional to the scale of the model, but the flow development along the plate will be readily observed. Figures 20 and 21 show the same Set Point 23 data as shown in figures 18 and 19, except that the individual line profiles are located at the station along the plate at which they were acquired. For the mean values of temperature and velocity, the nomalized value equal to 1 is used as the common locating point in the profile relative to the measurment station. For the rms line plots, the line profiles are stationed along the plate roughly at the rms value at $18 \mathrm{~mm}$ above the plate, hence the growing offset of the rms temperatures is not preserved in these plots. A relative scale for each Set Point is shown on the plots at the last axial station. All of the observations stated above for figures 18 and 19 apply to these replotted data sets. The development of the flow profiles before and after the film cooling injection is now readily observed. The thickening boundary layer for the $\mathrm{BR}=1$ case shows the increase in the film cooling layer thickness, however, the rms velocity plots give a clearer indication of the top edge of the shear layer, and hence the film cooling layer. It is interesting to note that for the $\mathrm{BR}=2$ case, there are no significant momentum features discernable in the mean velocity profiles except that they are all very similar and would collapse on one another as shown in Fig. 18. However, there are significant thermal flow features in the film cooling flow at BR=2.0, where the cooling film lift-off is readily observed. 

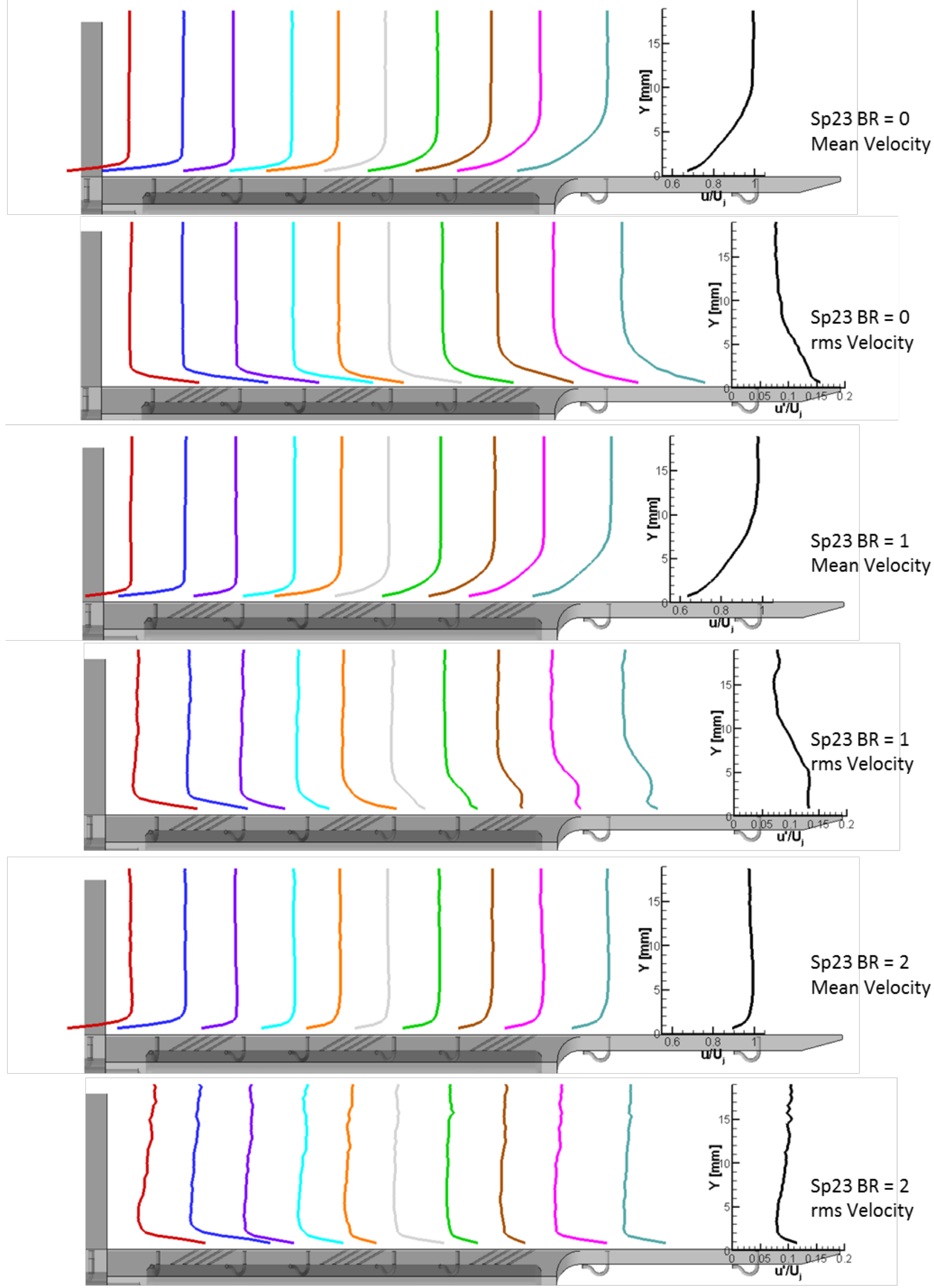

Figure 20. Streamwise PIV mean and rms velocities plotted on the model for Set Point 23, at BR =0, 1, and 2. 


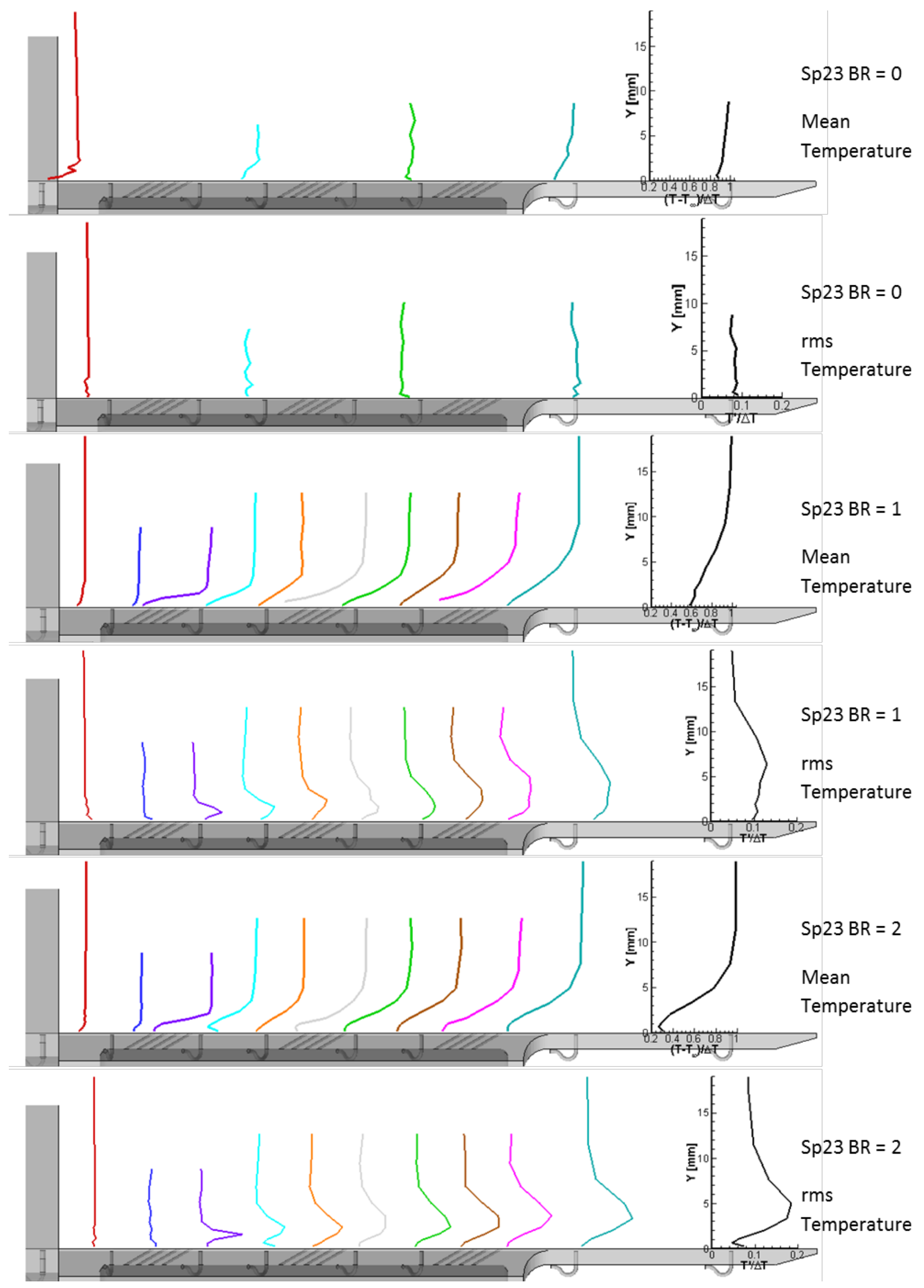

Figure 21. Raman mean and rms temperatures plotted on the model for Set Point 23, at BR =0, 1, and 2. 


\subsection{Set Point 49 PIV And Raman Results:}

The highest velocity and highest temperature conditions run during the test program were for Set Point 49 . The high velocity flow over the plate limited the maximum blowing ratio that could be set with the rig hardware, as limited by both the supply pressure and the structural burst pressure limit of the plenum. Results from the streamwise PIV for Set Point 49 at $\mathrm{BR}=0,0.5$ and 1.0 are shown again spatially distributed along the plate in Fig. 22. For the $\mathrm{BR}=0$ case the inlet flow has a thin boundary layer that grows more quickly right after each patch of cooling holes, although there is no introduction of cooling air flow into the hot boundary layer. There are three potential sources for the boundary layer growth: 1) the presence of the holes may be acting analogous to a rough surface by increasing the near wall turbulence and accelerating the boundary layer growth;2) although there is no net flow through the plenum, there could be limited unsteady "pumping" of fluid through the different cooling hole patches, analogous to a synthetic jet arrangement, which may have also amplified the turbulent growth rate; 3 ) the unavoidable buildup of seed material on the plate could have played a role in the observed boundary layer growth.

For both $\mathrm{BR}=0.5$ and $\mathrm{BR}=1.0$, the first patch of cooling flow actually seems to energize the velocity boundary layer and this results in a sharper and shorter boundary layer profile after the patch, compared to before the patch. However, for the downstream patches, the trend is that the insertion of more cooling air thickens the mean velocity profiles. For BR $=0.5$, the boundary steadily thickens, eventually growing approximately $8 \mathrm{~mm}$ thick. For $\mathrm{BR}=1.0$, the boundary layer reaches a thickness of $10 \mathrm{~mm}$. After the $3^{\text {rd }}$ cooling hole patch, the shear layer profiles show a rapid increase in mixing at the top of the cooling film and then a plateau in the shear for the last several $\mathrm{mm}$ down to the plate. The plateau in the rms velocities indicates there is little mixing occurring in this layer, and hence a preservation of the cooling film. The mean and rms velocity profiles are plotted together in Fig. 23, where the relative scale of the boundary layer development and the scale of the rms values are clearly depicted. The growth of the shear layer increases significantly at/after the $2^{\text {nd }}$ patch of cooling holes for all blowing ratios. In Figs. 24 and 25, for the BR $=1.0$ case, it appears that just before the $2^{\text {nd }}$ and $3^{\text {rd }}$ cooling hole patches, there is an inflection in the normalized temperature profiles at the plate surface. This measurement location is just upstream and centered between two cooling holes. The temperature inflection could be due to a complex interaction of the two new cooling streams and the residual cooling film from the upstream cooling patch, enabling warmer air to penetrate to the plate surface.

The Raman temperature profiles for Set Point 49 at $\mathrm{BR}=0,0.5$, and 1.0 are also plotted spatially distributed along the plate in Fig. 24. The mean and rms temperature profiles along the plate are also plotted in a combined plot in Fig. 25, where the relative scale of the boundary layer development and the scale of the rms values are clearly depicted. As in the Set Point 23 case, the $B R=0$ case shows a relatively uniform temperature profile from the exit of the nozzle which lasts the duration of the plate. A thin boundary layer gradually develops along the plate. At $\mathrm{BR}=0.5$, the cooling film presence is immediately visible after the $1^{\text {st }}$ patch of cooling holes where the temperature ratio drops to 0.6 . The cooling film mixes with the hot freestream air and turns from pseudo-parabolic to linear and penetrates higher into the freestream, up to $\mathrm{Y}=4 \mathrm{~mm}$. After the $2^{\text {nd }}$ patch of cooling holes the normalized temperature again drops to 0.6 and the temperature profile is again pseudo-parabolic, now extending up to $6 \mathrm{~mm}$ above the plate. The cooling film

temperature profile again flattens out as it mixes with the hot freestream and remains attached to the plate. This process repeats after the $3^{\text {rd }}$ patch of cooling holes where the film temperature again drops to 0.6 and then flattens out due to the mixing with the hot freestream. The cooling film is observed to steadily increase in height above the plate at $\mathrm{BR}=0.5$. The rms temperature profiles show that the main shear is occurring at the top edge of the shear layer. Further, this indicates that injected air is providing a good film cooling layer for the plate surface. For the BR $=1.0$ case, the temperature ratio drops to 0.4 just downstream of the $1^{\text {st }}$ patch of cooling holes. As the film mixes with the hot freestream flow, the temperature profile flattens out again at $X=76.8 \mathrm{~mm}$. The rms temperature profile at $X=76.8$ $\mathrm{mm}$ shows a high shear region at the top of the boundary layer and an inflection point closer to the surface. The inflection point is likely the core of the cooling film. Just before the $2^{\text {nd }}$ patch of cooling holes the mean temperature profile shows a slight inflection, which is also repeated just ahead of the $3^{\text {rd }}$ patch of cooling holes. It appears that the cooling film from the $1^{\text {st }}$ patch of holes is stagnating and possibly locally lifted-off of the surface due to the blockage created in the flow by the film entering from the $2^{\text {nd }}$ patch of cooling holes. The same flow stagnation effect is also occurring just prior to the $3^{\text {rd }}$ patch of cooling holes. The $\mathrm{BR}=1$ case exhibits the thickest cooling film, penetrating up to $10 \mathrm{~mm}$ above the plate. For $\mathrm{BR}>0$, there is a large linear temperature gradient from the freestream down to the air temperature just above the plate following each cooling hole patch. This indicates that the cooling film in both cases is attached to the plate and of sufficient mass flow to provide an efficient barrier from the hot free stream flow. The BR= 1 case provides a lower plate temperature, which may be desirable depending on the application. 

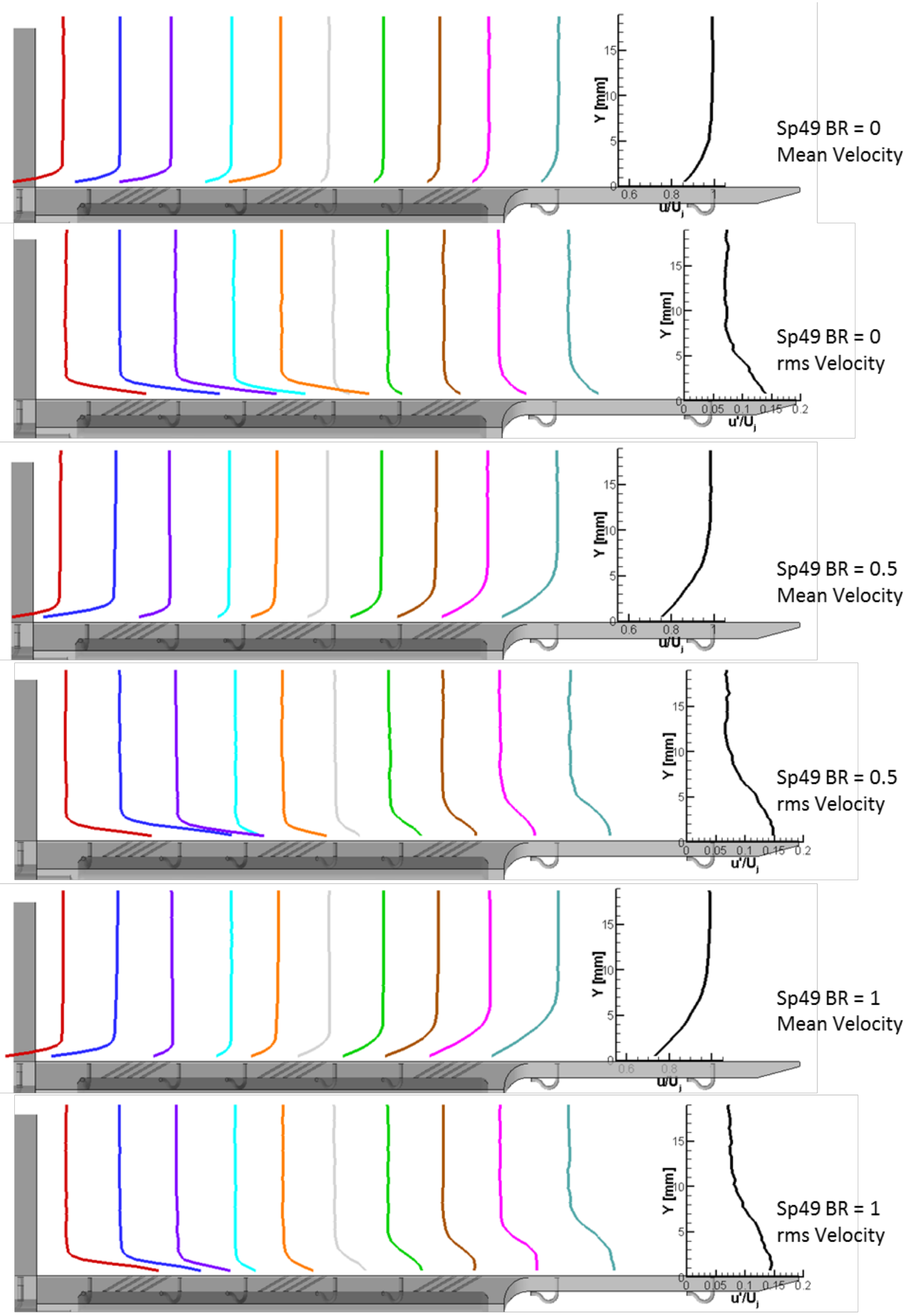

Figure 22. Streamwise PIV mean and rms velocities plotted on the model for Set Point 49, at BR = 0, 0.5, and 1. 

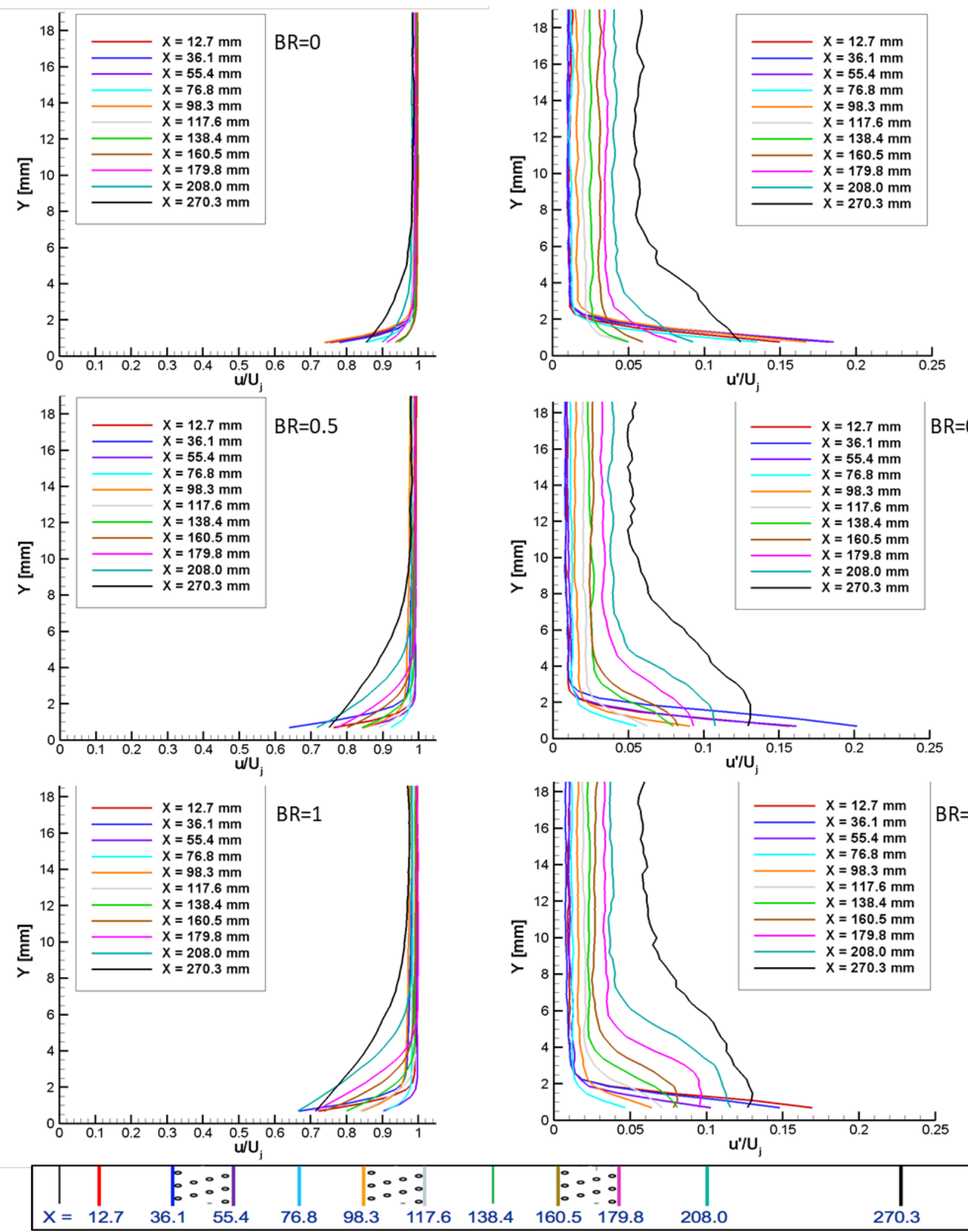

Figure 23. Streamwise PIV mean and rms velocity profiles along the centerline of the plate for Set Point 49 at $\mathrm{BR}=0$, 0.5 and 1 . The color coded key at the bottom shows the measurement stations along the plate where the measurements were collected. 


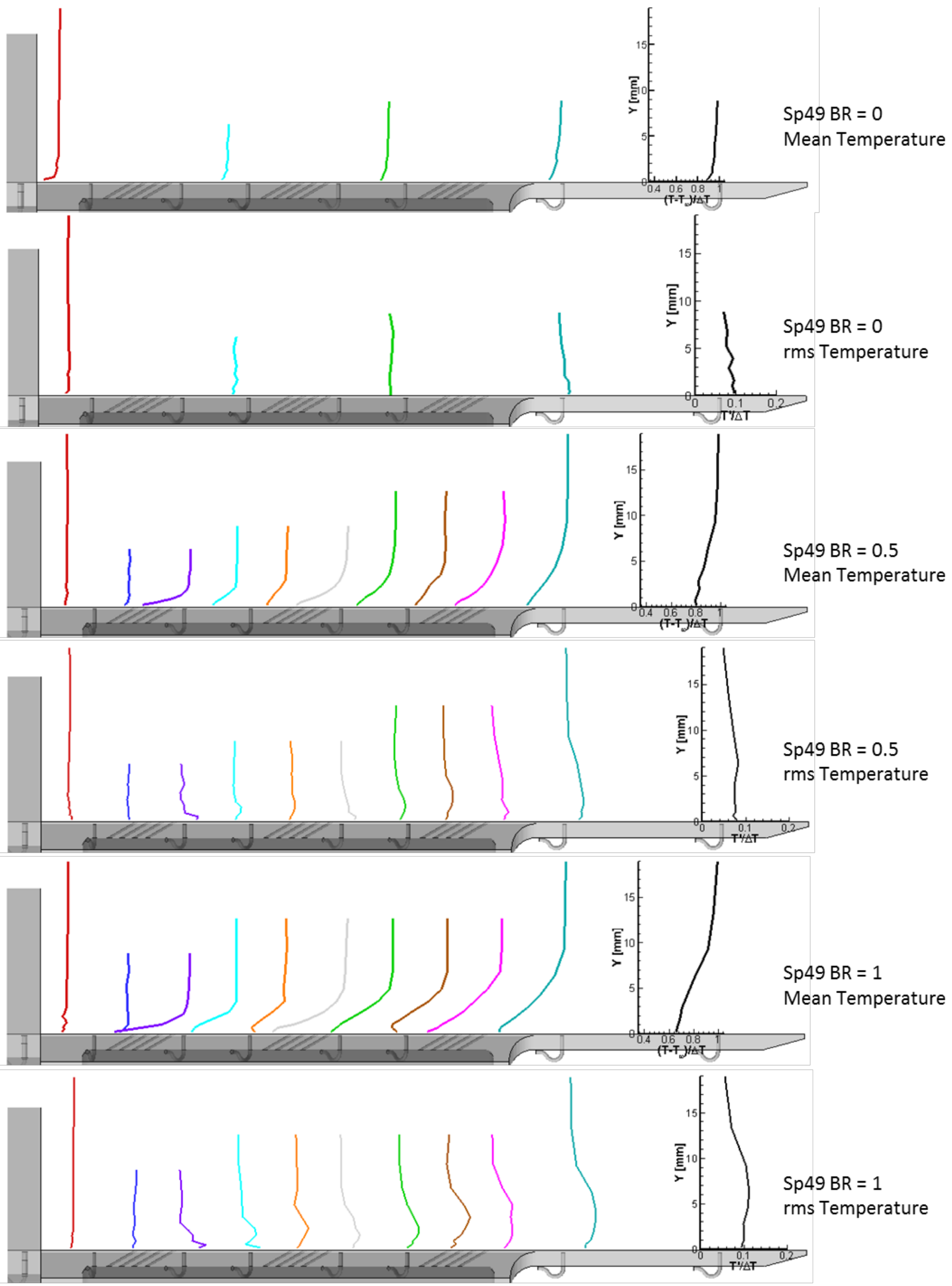

Figure 24. Raman mean and rms temperatures plotted on the model for Set Point 49, at $\mathrm{BR}=0,0.5$, and 1 . 

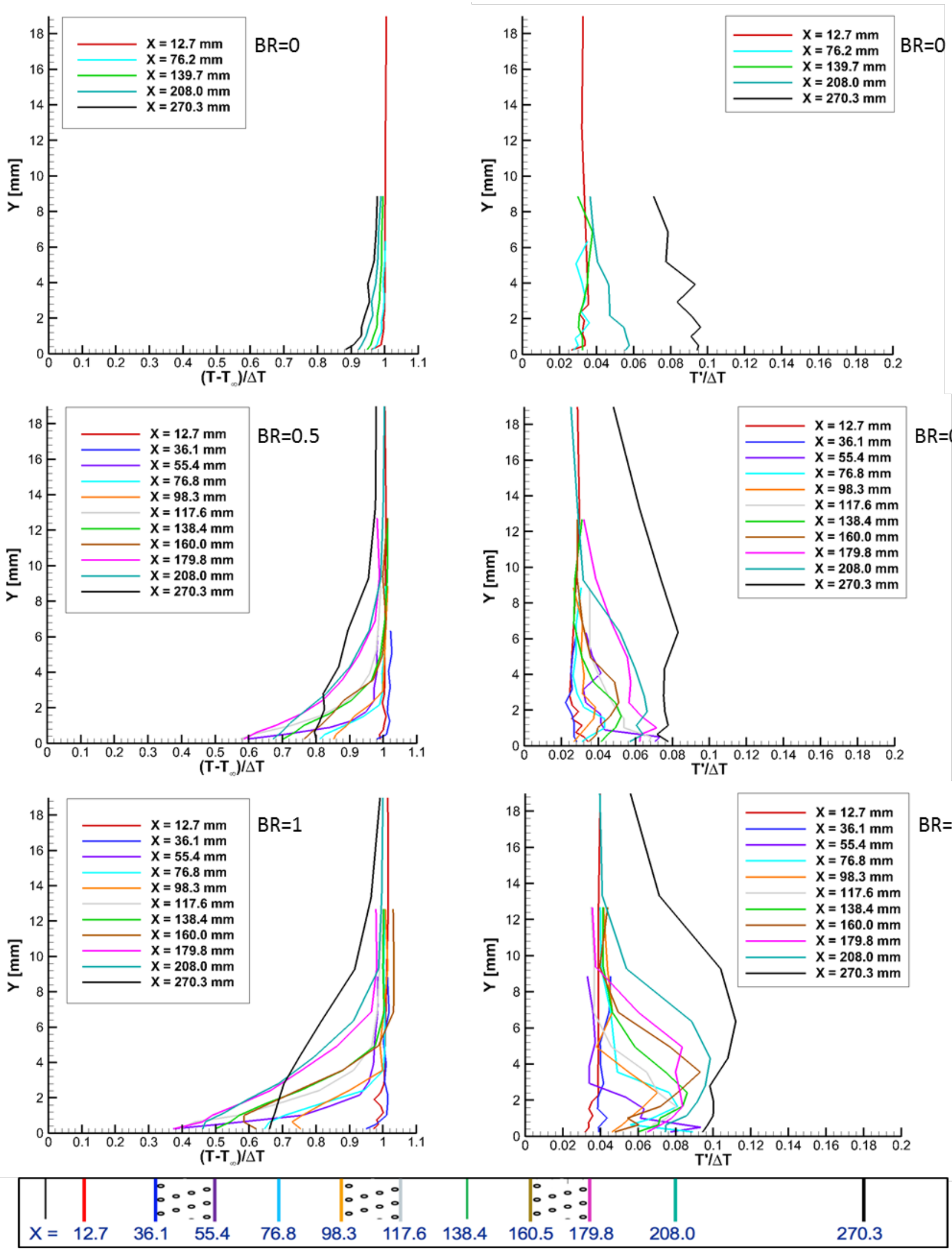

Figure 25. Raman mean and rms temperature profiles along the centerline of the plate for Set Point 49 at $\mathrm{BR}=0,0.5$, and 1. The color coded key at the bottom shows the measurement stations along the plate where the measurements were collected. 


\subsection{IR Camera and Film Effectiveness}

The objectives of the THX Step IV test program were to use multiple diagnostic techniques to characterize the complex film cooling phenomena with the objective of providing benchmark validation data for the evaluation and improvement of current CFD models/techniques. In addition to the off-body measurements, in this section we consider IR thermography. The plate was manufactured from stainless steel and had a highly polished surface in order to provide a good reference surface that could easily be modeled with CFD. The highly polished stainless steel surface became duller as the number of thermal cycles increased, as shown in Fig. 26. The emissivity of dull stainless is nominally $\varepsilon=0.2$, which is on the low end of surface radiation emission and consequently reduces the accuracy of the IR estimates of the plate surface temperature. Painting the surface with a flat black paint would most certainly have raised the emissivity and hence the accuracy of the IR measurements; however, the requirement for having a smooth surface for accurate CFD modeling and avoiding plugging the $1 \mathrm{~mm}$ cooling holes prevented this approach. These image data still provide valuable assessment of the film cooling processes as a function of blowing ratio. IR camera readings were acquired over a more detailed range of blowing ratios than the other diagnostics systems used in the test program.

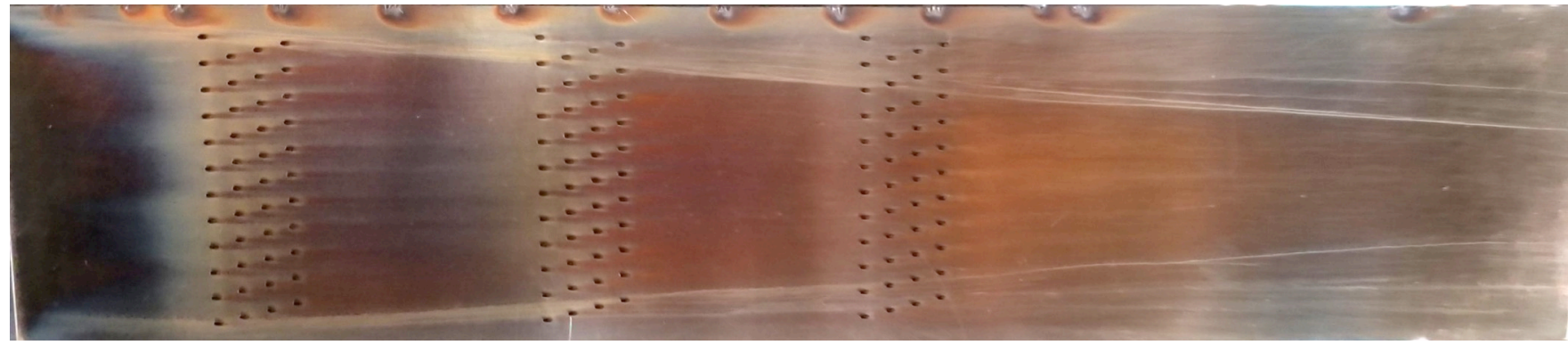

Figure 26. The perforated test plate which has discolored after several thermal cycles. The marks along the bottom edge are from zeroing the Raman laser relative to the model.

The processed IR image data are shown as color contour plots of temperature in Fig. 27 and 28 for Set Point 23 and 49 across a range of blowing ratios. The locations of the thermocouples on the plate surface are also depicted in the plots. Although the air entering from the cooling holes is at much lower temperature than the hot flow above the plate, the higher emissivity of the angled surfaces inside the cooling holes alters the apparent temperature of the holes on the plate, a phenomenon commonly observed in IR thermography [Clausing, 2007]. Therefore, the IR image data inside of the cooling holes is ignored in these color contour plots and is omitted/blanked out in figures 29 through 32 below. In Fig. 27, the IR camera was slightly defocused during the test run, yielding a mildly blurred temperature map. The general surface temperature distribution is still readily apparent. With no cooling, $\mathrm{BR}=0$, the plate is relatively uniform in temperature; however, the cooling plenum ends at $X=178 \mathrm{~mm}$ and the cantilevered section of the plate exhibits lower temperatures due to backside cooling of the plate. At BR $>0$, the temperature on the plate decreases downstream of each cooling hole patch. At $\mathrm{BR}=2$, the lowest temperatures on the plate are observed. The thermocouples marked in solid black squares in both Figs. 27 and 28 were used for the emissivity determination discussed previously in the IR camera equipment installation and setup discussion. A consistent feature in the IR camera maps is the discoloration of the plate surface from the Set Point 49 operating point conditions, as noted above in Fig. 26. At these high temperature Set Points, the plate surface was discolored leaving a distinctive line on the plates at the interface of the hot jet core with the shear layer along the plate surface. The discoloration of the plate surface altered the emissivity of the material and hence are the source of the residual "artifacts" in the IR temperature maps. One last note on the IR images is that there is reflected radiation from inside the nozzle onto the plate surface just at the jet exit yielding artificially high temperature estimates at the exit of the nozzle. 


\section{IR Temperature [K]: $\begin{array}{lllllll}325 & 350 & 375 & 400 & 425 & 450 & 475\end{array}$}
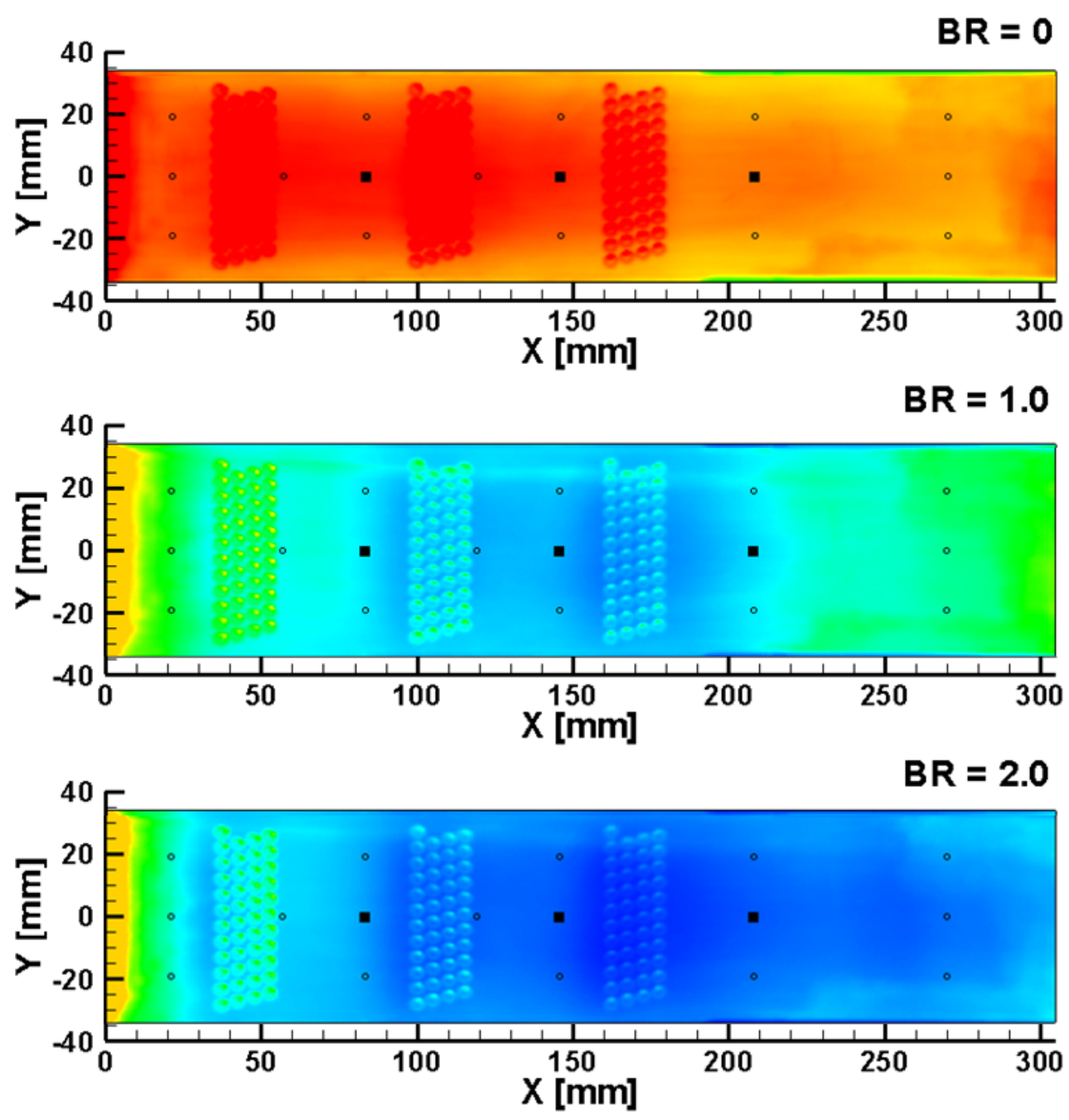

Figure 27. Temperatures measured using an IR camera for Set Point 23 at $\mathrm{BR}=0,1.0$, and 2.0. 

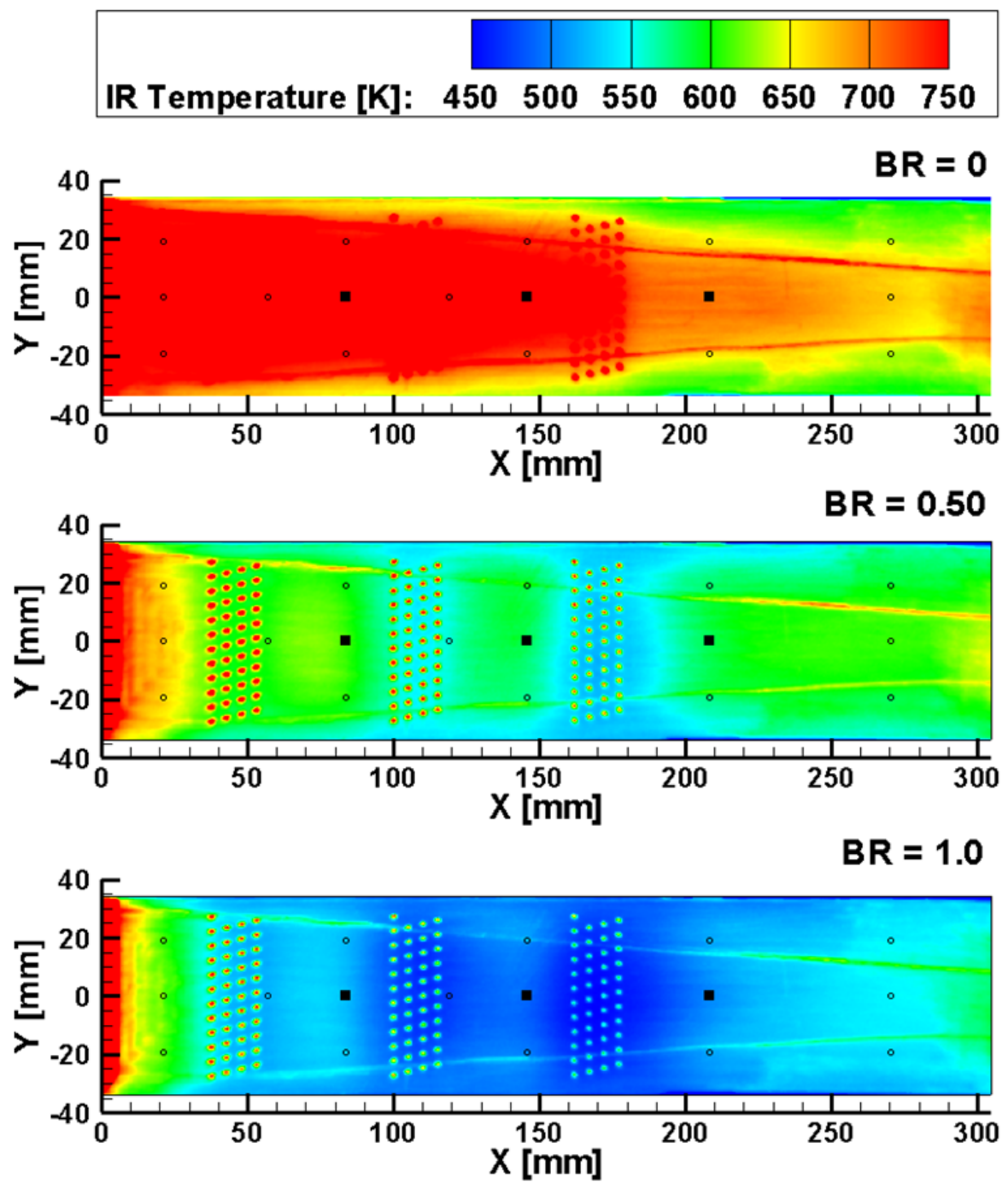

Figure 28. Temperatures measured using an IR camera for Set Point 49 at $\mathrm{BR}=0,0.5$, and 1.0.

In Fig 28, the Set Point 49 data are shown. At $B R=0$, the IR temperature map shows that the plate surface is again relatively uniformly hot within the potential core of the jet up until the last patch of cooling holes. Again, the plate temperature drops at $\mathrm{X}=178 \mathrm{~mm}$ due to backside cooling of the cantilevered plate. For the $\mathrm{BR}=0.5$ case, there is a small amount of cooling happening after the first patch of cooling holes. The cooling becomes more effective after the $2^{\text {nd }}$ and $3^{\text {rd }}$ patches of cooling holes. The plate cooling also appears to be more efficient outside of the jet core as denoted by the large blue region extending out to the edges of the plate. The backpressure generated inside the potential core of the heated jet flow impedes the cooling film over the plate. No direct measurement of this effect was recorded during the test program. For the $\mathrm{BR}=1$ case, the lowest plate temperatures are observed. This result agrees with the Raman temperature measurements where the coolest gas temperature ratios were measured down near the plate surface. The Raman measurements showed that there were some complex interactions occurring within the film cooling layer with/between the cooling patches, the net result from both measurements is that the best plate cooling was at $\mathrm{BR}=1.0$.

The thermocouple measurements along the plate centerline were plotted along with the IR results in Fig. 29 for Set Point 23 at $\mathrm{BR}=0,0.5,1.0,1.5,2.0$ and 2.3. The IR data through the cooling hole patches has been blanked out. The plot shows that the IR data generally match the embedded thermocouple readings. One disparity is at the nozzle exit, likely due to conduction losses from the large mass of metal forming the junction of the nozzle with the thick plenum wall. A second disparity is at the end of the plate, where backside cooling may have impacted the thermocouple readings. The IR data yield a complete mapping of the plate surface temperature, filling in the gaps between the thermocouple readings. The large drop in temperature on the cantilevered section of the plate is observed in the $\mathrm{BR}=0$ line plot. The general trend is lower plate temperatures as blowing ratio is increased. For Set Point 23, the increased plate temperatures after the first patch of cooling holes indicate that the cooling film is not attached to the plate for BR $>1.0$. This result agrees 

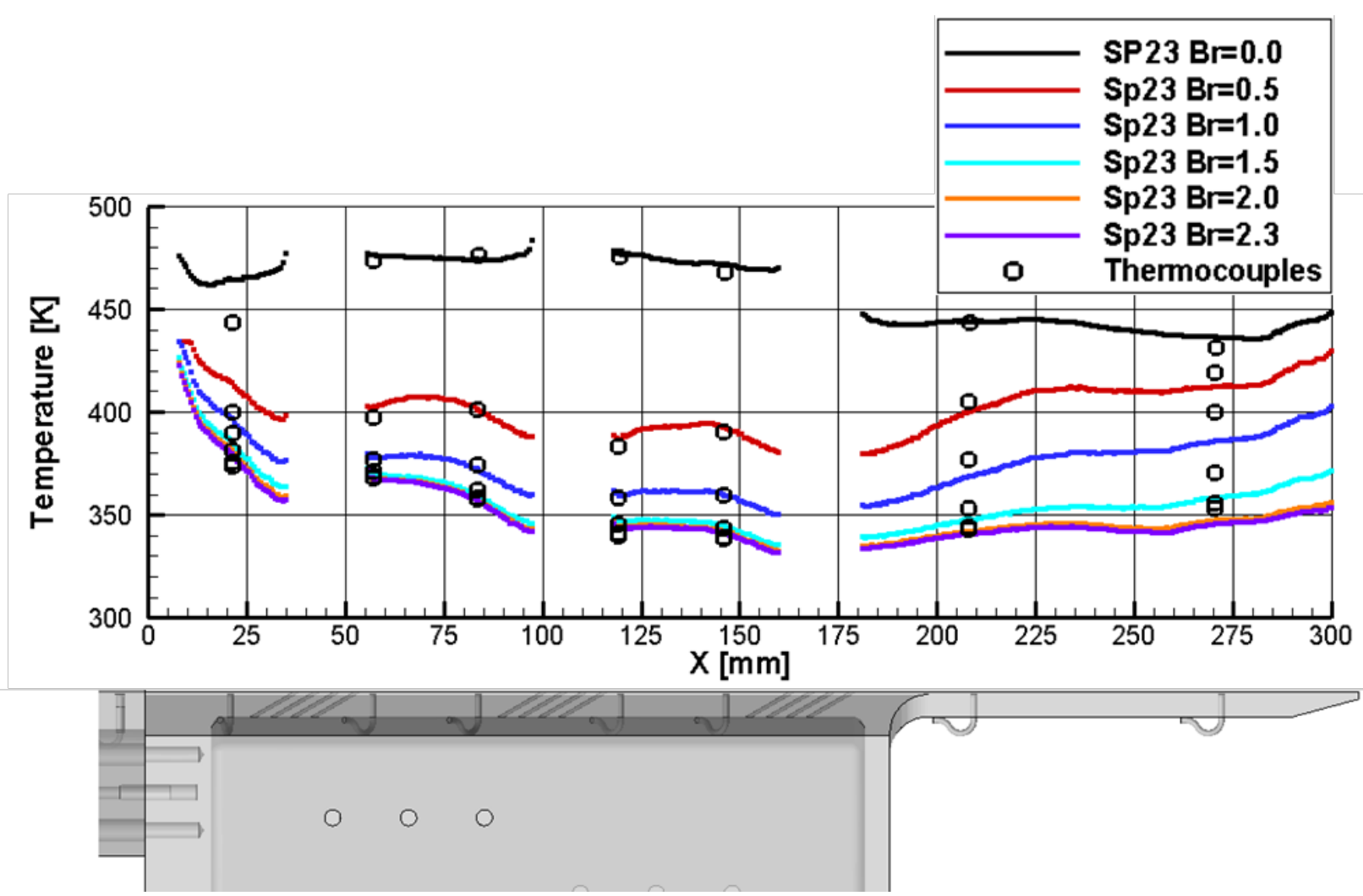

Figure 29. Line plots of temperature down the centerline of the plate for Set Point 23 at BR = 0, 0.5, 1.0, 1.5, 2.0 and 2.3. The region over the injection patch has been blanked out.

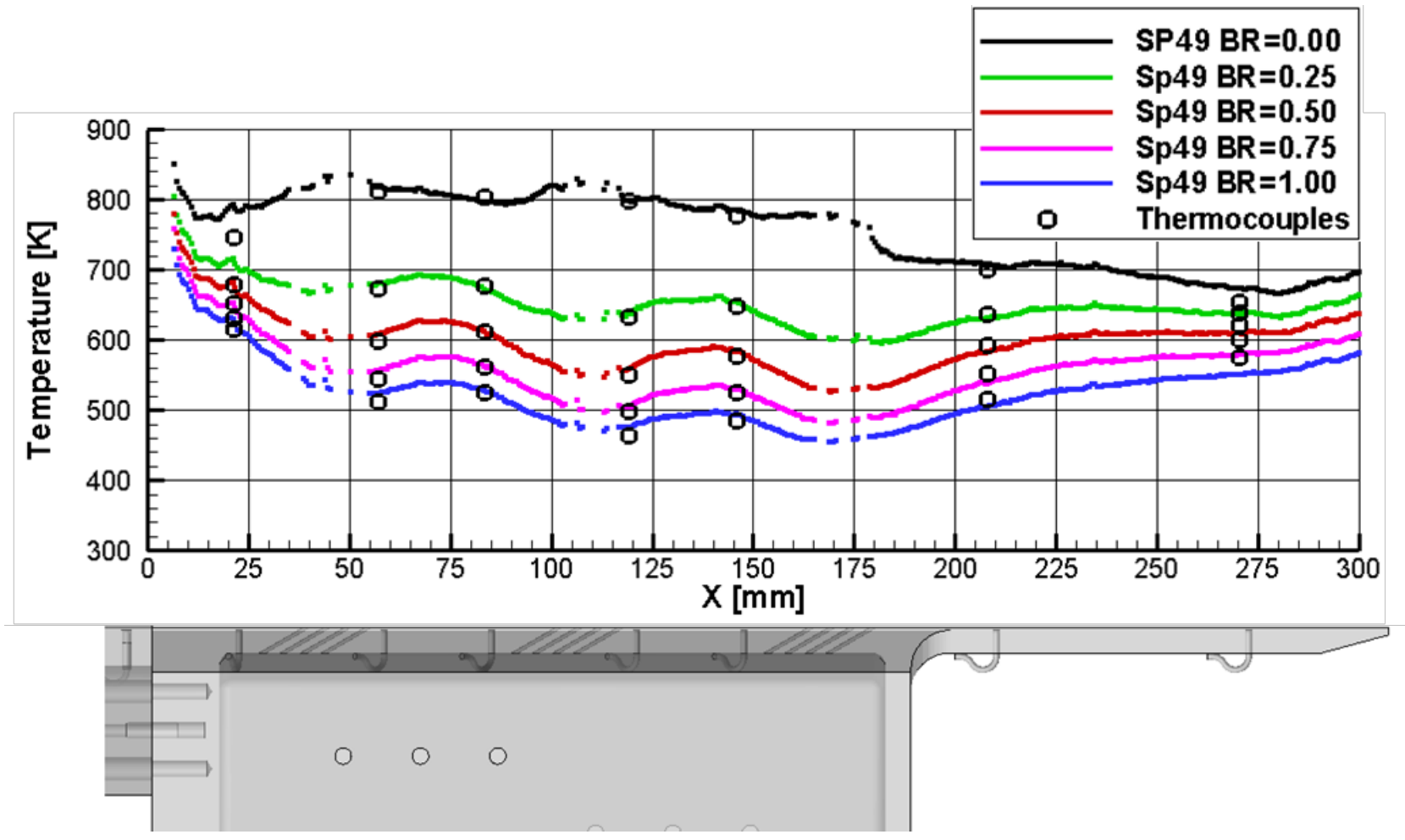

Figure 30. Line plots of temperature down the centerline of the plate for Set Point 49 at $B R=0,0.25,0.5,0.75,1.0$. The region over the injection holes has been blanked out. 
with the Raman temperature profiles at $X=76.8 \mathrm{~mm}$, where the concavity in the normalized temperature profile indicated the cooling film had lifted off of the plate. For all of the BR $>0$ cases, the cantilevered section of the plate increases in temperature from the lowest temperatures achieved after the $3^{\text {rd }}$ patch of cooling holes, compared to the $\mathrm{BR}=0$ case, where the cantilevered section of the plate nominally decreased in temperature.

The thermocouple measurements along the plate centerline are also plotted along with the IR results in Fig. 30 for Set Point 49 at $\mathrm{BR}=0,0.25,0.5,0.75$ and 1.0. The IR data over the cooling holes have again been blanked out. The plot shows that the IR data closely match the embedded thermocouple readings except near the nozzle exit and at the end of the plate as described above for Set Point 23. The large drop in temperature on the cantilevered section of the plate is again observed in the $\mathrm{BR}=0$ line plot. The largest dips in plate temperature are observed within the patches of cooling holes, with a slight rise in plate temperature following each cooling patch. The Raman off-body temperature profiles corroborate the IR readings showing the initial injected cooling film is low temperature, which then quickly increases in temperature as it moves down the plate. It appears that no cooling film lift-off occurred across the limited BR range at Set Point 49. The general trend is lower plate temperatures as blowing ratio is increased.

For $\mathrm{BR}=1.0$, There is a contradiction between the IR measurements and Raman temperature measurements just upstream of the second and third patches. Recall that the Raman mean temperature profiles seem to indicate a very localized blockage and liftoff at these locations with a temperature near the wall slightly higher than a position just slightly further away from the wall. The IR results do not show a locally hotter spot, but it appears that conduction cooling from the air flow through the cooling holes, which are angled through the plate, may dominate the surface temperature upstream of the patch of cooling holes more than the properties/features of the film cooling above the plate.

Next the IR camera data were used to compute the film effectiveness on the plate. Typically in film cooling, an adiabatic wall temperature is used in the definition of film effectiveness. Since the experimental configuration here is not believed to be close to an adiabatic wall situation, we instead use a modified definition for film efficiency using temperature data at $\mathrm{BR}=0$ :

$$
\eta=\frac{T_{B r=0}-T_{B r}}{T_{B r=0}-T_{\text {Plenum }}}
$$

where TPlenum is the temperature of the air inside of the cooling plenum below the test plate and TBR is the IR camera temperature at a given blowing ratio setting. Using $\mathrm{T}_{\mathrm{BR}=0}$ as the reference removes the sensitivity of the experimental hardware to the backside cooling effect noted above. Fig. 31 shows the film effectiveness for Set Point 23, where the cooling efficiency is typically lowest between the patches of cooling holes where the film has dissipated and highest immediately before and after the patches of cooling holes. However, there is a distinct drop in cooling film efficiency across the $1^{\text {st }}$ patch of cooling holes for BR $>1.0$ which again implies lift-off has occurred at the higher blowing ratios. Additionally, for Set Point 23, one can see that additional cooling above BR $=2$ results in minimal increase in effectiveness. Fig. 32 shows the film effectiveness for Set Point 49, where the line plots show a clear and steady trend of increased plate cooling with increasing blowing ratio and the highest film cooling in the vicinity of the patches of cooling holes. For this high temperature ratio case, effectiveness does not seem to increase with addition of more than two patches of cooling holes. Again, effectiveness is lowest in between the patches of cooling holes and highest near the holes. 

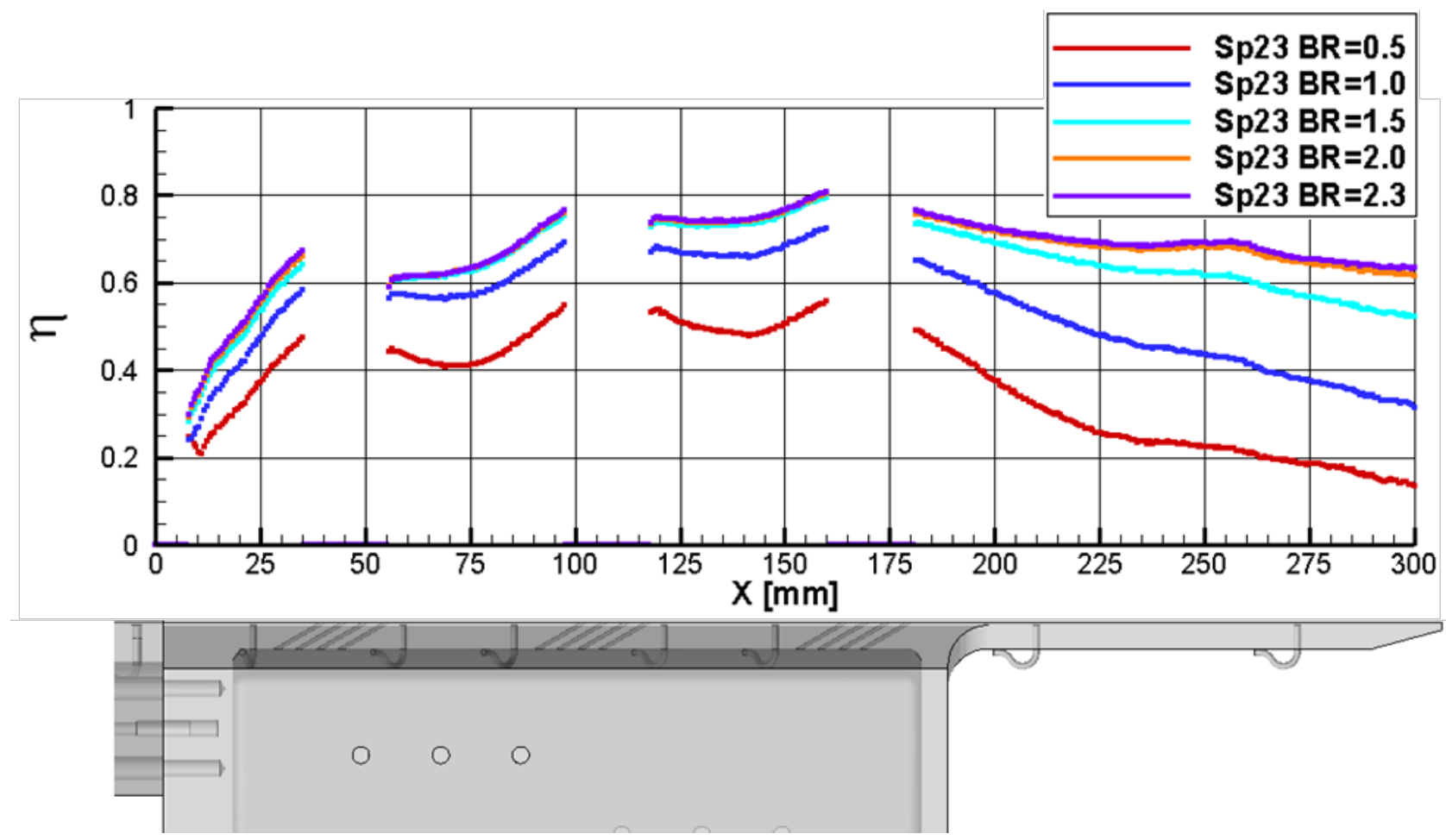

Figure 31. Centerline film effectiveness for Set Point 23 at BR=0.5, 1.0, 1.5, 2.0 and 2.3.

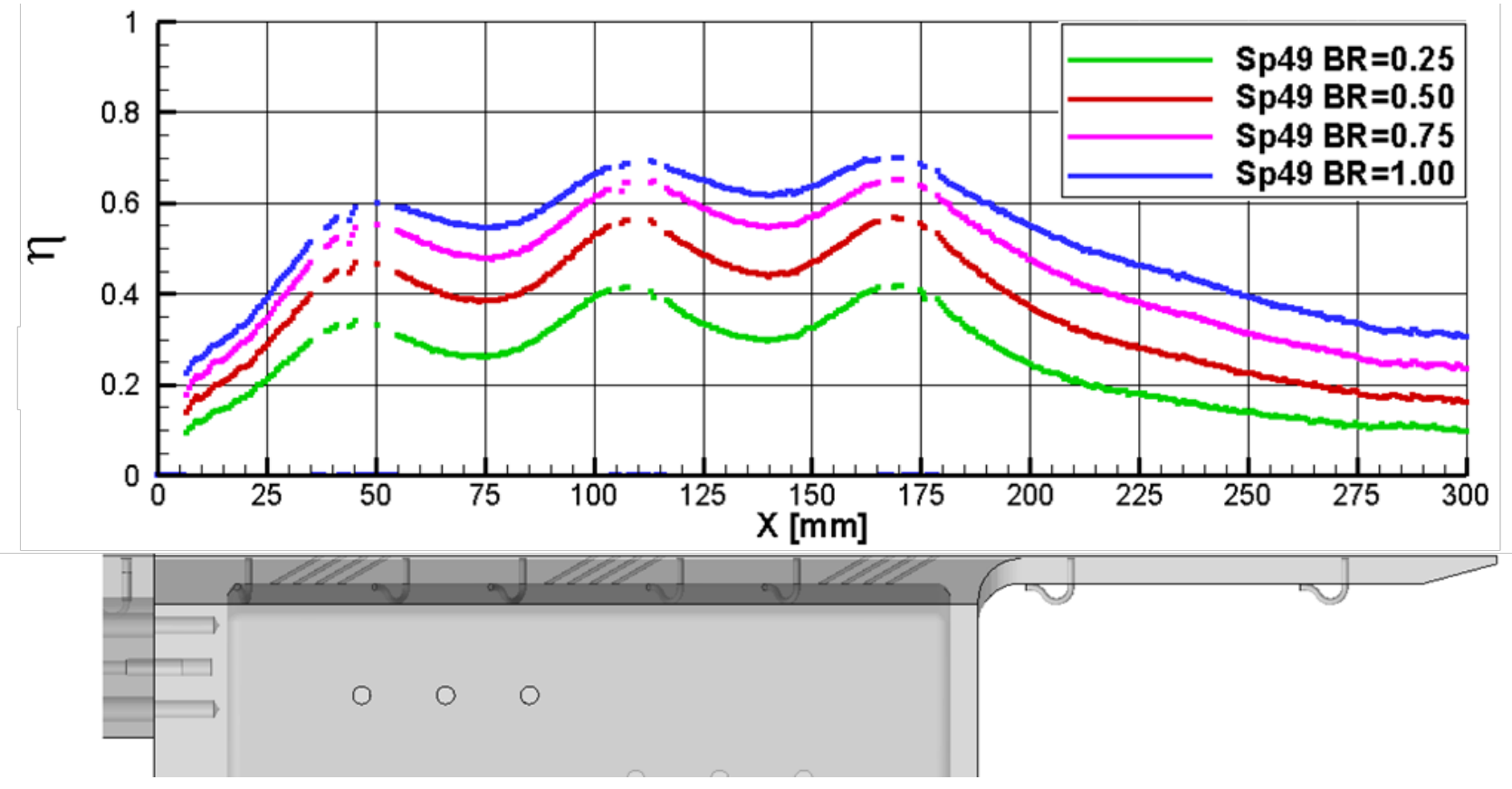

Figure 32. Centerline film effectiveness for Set Point 49 at $\mathrm{BR}=0.25,0.5,0.75,1.0$.

\subsection{Conclusions}

A film cooling experiment was conducted in order to obtain high fidelity turbulent flow data for CFD model evaluation and development. The experiment utilized the heated air from a $68.07 \mathrm{~mm}$ square nozzle blowing over a flat plate equipped with a three patches of 45 cooling holes inclined at $30^{\circ}$ from the plate surface and primary nozzle flow direction. Mach numbers of the heated flow varying from 0.376 to 0.9 , temperature ratios of 1.7 and 2.7, and blowing ratios of 0.0 
to 3.0 were investigated in the test matrix. Rotational Raman temperature measurements were used to acquire mean and rms temperature; PIV measurements along the top of the plate were used to acquire mean and rms velocity measurements and thereby characterize the turbulent aerothermal state. Embedded thermocouples and IR camera measurements were used to measure the surface temperature of the plate. While PIV has been used extensively in recent years for a number of turbulent flow problems in order to obtain mean and rms velocities for turbulence model assessment and improvement efforts, there is a lack of data available in the literature providing mean and rms temperatures. The comprehensive data acquired here is a unique contribution where both velocities and temperatures were measured in a unit flow problem representative of more complex engineering applications in aeropropulsion components. The off-body measurements combined with the on-body surface measurements distinguish the data collected here as the first ever of their breadth and depth.

A correction of the inherent rms variations in the Raman based temperature measurements was applied to the data, yielding normalized rms temperatures in the jet core in agreement with the flow turbulence levels in the jet core. Velocity and turbulence intensity profiles across the shear layers in the injected air were compared with the rms temperature measurements. In most cases the peak fluctuations in velocity and temperature occurred at the same height above the plate. In a few cases the rms temperature peaked higher above the plate than did the peak turbulence across the shear layer. For the case (Set Point 23, BR =2) where the cooling film velocity matched the freestream velocity, there was significantly reduced mean velocity shear, yet thermal mixing between the two co-flowing streams was still observed. This case in particular would be difficult to accurately model using standard RANS techniques where the thermal transport in the energy equation is still typically a function of the mean shear transport as dictated by the Reynolds analogy. In addition to flow field measurements, the surface temperature measurements were used to provide estimates of the film cooling effectiveness. In most cases the off-body temperature measurements corroborated the surface temperature measurements. In a few cases, details of the film cooling above the plate did not agree with the observed surface plate temperatures, when other heat loss mechanisms played a significant role.

Only 2 of the 3 Set Points acquired during the THX Phase IV program were presented in this Part I installment. These data will hopefully provide an enticement for further CFD modeling of the complex flow field studied in this work. The Set Point 42 data set is intended to be used as a Blind Test in a CFD workshop, in 2020. Within the next 2 years the authors plan to publish Part II of this work, where the Set Point 42 data set will be published along with CFD predictions of the flow field measurements.

\section{Acknowledgements}

This work was supported by the Transformative Tools and Technology Project of the Revolutionary Computational Aerosciences (RCA) Program. The Authors would also like to recognize Garrett Clayo for his assistance in the design and fabrication of the optical mounting system, Kathleen Sukel for design of the THX IV plate-plenum system, and K.J. Pederson for structural analysis of this system. The authors would also like to thank the team at the AAPL facility for their assistance in model setup and expertise in operating the SHJAR facility.

\section{References}

1) Bogard, D. G., Thole, K. A., “Gas Turbine Film Cooling," J. of Propulsion and Power, Vol. 21, No. 6, 2006, pp. 122.

2) Smith, S. and M. Mungal, "Mixing, Structure and Scaling of the Jet in Crossflow," J. Fluid Mechanics, Vol. 357, 1998, pp.83-122.

3) Bergeles, G., A. Gosman, and B. Launder, "Near-Field Character of a Jet Discharged through a Wall at $30^{\circ}$ to a Mainstream," AIAA Journal, Vol. 15, No. 4, 1977, pp.499-504.

4) Baldauf, S. M., Schulz, A., and Wittig, S., "High-Resolution Measurements of Local Effectiveness from Discrete Hole Film Cooling," J. of Turbomachinery, Vol. 123, 2001, pp. 758-765.

5) Wernet, M. P., Georgiadis, N. J., Locke, R. J., "PIV and Rotational Raman-Based Temperature Measurements for CFD Validation in a Single Injector Cooling Flow," AIAA Paper 2018-3857 and NASA TM-2018-219739, 2018.

6) Ligrani, P., Goodro, M., Fox, M., Moon, H-K, "Full-Coverage Film Cooling: Film Effectiveness and Heat Transfer Coefficients for Dense Hole Arrays at Different Hole Angles, Contraction Ratios, and Blowing Ratios," J. of Heat Transfer, Vol. 135, 2013, pp.1-14.

7) Ligrani, P., Goodro, M., Fox, M., Moon, H-K, "Full-Coverage Film Cooling: Film Effectiveness and Heat Transfer Coefficients for Dense and Sparse Hole Arrays at Different Blowing Ratios," J. of Turbomachinery, Vol. 134, 2012, pp.1-13. 
8) Lihong, Q., Jingzhou, Z., Xiaoming, T., Minmin, W., "Numerical Investigation On Adiabatic Film Cooling Effectiveness and Heat Transfer Coefficient for Effusion Cooling Over a Transverse Corrugated Surface," Chinese J. of Aeronautics, 2017.

9) Mendez, S., Eldredge, J., Nicoud, F., Poinsotk, T., Shoeybi, M., Iaccarino, G., "Numerical Investigation and Preliminary Modeling of a Turbulent Flow Over a Multi-Perforated Plate," Center for Turbulence Research, Proceedings of the Summer Program, 2006.

10) Andreia, L., Andreinia, A., Bianchinia, C., Caciollia, G., Facchinia, B., Mazzeia, L., Picchia, A., Turrinib, F., "Effusion Cooling Plates for Combustor Liners: Experimental and Numerical Investigations on the Effect of Density Ratio," Energy Procedia, 45, 2014, pp.1402-1411.

11) Mee, D.J., Ireland, P.T., Bather, S., "Measurement of the Temperature Field Downstream of Simulated LeadingEdge Film-Cooling Holes," Exp. in Fluids, Vol 27, No. 3, 1999, pp. 273-283.

12) Andreopoulos, J. and Rodi, W., "Experimental Investigation of Jets in a Crossflow," J. of Fluid Mechanics, Vol. 138, 1984, pp. 93-127.

13) Haldeman, C. W., Mathison, R. M.; Dunn, M. G., Southworth, S., Harral, J. W., Heitland, G., "Aerodynamic and Heat Flux Measurements in a Single Stage Fully Cooled Turbine - Part I: Experimental Approach," ASME Paper GT2006-90966, 2006.

14) Poinsatte, P. E., Heidemann, J. E., Thurman, D. R., "Heat Transfer Measurements for a Film Cooled Turbine Vane Cascade," NASA/TM-2008-215485, GT2008-50651, 2008.

15) Shyam, V., Thurman, D., Poinsatte, P., Ameri, A., Eichele, P., "Long Hole Film Cooling Dataset for CFD Development - Flow and Film Effectiveness," AIAA Paper 2014-3522, July 2014.

16) Yoder, D. A., "Comparison of Turbulent Thermal Diffusivity and Scalar Variance Models," AIAA Paper 2016-1561, Jan. 2016.

17) Bridges, J. and Wernet, M.P., "Measurements of the Aeroacoustic Sound Source in Hot Jets," AIAA Paper 20033130, May 2003.

18) Bridges, J. and Wernet, M. P., "The NASA Subsonic Jet Particle Image Velocimetry (PIV) Dataset," NASA/TM2011-216807, 2011.

19) Panda, J., Seasholtz, R. G., Elam, K. A., "Investigation of Noise Sources in High-Speed Jets Via Correlation Measurements," J. Fluid Mechanics, Vol. 537, 2005, pp. 349-385.

20) Mielke, Amy F., Elam, Kristie A., "Dynamic Measurement of Temperature, Velocity, and Density in Hot Jets Using Rayleigh Scattering," Exp in Fluids, Vol. 47, No. 6, 2009, pp. 673-688.

21) Cutler, A. D., Magnotti, G., Cantu, L., Gallu, E., Rockwell, R. and Goyne, C., "Dual-Pump Coherent Anti-Stokes Raman Spectroscopy Measurements in a Dual-Mode Scramjet," J. Propulsion and Power, Vol. 30, No. 3, May-June 2014, pp. 539-549.

22) Tedder, S., P. Danehy, G. Magnotti, and A. Cutler, "CARS Temperature Measurements in a Combustor-Heated Mach 1.6 Jet," AIAA Paper 2009-0524, Jan. 2009.

23) Kuehner, J. P., Tessier, A. F., Kisoma, A., Flittner, J. G., McErlean, M. R., "Measurements of Mean and Fluctuating Temperature in an Underexpanded Jet Using Electrostrictive Laser-Induced Gratings," Exp. in Fluids, Vol. 48, 2010, pp. 421-430.

24) Ajrouche, H., Vervisch, P., Lo, A., Cessou, A., "1D Single-Shot Thermometry in Flames by Spontaneous Raman Scattering Through a Fast Electro-Optical Shutter," 17th International Symposium on Applications of Laser Techniques to Fluid Mechanics Lisbon, Portugal, 2014.

25) Maté, G. Tejeda, and S. Montero, "Raman Spectroscopy of Supersonic Jets of $\mathrm{CO}_{2}$ : Density, Condensation, and Translational, Rotational, and Vibrational temperatures," J. Chem. Phys., Vol. 108, 1998, pp. 2676.

26) Locke, R. J., Wernet, M. P., Anderson, R. C., "Rotational Raman-Based Temperature Measurements in a HighVelocity Turbulent Jet," Meas. Sci. Technol., Vol. 29, No. 1, 2017, also published as NASA/TM-2017-219504.

27) Yoder, D.A., DeBonis, J.R., and Georgiadis, N.J., "Modeling of Turbulent Free Shear Layer Flows," Computers \& Fluids, Vol. 117, 2015, pp. 212-232.

28) Reynolds, A.J., "Turbulent Flows in Engineering," John Wiley and Sons, New York, 1974.

29) Kays, W.M., "Turbulent Prandtl Number - Where Are We?" ASME Trans., Vol. 116, May 1994.

30) Wernet, M. P., Wroblewski, A. C., Locke, R. J., "A Dual-Plane PIV Study of Turbulent Heat Transfer Flows," NASA/TM-2016-219074, 2016.

31) Borghi, M.R., Thurman, D., Poinsatte, P., and Engblom, W.A., "Numerical and Experimental Examination of Turbulent Mixing of a Heated Jet in Crossflow," AIAA Paper 2018-3867, June 2018.

32) Rumsey, C., Smith, B., and Huang, G., 2010, "Description of a Website Resource for Turbulence Modeling Verification and Validation," AIAA Paper 2010-4742, June 2010.

33) DeBonis, J. R., "Prediction of Turbulent Temperature Fluctuations in Hot Jets," AIAA Journal, Vol. 56, No. 8, Aug. 2018, pp. 3097-3111.

34) Ferraro, J. R., and Nakamoto, K., Introduction to Raman Spectroscopy, Academic Press, San Diego, CA, 1994.

35) Wernet, M. P., "Software for Acquiring Image Data for PIV," NASA Tech Briefs, November, pp. 7, 2003. 
36) Wernet M.P., "Symmetric Phase Only Filtering: A New Paradigm for DPIV Data Processing," Meas. Sci. Technol., Vol. 16, No. 3, 2005, pp. 601-618.

37) Taylor, J.R., An Introduction to Error Analysis, University Science Books, Oxford University Press, Mill Valley, 1982, pp. 142-144.

38) Wernet M. P. and Hadley, J. A., “A High Temperature Seeding Technique for Particle Image Velocimetry," Meas. Sci. Technol., Vol. 27, No. 12, 2016.

39) Melling A., "Tracer Particles and Seeding for Particle Image Velocimetry," Meas. Sci. Technol., Vol. 8, No. 12, 1997, pp. 1406-1416.

40) Wernet, M. P., "Real-Time Background Oriented Schlieren: Catching Up With Knife Edge Schlieren," NASA TM2019-220144, 2019.

41) Clausing, L. Terry, 2007, "Emissivity: Understanding the Difference Between Apparent and Actual Infrared Temperatures," Fluke Application Note, Fluke Education Partnership Program, 2007. 